NASA/TM-2002-211497

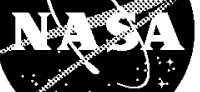

Surface Characterization Techniques: An Overview

Kazuhisa Miyoshi

Glenn Research Center, Cleveland, Ohio

July 2002 
The NASA STI Program Office ... in Profile

Since its founding, NASA has been dedicated to the advancement of aeronautics and space science. The NASA Scientific and Technical Information (STI) Program Office plays a key part in helping NASA maintain this important role.

The NASA STI Program Office is operated by Langley Research Center, the Lead Center for NASA's scientific and technical information. The NASA STI Program Office provides access to the NASA STI Database, the largest collection of aeronautical and space science STI in the world. The Program Office is also NASA's institutional mechanism for disseminating the results of its research and development activities. These results are published by NASA in the NASA STI Report Series, which includes the following report types:

- TECHNICAL PUBLICATION. Reports of completed research or a major significant phase of research that present the results of NASA programs and include extensive data or theoretical analysis. Includes compilations of significant scientific and technical data and information deemed to be of continuing reference value. NASA's counterpart of peerreviewed formal professional papers but has less stringent limitations on manuscript length and extent of graphic presentations.

- TECHNICAL MEMORANDUM. Scientific and technical findings that are preliminary or of specialized interest, e.g., quick release reports, working papers, and bibliographies that contain minimal annotation. Does not contain extensive analysis.

- CONTRACTOR REPORT. Scientific and technical findings by NASA-sponsored contractors and grantees.
- CONFERENCE PUBLICATION. Collected papers from scientific and technical conferences, symposia, seminars, or other meetings sponsored or cosponsored by NASA.

- SPECIAL PUBLICATION. Scientific, technical, or historical information from NASA programs, projects, and missions, often concerned with subjects having substantial public interest.

- TECHNICAL TRANSLATION. Englishlanguage translations of foreign scientific and technical material pertinent to NASA's mission.

Specialized services that complement the STI Program Office's diverse offerings include creating custom thesauri, building customized data bases, organizing and publishing research results ... even providing videos.

For more information about the NASA STI Program Office, see the following:

- Access the NASA STI Program Home Page at http://www.sti.nasa.gov

- E-mail your question via the Internet to help@sti.nasa.gov

- Fax your question to the NASA Access Help Desk at 301-621-0134

- Telephone the NASA Access Help Desk at 301-621-0390

- Write to: NASA Access Help Desk NASA Center for AeroSpace Information 7121 Standard Drive Hanover, MD 21076 
NASA/TM-2002-211497

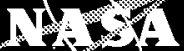

$X+\cdot+$

Surface Characterization Techniques: An Overview

Kazuhisa Miyoshi

Glenn Research Center, Cleveland, Ohio

National Aeronautics and

Space Administration

Glenn Research Center

July 2002 
The Aerospace Propulsion and Power Program at NASA Glenn Research Center sponsored this work.

Available from

NASA Center for Aerospace Information 7121 Standard Drive

Hanover, MD 21076
National Technical Information Service 5285 Port Royal Road Springfield, VA 22100

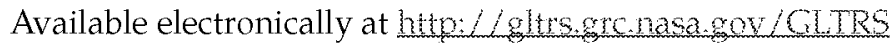




\section{Contents}

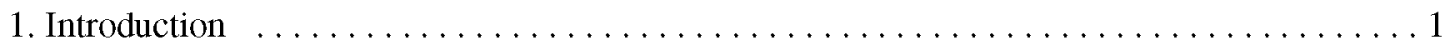

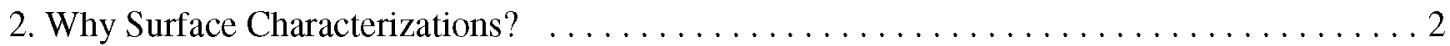

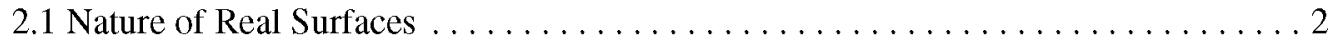

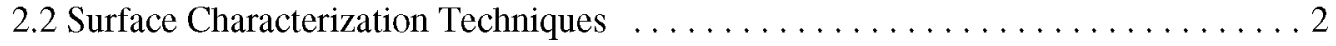

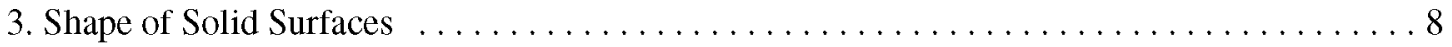

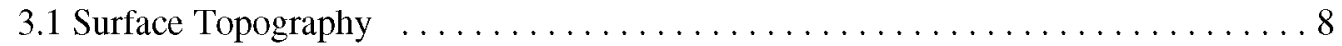

3.2 Surface Metrology Techniques (Surface Profile Probes) $\ldots \ldots \ldots \ldots \ldots \ldots$

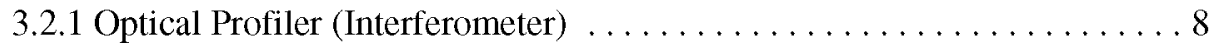

3.2.2 Confocal Microscope . . . . . . . . . . . . . . . . . . . . 10

3.2.3 Scanning Probe Microscopes $\ldots \ldots \ldots \ldots \ldots \ldots \ldots \ldots \ldots \ldots \ldots \ldots$

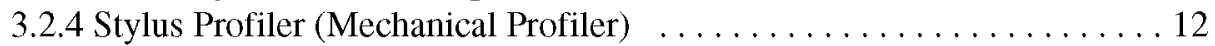

3.2.5 Stereo Microscope . . . . . . . . . . . . . . . . . . . . . . . . 12

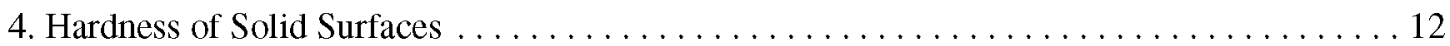

4.1 Hardness as Mechanical Properties Measure . . . . . . . . . . . . . . 12

4.2 Hardness Measurements as Mechanical Properties Probes . . . . . . . . . . . . . . . 13

4.2.1 Nanohardness Measurement . . . . . . . . . . . . . . . . . . . . . 15

4.2.2 Indentation Microhardness Measurement . . . . . . . . . . . . . . . 16

4.2.3 Scratch Microhardness Measurement $\ldots \ldots \ldots \ldots \ldots \ldots \ldots \ldots 22$

5. Elemental Composition and Chemical State of Solid Surfaces $\ldots \ldots \ldots \ldots \ldots \ldots \ldots$

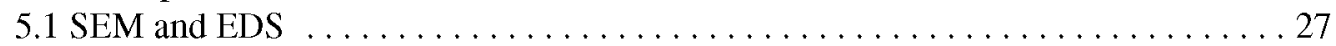

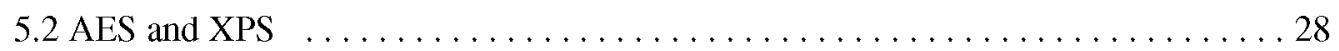

6. Case Study: Characterization of Diamond Films and Coatings . . . . . . . . . . . . 29

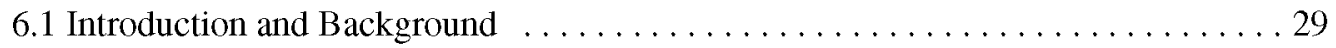

6.2 CVD Diamond Film Deposition Technology ................... 30

6.3 Measurement and Characterization of CVD Diamond $\ldots \ldots \ldots \ldots \ldots \ldots . \ldots . \ldots$.

6.4 Electron Microscopy, Stylus Profilometry, and Atomic Force Microscopy . . . . . 31

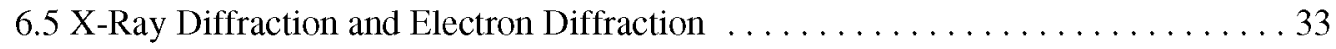

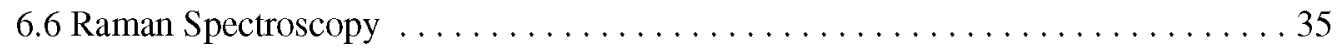

6.7 Rutherford Backscattering Spectroscopy and Elastic Recoil Spectroscopy . . . . . 36

6.8 Friction Measurement . . . . . . . . . . . . . . . . . . . . . 37

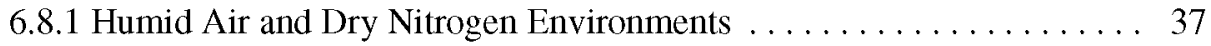

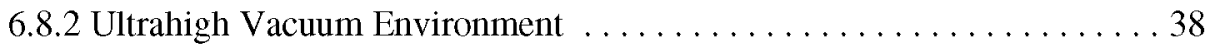

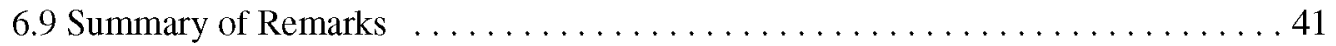

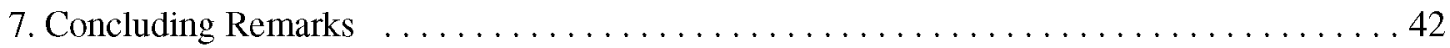

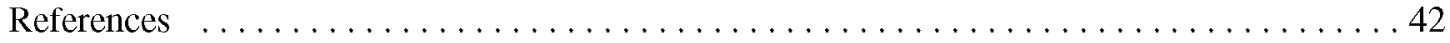




\title{
Surface Characterization Techniques: An Overview
}

\author{
Kazuhisa Miyoshi \\ National Aeronautics and Space Administration \\ Glenn Research Center \\ Cleveland, Ohio 44135
}

To understand the benefits that surface modifications provide, and ultimately to devise better ones, it is necessary to study the physical, mechanical, and chemical changes they cause. This chapter surveys classical and leading-edge developments in surface structure and property characterization methodologies. The primary emphases are on the use of these techniques as they relate to surface modifications, thin films and coatings, and tribological engineering surfaces and on the implications rather than the instrumentation.

\section{Introduction}

As soon as one is confronted with a system where surface properties are involved, one is in trouble. The difficulty with surfaces in most practical situations is that conditions above, at, and below the engineering surface are extremely complex.

The properties of modified engineering surfaces in practical applications can be determined by material and surface analytical techniques (1). Material and surface analyses are evolutionary disciplines. A number of analysis techniques are available for studying modified surfaces, thin films, and coatings from the atomic and electronic levels to macroscopic, engineering-component levels. Such techniques include a variety of physical, chemical, material, and mechanical characterizations. They can provide information that will allow one to select the materials, surface treatments (surface modification techniques and conditions), thin films and coatings, and environments best suited for a particular technical application.

Most materials used in high-technology applications (ranging from high-temperature oxidation, corrosion, thermal insulation, erosion, and wear to hydrophobicity, low adhesion (no stick), and low friction) have a near-surface region with properties differing greatly from those of the bulk material. In general, surface modifications or protective surface coatings (thermal barrier coatings, environmental barrier coatings, and mechanical barrier coatings) are desirable or may even be necessary for a variety of reasons, including unique properties, light weight, engineering and design flexibility, materials conversion, or economics. These objectives can be attained by separating the surface properties from the bulk material properties $(2,3)$.

Surface characterization (diagnostic) techniques are now available for measuring the shape; chemical, physical, and micromechanical properties; composition; and chemical states of any solid surface $(1,4-8)$. Because the surface plays a crucial role in many thermal, chemical, physical, and mechanical processes, such as oxidation, corrosion, adhesion, friction, wear, and erosion, these characterization techniques have established their importance in a number of scientific, industrial, and commercial fields $(1,4-8)$.

This chapter deals with the application of surface characterization techniques to the development of advanced surface modification technology and processes and of tribological coatings and films. These techniques can probe complex surfaces and clarify their interactions in mechanical systems and processes. The primary emphases are on the use of these techniques as they relate to surface modifications, thin films and coatings, and tribological engineering surfaces and on the implications rather than the instrumentation. Finally, a case study describes the methodologies used for surface property measurements and diagnostics of chemical-vapor-deposited diamond films and coatings. 


\section{Why Surface Characterizations?}

\subsection{Nature of Real Surfaces}

A surface, by definition, is an interface, a marked discontinuity from one material to another. Because no change in nature is ever instantaneous, any real surface has a finite depth, and in characterizing a surface one must at some point consider just what this depth is.

Almost all surfaces of bulk substrate materials that are prepared by mechanical techniques contain defects resulting from plastic deformation, fracture, heating, and contamination. Even cleavage faces are rarely defect free. Mechanically abraded, polished, ground, or machined metal, polymer, and ceramic surfaces are extremely rough on an atomic scale, and crystalline structure is distorted. All surfaces contain irregularities, or hills and valleys, which are called surface texture and surface topography. Even in cleaved surfaces of single-crystal materials there are cleavage steps. Surface topography is a permanent record of the deformation and fracture process and provides a materials engineer or scientist with valuable information on the surface properties of materials and coatings.

In addition to the presence of irregularities, commonly called asperities, the solid surface itself is covered with thin contaminant layers of atomic dimensions ( $\sim 2 \mathrm{~nm}$ thick). These contaminant layers are unavoidably present on every surface of any solid matter that has been exposed to air. In other words, the simplest and most common occurrence recognized with real surfaces is that nearly all contain physically or chemically adsorbed material, such as gases, water vapor, and hydrocarbons, that has formed through interaction with the environment. Various hydrocarbons are detected if the component has been near operating machinery because lubricating or processing oils vaporize. Sometimes oxide layers of various depths are present beneath the surface contaminants. Knowledge of the contaminant and oxide layers is of great interest to materials engineers and scientists in surface modifications, thin films and coatings, and tribology as modern technology tries to improve material properties, such as coefficient of friction and resistance against wear, erosion, oxidation, and corrosion in near-surface regions.

Almost every industrial process involves—even depends on-the behavior of a surface. The surface region, which affects a broad spectrum of properties, such as oxidation, corrosion, chemical activity, deformation and fracture, surface energy and tension, adhesion, bonding, friction, lubrication, wear, and contamination, encompasses the first few hundred atomic layers. All surfaces obey the laws of physics and chemistry in their formation, reactions, and combinations. Because any major discontinuity in the solid affects the electronic energy states, effects also arise from surface energy and tension.

The surface structure and chemistry of a thin film, coating, or modified surface region from a bulk substrate material to a surrounding environment include (a) the single-crystal bulk substrate with defects or the polycrystalline bulk substrate with grain boundaries, (b) a deformed (worked) layer, (c) the coating or modified layer, (d) an oxide layer covered by adsorbed contaminants, and (e) the surrounding environmental species. To understand this surface region, it is first necessary to know how the individual parts from (a) to (d) perform in the mechanical, chemical, and physical processes.

\subsection{Surface Characterization Techniques}

Although a wide range of physical and chemical surface analysis techniques is available, certain traits common to many of them can be classified from two viewpoints. Most techniques involve electrons, photons (light), x-rays, neutral species, or ions as a probe beam striking the material to be analyzed. The beam interacts with the material in some way. In some techniques the changes induced by the beam (energy, intensity, and angular distribution) are monitored after the interaction, and analytical information is derived from observing these changes. In other techniques the information used for analysis comes 
from electrons, photons, $\mathrm{x}$-rays, neutral species, or ions that are ejected from the specimen under the stimulation of the probe beam. In many situations several connected processes may be going on more or less simultaneously, with a particular analytical technique picking out only one aspect (e.g., the extent of incident light absorption or the kinetic energy distribution of ejected electrons).

Further, many mechanical techniques are available for assessing the surface roughness and micromechanical properties of material surfaces. Most techniques in this category involve mechanical contacts between a probe and a material surface.

Table 1 briefly summarizes the popular analytical techniques available today for studying the properties and behaviors of solid surfaces:

TABLE 1.-POPULAR ANALYTICAL TECHNIQUES FOR SURFACE, THIN FILM, INTERFACE, AND BULK ANALYSIS OF COATINGS, MODIFIED SURFACE LAYERS, AND MATERIALS

(a) Profilometry and quantitative measurements of film thickness, plastic deformation, and fracture damage

\begin{tabular}{|c|c|c|c|c|c|}
\hline Technique & Main information & $\begin{array}{l}\text { Vertical resolution } \\
\text { (depth probed, } \\
\text { typical) }\end{array}$ & $\begin{array}{c}\text { Lateral resolution } \\
\text { (typical) }\end{array}$ & $\begin{array}{c}\text { Types of } \\
\text { solid } \\
\text { specimen } \\
\text { (typical) } \\
\end{array}$ & $\begin{array}{c}\text { Use } \\
\text { (popularity) }\end{array}$ \\
\hline $\begin{array}{l}\text { Optical } \\
\text { profiler and } \\
\text { laser } \\
\text { interferometry }\end{array}$ & $\begin{array}{l}\text { 3D and 2D imaging } \\
\text { Morphology } \\
\text { Profilometry } \\
\text { Topographic mapping } \\
\text { Film thickness } \\
\text { Wear volume } \\
\text { Scar and crater depth } \\
\text { Defects }\end{array}$ & $-0.1 \mathrm{~nm}$ & $\begin{array}{l}\text { A few sub } \mu \mathrm{m} \text { to } \mathrm{a} \\
\text { few tens of } \mu \mathrm{m}\end{array}$ & All & Medium \\
\hline $\begin{array}{l}\text { Confocal } \\
\text { microscopy }\end{array}$ & $\begin{array}{l}\text { 3D and 2D imaging } \\
\text { Morphology } \\
\text { Profilometry } \\
\text { Topographic imaging } \\
\text { Film thickness } \\
\text { Wear volume } \\
\text { Scar and crater depth } \\
\text { Defects }\end{array}$ & $\begin{array}{l}\text { Variable from a } \\
\text { few nm to a few } \\
\mu \mathrm{m}\end{array}$ & $\begin{array}{l}\text { Optical, } 0.5 \text { to } 4 \mu \mathrm{m} \text {; } \\
\text { SEM, } 1 \text { to } 50 \mu \mathrm{m}\end{array}$ & Almost all & Medium \\
\hline $\begin{array}{l}\text { Optical } \\
\text { scatterometry }\end{array}$ & $\begin{array}{l}\text { Profilometry } \\
\text { Topographic } \\
\text { imaging/mapping } \\
\text { Periodic structure } \\
\text { Morphology } \\
\text { Defects }\end{array}$ & $\geq 0.1 \mathrm{~nm}$ & $\begin{array}{l}\text { A few sub } \mu \mathrm{m} \text { to a } \\
\text { few tens of } \mu \mathrm{m} ; \geq \\
\text { laser wavelength } \lambda / 2 \\
\text { for topography }\end{array}$ & Almost all & $\begin{array}{l}\text { Not } \\
\text { common }\end{array}$ \\
\hline $\begin{array}{l}\text { Light } \\
\text { microscopy } \\
\text { (general) }\end{array}$ & $\begin{array}{l}\text { Imaging } \\
\text { Morphology } \\
\text { Damages } \\
\text { Defects }\end{array}$ & Variable & Variable & All & Extensive \\
\hline $\begin{array}{l}\text { Stylus } \\
\text { profilometry }\end{array}$ & $\begin{array}{l}\text { Profilometry } \\
\text { Topographic tracing } \\
\text { Film thickness } \\
\text { Morphology } \\
\text { Scar and crater depth } \\
\text { Wear volume }\end{array}$ & $0.5 \mathrm{~nm}$ & $100 \mathrm{~nm}$ & $\begin{array}{l}\text { Almost all; } \\
\text { flat smooth } \\
\text { films }\end{array}$ & Extensive \\
\hline $\begin{array}{l}\text { Scanning } \\
\text { tunneling } \\
\text { microscopy } \\
\text { (STM) }\end{array}$ & $\begin{array}{l}\text { Topographic imaging } \\
\text { Compositional } \\
\text { mapping } \\
\text { Morphology } \\
\text { Profilometry } \\
\text { Film thickness } \\
\text { Spectroscopy } \\
\text { Structure } \\
\text { Defects }\end{array}$ & $<0.03$ to $0.05 \mathrm{~nm}$ & Atomic & Conductors & Medium \\
\hline
\end{tabular}

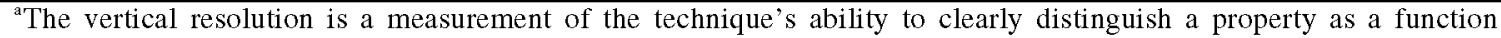
of depth. 
TABLE 1.-CONTINUED.

(a) Concluded.

\begin{tabular}{|c|c|c|c|c|c|}
\hline Technique & Main information & $\begin{array}{c}\text { Vertical } \\
\text { resolution (depth } \\
\text { probed, typical) }\end{array}$ & $\begin{array}{c}\text { Lateral resolution } \\
\text { (typical) }\end{array}$ & $\begin{array}{l}\text { Types of } \\
\text { solid } \\
\text { specimen } \\
\text { (typical) }\end{array}$ & $\begin{array}{c}\text { Use } \\
\text { (popularity) }\end{array}$ \\
\hline $\begin{array}{l}\text { Atomic force } \\
\text { microscopy } \\
\text { (AFM) or } \\
\text { scanning force } \\
\text { microscopy } \\
\text { (SFM) }\end{array}$ & $\begin{array}{l}\text { Topographic imaging } \\
\text { Friction force } \\
\text { mapping } \\
\text { Morphology } \\
\text { Profilometry } \\
\text { Film thickness } \\
\text { Wear volume } \\
\text { Scar and crater } \\
\text { depth } \\
\text { Structure } \\
\text { Defects }\end{array}$ & $<0.03$ to $0.05 \mathrm{~nm}$ & Atomic to $1 \mathrm{~nm}$ & All & Medium \\
\hline $\begin{array}{l}\text { Variable-angle } \\
\text { spectroscopic } \\
\text { ellipsometry } \\
\text { (VASE) }\end{array}$ & $\begin{array}{l}\text { Film thickness } \\
\text { Microstructure } \\
\text { Optical properties }\end{array}$ & Tens of $\mathrm{nm}$ to $\mu \mathrm{m}$ & Millimeter & $\begin{array}{l}\text { Planar } \\
\text { surface } \\
\text { and } \\
\text { interface }\end{array}$ & Medium \\
\hline $\begin{array}{l}\text { X-ray } \\
\text { fluorescence } \\
(\mathrm{XRF})\end{array}$ & $\begin{array}{l}\text { Film thickness ( } 1 \text { to } \\
10^{4} \mathrm{~nm} \text { ) } \\
\text { Element composition } \\
\text { (qualitative } \\
\text { mapping) }\end{array}$ & $10 \mu \mathrm{m}$ & 10 to $150 \mu \mathrm{m}$ & $\begin{array}{l}\text { All but } \\
\text { low-Z } \\
\text { elements: } \\
\mathrm{H}, \mathrm{He} \text {, } \\
\text { and Li }\end{array}$ & Extensive \\
\hline
\end{tabular}

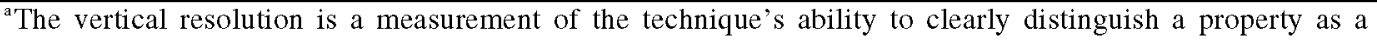
function of depth.

TABLE 1.-CONTINUED.

(b) Surface microprobes for hardness and mechanical strength measurements

\begin{tabular}{|c|c|c|c|c|c|}
\hline Technique & Main information & $\begin{array}{c}\text { Vertical } \\
\text { resolution } \\
\text { (depth probed, }_{\text {typical) }^{\mathrm{a}}}\end{array}$ & $\begin{array}{c}\text { Lateral resolution } \\
\text { (typical) }\end{array}$ & $\begin{array}{l}\text { Types of } \\
\text { solid } \\
\text { specimen } \\
\text { (typical) }\end{array}$ & $\begin{array}{c}\text { Use } \\
\text { (popularity) }\end{array}$ \\
\hline $\begin{array}{l}\text { Mechanical } \\
\text { strength } \\
\text { microprobe } \\
\text { (microhardness } \\
\text { measurements) }\end{array}$ & $\begin{array}{l}\text { Microscale } \\
\quad \text { hardness } \\
\text { Creep deformation } \\
\text { Plastic deformation } \\
\text { Fracture toughness } \\
\text { Strength } \\
\text { Anisotropy }\end{array}$ & $0.3 \mathrm{~nm}$ & $\begin{array}{l}\text { Variable; atomic } \\
\text { to a few tens of } \\
\mu \mathrm{m} \text { using STM, } \\
\text { SFM, AFM, or } \\
\text { optical } \\
\text { microscopy }\end{array}$ & All & Extensive \\
\hline $\begin{array}{l}\text { Mechanical } \\
\text { strength } \\
\text { microprobe } \\
\text { (nanohardness) }\end{array}$ & $\begin{array}{l}\text { Nanoscale hardness } \\
\text { Young's modulus } \\
\text { Creep deformation } \\
\text { Fracture toughness } \\
\text { Strength } \\
\text { Anisotropy }\end{array}$ & $0.3 \mathrm{~nm}$ & $\begin{array}{l}\text { Atomic to } 1 \mathrm{~nm} \\
\text { using STM, SFM, } \\
\text { or AFM }\end{array}$ & All & Medium \\
\hline $\begin{array}{l}\text { Micrometer and } \\
\text { nanometer } \\
\text { scratch } \\
\text { hardness } \\
\text { measurements }\end{array}$ & $\begin{array}{l}\text { Adhesion failure of } \\
\text { thin films and } \\
\text { coatings } \\
\text { Abrasion resistance } \\
\text { Scratch hardness } \\
\text { Deformation } \\
\text { Friction } \\
\text { Fracture } \\
\text { Strength } \\
\text { Anisotropy }\end{array}$ & $0.3 \mathrm{~nm}$ & $\begin{array}{l}\text { Variable; atomic } \\
\text { to a few tens of } \\
\mu \mathrm{m} \text { using STM, } \\
\text { SFM, AFM, or } \\
\text { optical } \\
\text { microscopy }\end{array}$ & All & Medium \\
\hline
\end{tabular}

${ }^{a}$ The vertical resolution is a measurement of the technique's ability to clearly distinguish a property as a function of depth. 
TABLE 1.-CONTINUED.

(c) Elemental composition and chemical state measurements

\begin{tabular}{|c|c|c|c|c|c|}
\hline Technique & Main information & $\begin{array}{c}\text { Vertical } \\
\text { resolution } \\
\text { (depth probed, } \\
\text { typical) } \\
\end{array}$ & $\begin{array}{l}\text { Lateral resolution } \\
\text { (typical) }\end{array}$ & $\begin{array}{l}\text { Types of } \\
\text { solid } \\
\text { specimen } \\
\text { (typical) }\end{array}$ & Use (popularity) \\
\hline $\begin{array}{l}\text { Scanning electron } \\
\text { microscopy (SEM) }\end{array}$ & $\begin{array}{l}\text { Imaging } \\
\text { Morphology } \\
\text { Elemental composition } \\
\text { Damages } \\
\text { Defects } \\
\text { Crystallography } \\
\text { Grain structure } \\
\text { Magnetic domains }\end{array}$ & $\begin{array}{l}\text { Variable from a } \\
\text { few nm to a few } \\
\mu \mathrm{m}\end{array}$ & $\begin{array}{l}1 \text { to } 50 \mathrm{~nm} \text { in } \\
\text { secondary } \\
\text { electron mode }\end{array}$ & $\begin{array}{l}\text { Conductors } \\
\text { and coated } \\
\text { insulators }\end{array}$ & Extensive \\
\hline $\begin{array}{l}\text { Electron probe } \\
\text { X-ray microanalysis } \\
\text { (EPMA) }\end{array}$ & $\begin{array}{l}\text { Elemental composition } \\
\text { SEM imaging } \\
\text { Compositional mapping }\end{array}$ & $1 \mu \mathrm{m}$ & 0.5 to $1 \mu \mathrm{m}$ & All & Medium \\
\hline $\begin{array}{l}\text { Energy-dispersive } \\
\text { x-ray spectroscopy } \\
\text { (EDS) or wavelength- } \\
\text { dispersive x-ray } \\
\text { spectroscopy (WDS) }\end{array}$ & $\begin{array}{l}\text { Elemental composition } \\
\text { ( } Z \geq 5 \text {; boron to } \\
\text { uranium) } \\
\text { Spectroscopy } \\
\text { Imaging and mapping }\end{array}$ & 0.02 to $1 \mu \mathrm{m}$ & $\begin{array}{l}0.5 \text { to } 1 \mu \mathrm{m} \text { for } \\
\text { bulk specimens; } \\
\text { as small as } 1 \mathrm{~nm} \\
\text { for thin } \\
\text { specimens }\end{array}$ & All & Medium \\
\hline $\begin{array}{l}\text { High-resolution } \\
\text { electron energy-loss } \\
\text { spectroscopy (EELS) }\end{array}$ & $\begin{array}{l}\text { Elemental composition } \\
\text { Chemical state } \\
\text { Bonding state } \\
\text { Imaging }\end{array}$ & $2 \mathrm{~nm}$ & $1 \mathrm{~mm}^{2}$ & $\begin{array}{l}\text { Ultra-high- } \\
\text { vacuum- } \\
\text { compatible } \\
\text { solids }\end{array}$ & Not common \\
\hline $\begin{array}{l}\text { Auger electron } \\
\text { spectroscopy (AES) }\end{array}$ & $\begin{array}{l}\text { Elemental composition } \\
\text { (except } \mathrm{H} \text { and } \mathrm{He} \text { ) } \\
\text { Chemical state } \\
\text { Depth profiling } \\
\text { Imaging and mapping }\end{array}$ & 0.5 to $10 \mathrm{~nm}$ & $\begin{array}{l}\text { A few tens of nm } \\
\text { or less }\end{array}$ & $\begin{array}{l}\text { Ultra-high- } \\
\text { vacuum- } \\
\text { compatible } \\
\text { solids }\end{array}$ & Extensive \\
\hline $\begin{array}{l}\text { X-ray photoelectron } \\
\text { spectroscopy (XPS) }\end{array}$ & $\begin{array}{l}\text { Elemental composition } \\
\text { Chemical state } \\
\text { Depth profiling } \\
\text { Imaging and mapping }\end{array}$ & $\begin{array}{l}\text { A few to several } \\
\mathrm{nm}\end{array}$ & $5 \mu \mathrm{m}$ to $5 \mathrm{~mm}$ & $\begin{array}{l}\text { Ultra-high- } \\
\text { vacuum- } \\
\text { compatible } \\
\text { solids }\end{array}$ & Extensive \\
\hline $\begin{array}{l}\text { Fourier transform } \\
\text { infrared spectroscopy } \\
\text { (FTIR) }\end{array}$ & $\begin{array}{l}\text { Chemical species } \\
\text { Stress } \\
\text { Structural inhomogeneity } \\
\text { Defects } \\
\text { Imaging and mapping }\end{array}$ & $10 \mathrm{~nm}$ to $\mu \mathrm{m}$ 's & $20 \mu \mathrm{m}$ to $5 \mathrm{~mm}$ & $\begin{array}{l}\text { All (solid, } \\
\text { liquid, or } \\
\text { gas in all } \\
\text { forms) }\end{array}$ & Extensive \\
\hline Raman spectroscopy & $\begin{array}{l}\text { Identification of } \\
\text { unknown compounds } \\
\text { Chemical state } \\
\text { Bonding state } \\
\text { Structural order } \\
\text { Phase transitions } \\
\text { Imaging and mapping }\end{array}$ & Few $\mu \mathrm{m}$ to $\mathrm{mm}$ & $1 \mu \mathrm{m}$ & $\begin{array}{l}\text { Solids, } \\
\text { liquids, } \\
\text { gases, and } \\
\text { thin films }\end{array}$ & Medium \\
\hline
\end{tabular}

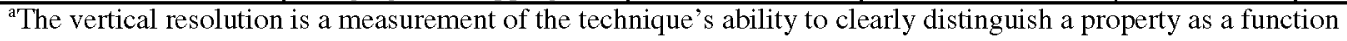
of depth. 
TABLE $1 .-$ CONTINUED.

(c) Concluded.

\begin{tabular}{|c|c|c|c|c|c|}
\hline Technique & Main information & $\begin{array}{c}\text { Vertical } \\
\text { resolution } \\
\text { (depth probed, }^{\text {typical) }}{ }^{\mathrm{a}} \\
\end{array}$ & $\begin{array}{l}\text { Lateral } \\
\text { resolution } \\
\text { (typical) }\end{array}$ & $\begin{array}{l}\text { Types of solid } \\
\text { specimen } \\
\text { (typical) }\end{array}$ & $\begin{array}{c}\text { Use } \\
\text { (popularity) }\end{array}$ \\
\hline $\begin{array}{l}\text { Solid-state } \\
\text { nuclear } \\
\text { magnetic } \\
\text { resonance } \\
\text { (NMR) }\end{array}$ & $\begin{array}{l}\text { Chemical state } \\
\text { Phase identification } \\
\text { Disordered state }\end{array}$ & - & - - & $\begin{array}{l}\text { All; not all } \\
\text { elements }\end{array}$ & Not common \\
\hline $\begin{array}{l}\text { Rutherford } \\
\text { backscattering } \\
\text { spectrometry } \\
\text { (RBS) }\end{array}$ & $\begin{array}{l}\text { Elemental composition } \\
\text { Structure } \\
\text { Defects }\end{array}$ & 2 to $30 \mathrm{~nm}$ & $\begin{array}{l}1 \text { to } 4 \mathrm{~mm} \\
1 \mu \mathrm{m} \text { in } \\
\text { specialized } \\
\text { case }\end{array}$ & $\begin{array}{l}\text { Ultra-high- } \\
\text { vacuum- } \\
\text { compatible } \\
\text { solids }\end{array}$ & Medium \\
\hline $\begin{array}{l}\text { Elastic recoil } \\
\text { spectroscopy } \\
\text { (ERS) }\end{array}$ & $\begin{array}{l}\text { Hydrogen } \\
\text { concentrations in } \\
\text { thin films } \\
\text { Depth profiling }\end{array}$ & $\begin{array}{l}\text { Varies with } \\
\text { depth; } 30 \text { to } \\
60 \mathrm{~nm} \text { at depth } \\
100 \mathrm{~nm} \text { in } \mathrm{Si} \\
\end{array}$ & 1 to $4 \mathrm{~mm}$ & $\begin{array}{l}\text { Ultra-high- } \\
\text { vacuum- } \\
\text { compatible } \\
\text { solids }\end{array}$ & Medium \\
\hline $\begin{array}{l}\text { Secondary ion } \\
\text { mass } \\
\text { spectroscopy } \\
\text { (SIMS) }\end{array}$ & $\begin{array}{l}\text { Chemical state } \\
\text { Imaging } \\
\text { Elemental composition }\end{array}$ & 0.3 to $2 \mathrm{~nm}$ & $10 \mathrm{~nm}$ to $2 \mu \mathrm{m}$ & $\begin{array}{l}\text { All; vacuum- } \\
\text { compatible } \\
\text { solids }\end{array}$ & Extensive \\
\hline $\begin{array}{l}\text { Ion scattering } \\
\text { spectroscopy } \\
\text { (ISS) }\end{array}$ & $\begin{array}{l}\text { Elemental composition } \\
\text { (outermost } \\
\text { monatomic layer) } \\
\text { Imaging (limited) }\end{array}$ & $0.3 \mathrm{~nm}$ & $150 \mu \mathrm{m}$ & $\begin{array}{l}\text { All; vacuum- } \\
\text { compatible } \\
\text { solids }\end{array}$ & Not common \\
\hline
\end{tabular}

aThe vertical resolution is a measurement of the technique's ability to clearly distinguish a property as a function of depth. 
TABLE 1.-CONCLUDED.

(d) Microstructure, crystallography, phase, and defects measurements

\begin{tabular}{|c|c|c|c|c|c|}
\hline Technique & Main information & $\begin{array}{l}\text { Vertical } \\
\text { resolution } \\
\text { (depth }^{\text {probed, }} \\
\text { typical) } \\
\text { ty }\end{array}$ & $\begin{array}{c}\text { Lateral resolution } \\
\text { (typical) }\end{array}$ & $\begin{array}{c}\text { Types of solid } \\
\text { specimen (typical) }\end{array}$ & $\begin{array}{c}\text { Use } \\
\text { (popularity) }\end{array}$ \\
\hline $\begin{array}{l}\text { X-ray diffraction } \\
\text { (XRD) }\end{array}$ & $\begin{array}{l}\text { Crystalline phases } \\
\text { Strain } \\
\text { Crystallite } \\
\text { orientation and size } \\
\text { Atomic arrangements } \\
\text { Defect imaging } \\
\text { Concentration depth } \\
\text { profiling } \\
\text { Film thickness }\end{array}$ & A few $\mu \mathrm{m}$ & $\begin{array}{l}\text { None; } \sim 10 \mu \mathrm{m} \\
\text { with microfocus }\end{array}$ & All & Extensive \\
\hline $\begin{array}{l}\text { Low-energy } \\
\text { electron diffraction } \\
\text { (LEED) }\end{array}$ & $\begin{array}{l}\text { Surface } \\
\text { crystallography and } \\
\text { microstructure } \\
\text { Surface cleanliness } \\
\text { Surface disorder } \\
\text { Imaging }\end{array}$ & $\sim 0.4 \mathrm{~nm}$ & $\begin{array}{l}0.1 \mathrm{~mm}(\sim 10 \mu \mathrm{m} \\
\text { available })\end{array}$ & $\begin{array}{l}\text { Single-crystal } \\
\text { conductors and } \\
\text { semiconductors } \\
\text { Insulators and } \\
\text { polycrystalline } \\
\text { specimens under } \\
\text { special } \\
\text { circumstances }\end{array}$ & Medium \\
\hline $\begin{array}{l}\text { Transmission } \\
\text { electron microscopy } \\
\text { (TEM) }\end{array}$ & $\begin{array}{l}\text { Atomic structure } \\
\text { Microstructure } \\
\text { Crystallographic } \\
\text { structure } \\
\text { Defects } \\
\text { Imaging and mapping } \\
\text { Morphology } \\
\text { Chemical bonding }\end{array}$ & None & $\leq 0.2 \mathrm{~nm}$ & $\begin{array}{l}\text { Conductors, } \\
\text { semiconductors, } \\
\text { and coated } \\
\text { insulators }\end{array}$ & Medium \\
\hline $\begin{array}{l}\text { Reflection high- } \\
\text { energy electron } \\
\text { diffraction } \\
\text { (RHEED) }\end{array}$ & $\begin{array}{l}\text { Surface crystal } \\
\text { structure } \\
\text { 2D and 3D defects }\end{array}$ & 2 to $10 \mathrm{~nm}$ & $200 \mu \mathrm{m} \times 4 \mathrm{~mm}$ & $\begin{array}{l}\text { Single-crystal } \\
\text { conductors and } \\
\text { semiconductors }\end{array}$ & Medium \\
\hline $\begin{array}{l}\text { Cathodolumines- } \\
\text { cence (CL) }\end{array}$ & $\begin{array}{l}\text { Chemical state } \\
\text { Defects }\end{array}$ & $10 \mathrm{~nm}$ to $\mu \mathrm{m}$ & $1 \mu \mathrm{m}$ & All & $\begin{array}{l}\text { Not } \\
\text { common }\end{array}$ \\
\hline $\begin{array}{l}\text { Photoluminescence } \\
\text { (PL), or } \\
\text { fluorescence } \\
\text { spectrometry }\end{array}$ & $\begin{array}{l}\text { Band gaps } \\
\text { Defects } \\
\text { Impurity structure } \\
\text { Chemical state } \\
\text { Imaging and mapping }\end{array}$ & 0.1 to $3 \mu \mathrm{m}$ & 1 to $2 \mu \mathrm{m}$ & All (solid or liquid) & Medium \\
\hline
\end{tabular}

${ }^{a}$ The vertical resolution is a measurement of the technique's ability to clearly distinguish a property as a function of depth.

1. Profilometry and quantitative measurements of film thickness, plastic deformation, and fracture damage

2. Surface microprobes for hardness and mechanical strength measurements

3. Elemental composition and chemical state measurements

4. Microstructure, crystallography, phase, and defect measurements

The table allows quick access to what types of information are provided by these analytical techniques. Also, it provides the typical vertical resolution (or depth probed), typical lateral resolution, typical types of solid specimen, and popularity. The reader will find the basic principles and instrumentation details for a wide range of analytical techniques in the literature (e.g., 4-9). However, the analytical instrumentation field is moving rapidly and within a year current spatial resolutions, sensitivities, imaging and mapping capabilities, accuracies, and instrument costs and sizes are likely to be out of date. Therefore, these references should be viewed with caution. This table should be used as a quick reference guide only. 


\section{Shape of Solid Surfaces}

\subsection{Surface Topography}

The world of the engineer is made of solids whose surfaces acquire their texture (surface roughness, waviness, and lay) from many processes, such as surface modification, coating, thin film deposition, cutting, grinding, lapping, polishing, etching, peening, sawing, casting, molding, and calendering $(10,11)$. Surface topography, or roughness, has a great influence on the surface properties and phenomena of materials, such as surface area, thermal conductivity (or heat contact resistance), electrical conductivity (or electrical contact resistance), bearing area, wear, erosion, corrosion, adhesion, and friction. Surface roughness is an important parameter in characterizing engineering surfaces used in industrial and commercial applications. Surface texture controls the performance of the product.

Profiles of engineering surfaces usually contain three major components: roughness, waviness, and errors of form or lay $(10,11)$. Roughness consists of closely spaced irregularities, the height, width, and direction of which create the predominant surface pattern. Roughness includes those surface features intrinsic to the production process. Waviness encompasses surface irregularities of greater spacing than roughness. Waviness is often the result of heat treatment; machine, workpiece, or specimen deflections; vibrations; or warping strains. Errors of form are gross deviations from the nominal or ideal shape. They are not normally considered part of the surface texture.

Surface roughness occurs at all length scales. The size range of contaminant particles in the environment is enormous. Such particles include human particles (hair, skin flakes), combustion products (smoke, fly ash), and particles produced by abrasion (machining, car tires, sand). In general, any system involving fluid bearings is susceptible to contaminant particle damage. The dimensions of microelectromechanical systems and the flying heights of magnetic hard-disk drives range from tens of micrometers down to a few tens of nanometers. The scale of the world in tribology is essentially determined by the size of the contact areas between surfaces. The diameters of contact areas range from about $100 \mu \mathrm{m}$ down to less than $1 \mathrm{~nm}$, similar to the height range of surface features. The width of that range is large and so is the depth of the worked (deformed) layers.

The unaided eye, fingers, hand lens, optical microscope, stylus profilometer, stereo microscope, optical profiler (interferometer), transmission electron microscope with replicas (sometimes also used in optical microscopy), scanning probe microscopes (e.g., scanning tunneling microscope and atomic force microscope), and scanning electron microscope (Table 1) are typically used for studying surface topography (12). Gross features, such as tool marks, isotropy of the surface texture, surface defects, and discoloration, are often best viewed with the unaided eye. Such viewing is rapid and versatile, allows large areas to be examined, and generally prevents details from obscuring the overall pattern. Also, surface texture and roughness are virtually felt with the fingers as they touch a solid surface. The hand lens (e.g., $10 \mathrm{X}$ ) extends the eye's capability without much loss of speed or surface inspection area. The optical microscope remains one of the most useful and cost-effective tools, in terms of initial cost as well as speed, effectiveness, and versatility of use.

\subsection{Surface Metrology Techniques (Surface Profile Probes)}

3.2.1 Optical Profiler (Interferometer).-The single most useful tool available today to surface engineers and tribologists interested in studying surface damage, erosion, and wear of engineering surfaces is undoubtedly the optical profiler. The optical profiler (noncontact, vertical-scanning, whitelight interferometer and noncontact, vertical-scanning, laser interferometer) can profile an extremely wide range of surface heights and measure surface features without contact (13). It characterizes and quantifies surface roughness, step heights, bearing ratio, height distribution, critical dimensions such as area and volume of damage, eroded craters, and wear scars, and other topographical features. It has 
three-dimensional profiling capability with excellent precision and accuracy; for example, profile heights ranging from $<1 \mathrm{~nm}$ to $5000 \mu \mathrm{m}$ at speeds to $10 \mu \mathrm{m} / \mathrm{s}$ with $0.1-\mathrm{nm}$ height resolution and profile areas as large as 50 by $50 \mathrm{~mm}$ or 100 by $100 \mathrm{~mm}$.

Light reflected from the surface of interest interferes with light from an optically flat reference surface. Deviations in the fringe pattern of bright and dark lines produced by the interference are related to differences in surface height. If an imaging array is used, three-dimensional information can be provided. In general, optical profilers have some advantages-nondestructive measurement, no specimen preparation, and short analysis time under ambient conditions-but also some disadvantages. If the surface is too rough (roughness greater than $1.5 \mathrm{~mm}$ ), the interference fringes can be scattered to the extent that topography cannot be determined. If more than one matrix is involved (e.g., multiple thin films on a substrate), or if the specimen is partially or totally transparent to the wavelength of the measurement system, measurement errors can be introduced. Multiple-matrix specimens can be measured if coated with a layer that is not transparent to the wavelength of light used.

The shape of a surface can be displayed by a computer-generated map developed from digital data derived from a three-dimensional interferogram of the surface. Computer processing and frequency domain analysis result in a quantitative three-dimensional image. Such a map shows details of individual features and also the general topography over an area and describes surfaces. Figure 1 shows optical interferometry images taken from the damaged surface of a typical nickel-base superalloy pin after contact with a gamma titanium aluminide flat (Ti-48Al-2Cr-2Nb in atomic percent) under fretting. Clearly, the surface damage consisted of deposited counterpart material (material transfer), pits, grooves, fretting craters, wear scars, and plastic deformation. Also, the combination of data taken from the optical profilometry, scanning electron microscopy (SEM), and $\mathrm{x}$-ray analysis using energy-dispersive $\mathrm{x}$-ray spectroscopy (EDS) (or wavelength-dispersive $x$-ray spectroscopy (WDS) could be used) verified the presence of $\mathrm{Ti}-48 \mathrm{Al}-2 \mathrm{Cr}-2 \mathrm{Nb}$ on the nickel-base superalloy pin (Fig. 2). The Ti-48Al-2Cr-2Nb failed either in tension or in shear because some of the interfacial adhesive bonds were stronger than the cohesive bonds in the Ti-48Al-2Cr-2Nb. In this fretting wear and fatigue study the failed Ti-48Al-2Cr-2Nb debris subsequently transferred to the nickel-base superalloy surface in amounts ranging from 10 to $60 \%$ of the nickel-base superalloy contact area at all fretting conditions. The thickness of the transferred $\mathrm{Ti}-48 \mathrm{Al}-2 \mathrm{Cr}-2 \mathrm{Nb}$ ranged up to $50 \mu \mathrm{m}$. The quantitative volume and thickness of the transferred material were directly processed by the computer.
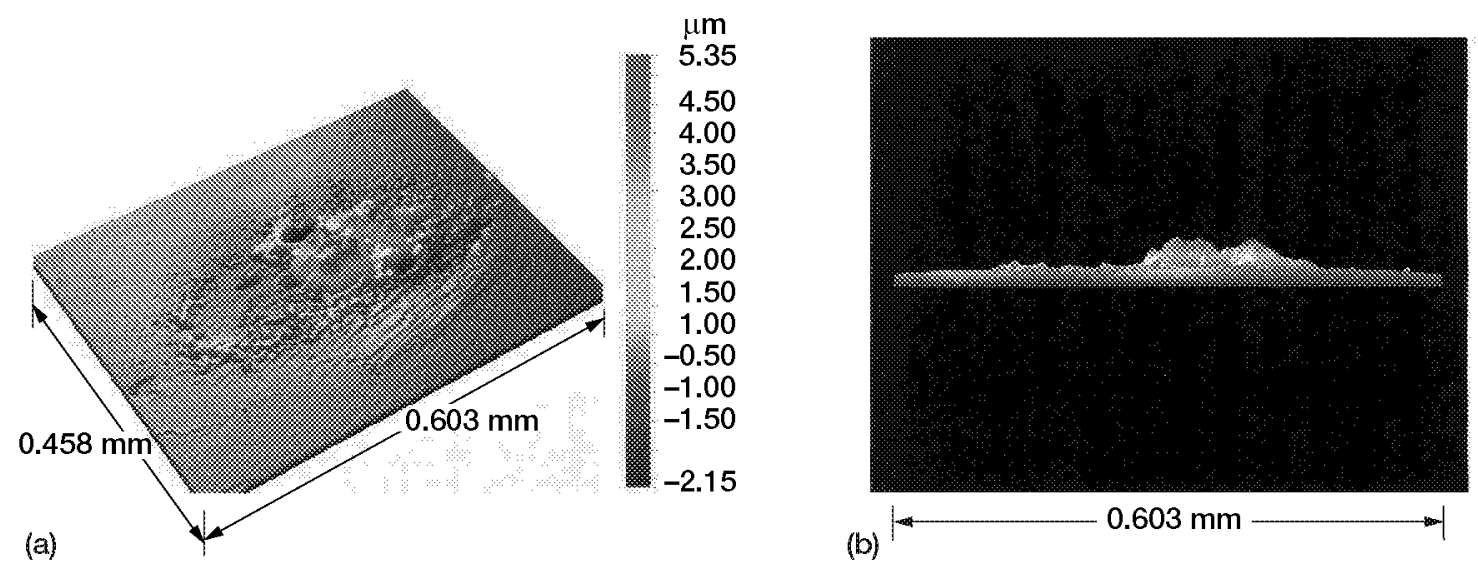

Figure 1.-Optical interferometry images of damaged surface of nickel-base superalloy pin fretted against gamma titanium aluminide flat (Ti-48Al-2Cr-2Nb in atomic percent) in air. Fretting frequency, $50 \mathrm{~Hz}$; slip amplitude, $150 \mu \mathrm{m}$; number of fretting cycles, 1 million; load, $30 \mathrm{~N}$; temperature, $823 \mathrm{~K}$. (a) Threedimensional view. (b) Side view. Volume of material transferred, $1.35 \times 10^{5} \mu \mathrm{m}$. 


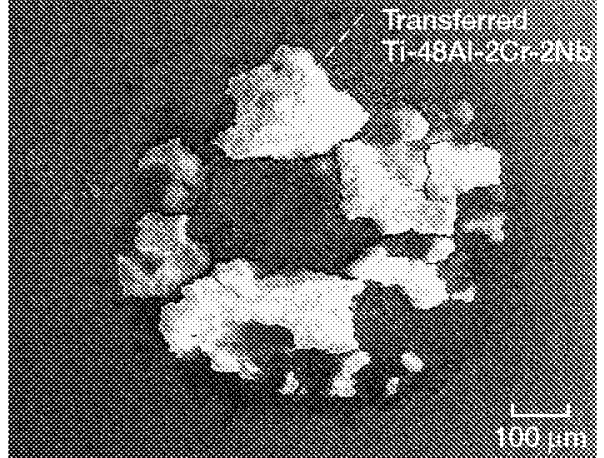

(a)

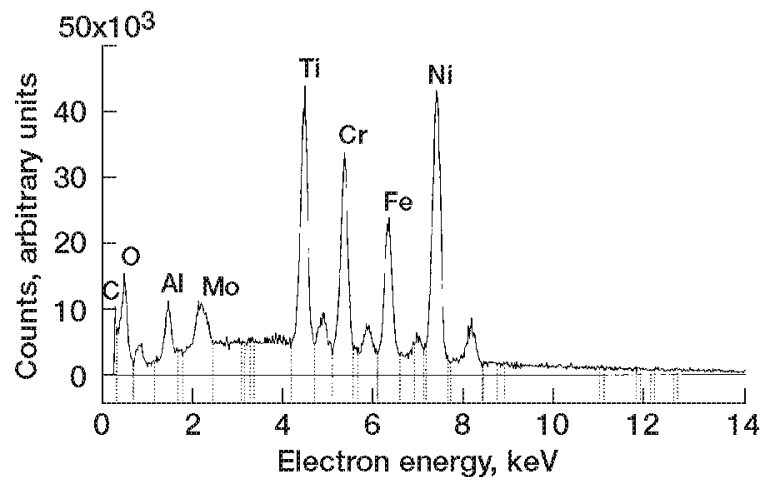

(b)

Figure 2.-SEM backscattered electron image (a) and x-ray energy spectrum (b) of wear scar on nickel-base superalloy pin fretted against Ti-48Al-2Cr-2Nb flat in air at $823 \mathrm{~K}$. Fretting frequency, $80 \mathrm{~Hz}$; slip amplitude, $50 \mu \mathrm{m}$; number of fretting cycles, 1 million; load, $1.5 \mathrm{~N}$; temperature, $823 \mathrm{~K}$.

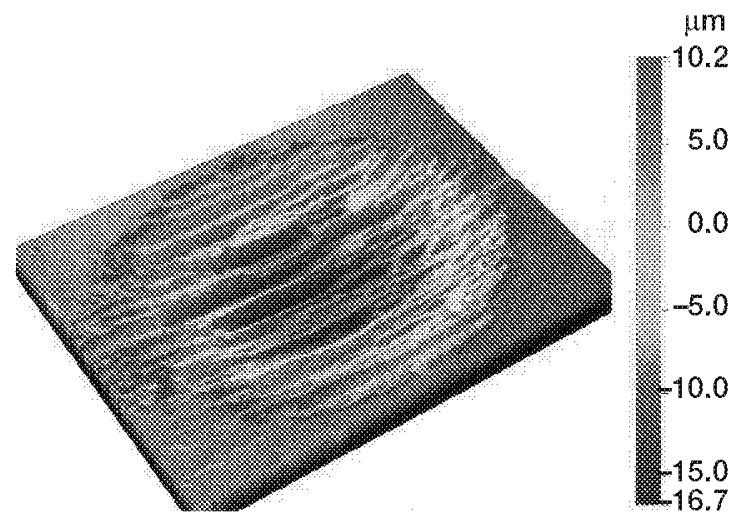

Figure 3.-Optical interferometry three-dimensional image of damaged surface of gamma titanium aluminide ( $\mathrm{Ti}-48 \mathrm{Al}-2 \mathrm{Cr}-2 \mathrm{Nb}$ ) flat fretted against nickel-base superalloy pin in air. Fretting frequency, $50 \mathrm{~Hz}$; slip amplitude, $200 \mu \mathrm{m}$; number of fretting cycles, 1 million; load, $30 \mathrm{~N}$; temperature, $296 \mathrm{~K}$.

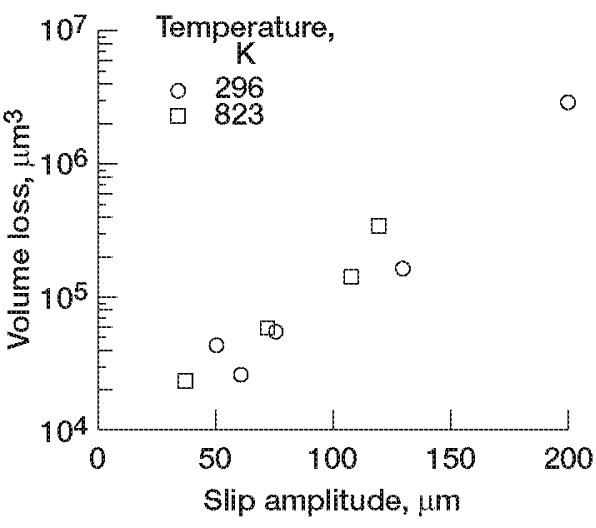

Figure 4.-Wear volume loss as function of sip amplitude, measured by optical interferometry for Ti-48Al-2Cr-2Nb flat fretted against nickel-base superalloy pin in air. Fretting frequency, $50 \mathrm{~Hz}$; number of fretting cycles, 1 million; load, $30 \mathrm{~N}$.

Figure 3 presents a three-dimensional view of the Ti-48Al-2Cr-2Nb wear scar at a slip amplitude of $200 \mu \mathrm{m}$ and a temperature of $296 \mathrm{~K}$. In the wear scar are large, deep grooves where the wear debris particles have scratched the Ti-48Al-2Cr-2Nb surface in the slip direction under fretting. The volume loss of this particular wear scar, calculated from the three-dimensional image, was $4.8 \times 10^{6} \mu \mathrm{m}^{3}$.

Figure 4 shows the volume loss measured by the optical interferometer as a function of slip amplitude for Ti-48Al-2Cr-2Nb in contact with nickel-base superalloy at temperatures of 296 and $823 \mathrm{~K}$. The fretting wear volume generally increased as the slip amplitude increased. An increase in amplitude tends to produce more metallic wear debris, causing severe abrasive wear in the contacting metals, as shown by Fig. 4.

3.2.2 Confocal Microscope.-The popularity of confocal microscopy in characterizing surface damage, such as surface cracks, fracture pits, wear scars and craters, scratches, oxides and debris, and material transfer, arises from its ability to produce blur-free, crisp images of thick specimens at various depths $(7,14)$. This method improves resolution and contrast by eliminating scattered and reflected light from out-of-focus planes. In contrast to a conventional microscope, apertures are used to eliminate all light but 
that from the focused plane on the specimen; a confocal microscope projects only light coming from the focal plane of the lens. Light coming from out-of-focus areas is suppressed. An extended-focus image is obtained by recording the maximum signal at the focal setting, without sacrificing the lateral resolution. Thus, information can be collected from very defined optical sections perpendicular to the microscope axis. Confocal imaging can be performed only with point-wise illumination and detection, which is the most important advantage of using confocal laser scanning microscopy (15). Confocal optics gives a high resolution (e.g., $0.25 \mu \mathrm{m}$ ), far exceeding that of normal light microscopes. The confocal microscope can optically section thick specimens in depth, generating stacks of images from successive focal planes. Subsequently, the stack of images can be used to reconstruct a three-dimensional view of the specimen. The brightness of a pixel depends on the intensity of the light measured from that point in the specimen. Like the optical interferometry system, an image of the whole area of interest is collected either by moving the specimen on computer-controlled scanning stages in a raster scan or moving the beam with scanning mirrors to move the focused spot across the specimen in a raster scan. In either case, the image is assembled pixel by pixel in the computer memory as the scan proceeds. The resolution obtained with the confocal microscope can be a factor of 1.4 better than the resolution obtained with the microscope operated conventionally. By memorizing the stage position at maximum intensity with respect to each scanned pixel, noncontact surface profiling is possible. The measuring range and repeatability for surface profiling can be from 0.1 to $600 \mu \mathrm{m}$ and $0.03 \mu \mathrm{m}$ at $1 \sigma$, respectively.

3.2.3 Scanning Probe Microscopes.-Scanning probe microscopes $(12,16)$ can be considered as derivatives of the stylus profilometer (Table 1). One popular variant is the scanning tunneling microscope (STM). Another is the atomic force microscope (AFM), also called a scanning force microscope (SFM).

Scanning Tunneling Microscope: In this technique a tip is brought to within $1 \mathrm{~nm}$ of the specimen surface, and a small bias voltage of typically 0.01 to $1 \mathrm{~V}$ is applied between them. Under these conditions electrons can penetrate the potential barrier between a specimen and a probe tip, producing an electron tunneling current that varies exponentially with tip-to-surface spacing. If the tip-to-surface spacing increases (or decreases) by $0.1 \mathrm{~nm}$, the tunneling current decreases (or increases) by about a factor of 10 . Therefore, the tunneling current is a sensitive function of tip-to-surface spacing. In most cases, a single atom on the tip will image the single nearest atom on the specimen surface. This tunneling current is the imaging mechanism for the scanning tunneling microscope. A piezoelectric scanner is usually used as an extremely fine positioning stage to move the probe over the specimen (or the specimen under the probe). Clearly, this technique works only with conducting and semiconducting materials. With insulating materials one can add conducting coatings.

Atomic Force Microscope: An atomic force microscope, instead of using the electron tunneling current to measure the tip-to-surface distance, can measure the force of interaction between a specimen surface and a sharp probe tip. The tip, a couple of micrometers long and often less than $10 \mathrm{~nm}$ in diameter, is located at the free end of a cantilever 100 to $200 \mathrm{~mm}$ long. When the tip comes within a few angstroms of the specimen surface, repulsive van der Waals forces between the atoms on the tip and those on the specimen cause the cantilever to deflect, or bend. A detector, such as the position-sensitive photodetector measures the cantilever deflection as the tip is scanned over the specimen or the specimen is scanned under the tip. As a piezoelectric scanner gently traces the tip across the specimen (or the specimen under the tip), the contact force causes the cantilever to bend to accommodate changes in topography. The measured cantilever deflections allow a computer to generate a map of surface topography.

Atomic force microscopes can be used to study insulating and semiconducting materials as well as electrical conducting materials. Most atomic force microscopes currently used detect the position of the cantilever with optical techniques. The position-sensitive photodetector itself can measure light displacements as small as $1 \mathrm{~nm}$. The ratio of the path length between cantilever and detector to the length of the cantilever itself produces a mechanical amplification. As a result, the system can detect even 0.1-nm vertical movements of the cantilever tip. Other methods of detecting cantilever deflection rely on optical interference, a scanning tunneling microscope tip, or piezoresistive detection (fabricating the cantilever from a piezoresistive material). 
The shape of a surface can be displayed by a computer-generated map developed from digital data derived from many closely spaced parallel profiles taken by this process. Such a map shows details of individual features and also the general topography over an area and describes surfaces. Many engineering surfaces have height distributions that are approximately Gaussian (i.e., they can be described by the normal probability function). It is also useful to describe surfaces in terms of the integral of the distribution (bearing ratio), which gives the fraction of the surface at or below each height. The well-known Abbott's bearing curve, which gives the contact area that would exist if the hills were worn down to the given height by an ideally flat body, is the fraction of the surface at or above each height. Many modern surface analyzers provide chart or video displays of height histogram and bearing ratio (Abbott's bearing curve or bearing area curve) as standard features. (See Fig. 24, on page 34.)

3.2.4 Stylus Profiler (Mechanical Profiler).-Today the stylus profiler (contact measurement) is most widely used for measuring surface roughness and analyzing surface topography. The stylusbased metrology system provides long profile measurements and large surface feature measurements. A diamond stylus with a tip radius of a few micrometers moves up and down as it is dragged across a specimen surface. This up-and-down motion effectively replicates the surface topography. Lateral resolution depends on the stylus radius. If the radius of curvature of the surface of interest is smaller than the radius of curvature of the stylus, the measurement will not satisfactorily reproduce the surface. The typical stylus radius is about $3 \mu \mathrm{m}$, but smaller radii down to submicrometer sizes are available. The load applied to the stylus is usually in the millinewton range. In spite of the light loads used, however, the contact pressure is on the order of gigapascals and is sufficiently large to damage surfaces. Sampling distance ranges from tens of micrometers to tens of millimeters. No specimen preparation is required, almost any specimen regardless of engineering material can be measured rapidly, and results can be obtained in seconds. The stylus profiler provides somewhat limited two-dimensional information. For threedimensional topographical information, consecutive line scans are needed. This procedure can be quite time consuming.

3.2.5 Stereo Microscope.-The stereo microscope allows for three-dimensional viewing of specimens, a stereogram, or a pair of stereo pictures taken by an optical or scanning electron microscope. Stereo imaging consists of two images taken at different angles of incidence a few degrees apart. Stereo imaging, in conjunction with computerized frame storage and image processing, can provide threedimensional images with the quality normally ascribed to optical microscopy.

Scanning Electron Microscope: The scanning electron microscope produces micrographs with sufficient resolution to reveal individual features (lateral resolution of 1 to $50 \mathrm{~nm}$ in the secondary electron mode at sampling depths from a few nanometers to a few micrometers, depending on the accelerating voltage and the analysis mode) and yet with a large enough field of view that the interrelation of many such features can be seen. In practice, however, the scanning electron microscope has three disadvantages: specimen size is limited to $10 \mathrm{~cm}$ or smaller, roughness cannot be quantified, and specimens must be vacuum compatible. Cleaving the specimen and taking a cross-sectional view can measure surface features best.

Light Microscope: The light microscope uses the visible or near-visible portion of the electromagnetic spectrum. The technique involves, at its very basic level, the simple, direct visual observation of a specimen with white-light resolution to $0.2 \mu \mathrm{m}$. The surface morphology, color, opacity, and optical properties are often sufficient to characterize and identify a material and its surface.

\section{Hardness of Solid Surfaces}

\subsection{Hardness as Mechanical Properties Measure}

Hardness measurements are a quick, reliable means of quantifying the mechanical properties and performance of modified surfaces, thin films and coatings, and engineering materials. Hardness values 
measured with a specific method represent a scale by themselves, evaluating the mechanical properties and allowing the comparison of materials (17). Hardness measurements can quickly yield quantitative information about the elastic, anelastic, plastic, viscous, and fracture properties of a great variety of both isotropic and anisotropic solids. Hardness measurements can be used to determine the hardness, yield strength, and fracture toughness of a material in a nondestructive manner. The tools used are simple and the specimen sizes needed are typically small, sometimes submicroscopic. It is not necessary to have large specimens to measure strength properties, and it is possible to measure the properties of various microscopic particles within the matrix phase of a polyphase (multiple phase) metal, polymer, mineral, or ceramic material as well as a coated material. Therefore, hardness may be considered to be a strength microprobe (J.J. Gilman's words in (18)) or a mechanical properties microprobe.

\subsection{Hardness Measurements as Mechanical Properties Probes}

The wide variety of hardness measurement methods may be classified as in Table 1(b) and Table 2 $(19,20)$. Many indenters are available for use in hardness measurements. Figure 5 presents the indenters (Vickers, Berkovich, and Rockwell) most commonly used for microhardness and nanohardness measurements. Table 3 briefly describes each commonly used indenter and its uses $(20,21,22)$. The effective included angle of an indenter plays an important role in hardness testing. Some indenters are better than others for specific tasks. Most indenters used in engineering practice are nearly blunt. The indenter, being made of diamond, suffers little deformation during the indentation, and the constraint developed is essentially elastic.
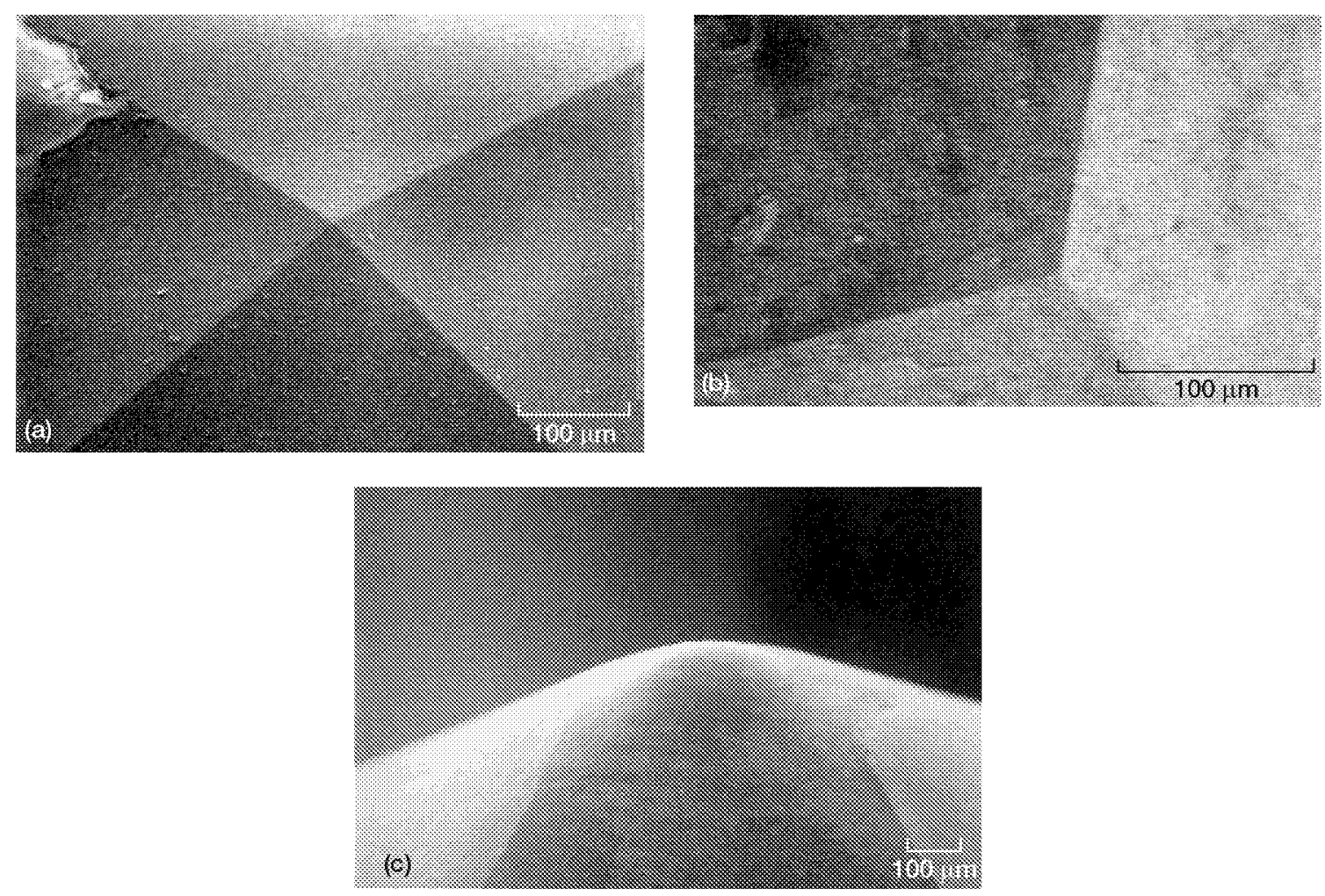

Figure 5.-Commonly used indenters for microhardness and nanohardness measurements. (a) Vickers indenter: four-sided pyramidal tip with included angle of $136^{\circ}$. (b) Berkovich indenter: three-sided pyramidal tip with included angle of $142.3^{\circ}$; average radius of curvature 100 to $200 \mathrm{~nm}$. (c) Spherical indenter showing Rockwell cone diamond with tip radius of $0.2 \mathrm{~mm}$. 
TABLE 2.-HARDNESS MEASUREMENT METHODS

\begin{tabular}{|l|l|}
\hline \multicolumn{1}{|c|}{ Method } & \multicolumn{1}{c|}{ Description } \\
\hline Indentation & $\begin{array}{l}\text { A pyramid, ball, or cone is forced into a surface, and the load per unit area of } \\
\text { impression is taken as the measure of hardness (Vickers, Knoop, Berkovich, } \\
\text { Monotron, Brinell, and Meyer hardnesses). }\end{array}$ \\
\hline Scratch & $\begin{array}{l}\text { It is merely observed whether one material is capable of scratching another (Mohs } \\
\text { and file hardnesses). }\end{array}$ \\
\hline Plowing & $\begin{array}{l}\text { A diamond stylus is moved across a surface under controlled conditions of load and } \\
\text { geometry, and the hardness is determined by the width or depth of the resulting } \\
\text { scratch (Bierbaum, Hankins, and O'Neil hardnesses). }\end{array}$ \\
\hline $\begin{array}{l}\text { Scratch with } \\
\text { physical } \\
\text { characterizations }\end{array}$ & $\begin{array}{l}\text { A diamond stylus is moved across a surface under controlled conditions of load and } \\
\text { geometry, and the hardness, tangential (friction) force, scratch depth, and profile are } \\
\text { determined from the scratch testing. }\end{array}$ \\
\hline Rebound & $\begin{array}{l}\text { An object of standard mass and dimensions is bounced from the specimen surface, and } \\
\text { the height of rebound is taken as the measure of hardness (Shore scleroscope } \\
\text { hardness). }\end{array}$ \\
\hline Damping & $\begin{array}{l}\text { The change in amplitude of a pendulum having a hard pivot resting on the specimen } \\
\text { surface is the measure of hardness (Herbert pendulum). }\end{array}$ \\
\hline Cutting & A sharp tool of given geometry is caused to remove a chip of standard dimensions. \\
\hline Abrasion & $\begin{array}{l}\text { A specimen is loaded against a rotating disk and the rate of wear is taken as a measure } \\
\text { of hardness. }\end{array}$ \\
\hline Erosion & $\begin{array}{l}\text { Sand or abrasive grain is caused to impinge upon the specimen surface under } \\
\text { standard conditions, and loss of material in a given time is taken as the measure of } \\
\text { hardness. }\end{array}$ \\
\hline
\end{tabular}

TABLE 3.-COMMONLY USED INDENTERS FOR HARDNESS MEASUREMENTS

\begin{tabular}{|l|l|}
\hline \multicolumn{1}{|c|}{ Indenter } & \multicolumn{1}{c|}{ Description } \\
\hline Vickers & $\begin{array}{l}\text { A four-sided pyramidal tip with included angle of } 136^{\circ} \text {. In making Vickers hardness } \\
\text { measurements, the lengths of the diagonals of the square indentation are measured. The } \\
\text { Vickers hardness number is independent of the indentation size and therefore of the load. } \\
\text { In this it differs from the Brinell test, but for a given load the Brinell and Vickers } \\
\text { numbers are generally nearly equal, as Table } 4 \text { shows. }\end{array}$ \\
\hline Knoop & $\begin{array}{l}\text { A rhomboidal (four-sided pyramidal) tip with included angles, subtended by the longer } \\
\text { and shorter edges, of } 172.5^{\circ} \text { and } 130^{\circ}, \text { respectively, A parallelogram-shaped indentation is } \\
\text { formed in which the longer diagonal is about seven times the shorter diagonal. In making } \\
\text { Knoop hardness measurements, the longer diagonal is measured and used. The Knoop } \\
\text { indenter often produces satisfactory indentations in microhardness testing of a brittle } \\
\text { material, a material with a directional geometry to its structure, or a thin specimen. }\end{array}$ \\
\hline Berkovich & $\begin{array}{l}\text { A three-sided pyramidal tip with included angle of 142.3 . The Berkovich indenter is the } \\
\text { standard for nanoindentation. Although the indentation formed is triangular, in making } \\
\text { nanohardness measurements, the load and indenter displacement (penetration depth) data } \\
\text { are recorded continuously during loading and unloading to produce a load-displacement } \\
\text { curve from which micromechanical properties, such as hardness and elastic modulus, } \\
\text { can be calculated. }\end{array}$ \\
\hline $\begin{array}{l}\text { A cube corner tip with included angle of } 90^{\circ} \text {. The radius of curvature for a cube corner } \\
\text { tip can be much smaller than that for a Berkovich tip. The sharper tips can work well for } \\
\text { thin films. In Bierbaum scratch hardness tests, the corner of a cube is dragged across the } \\
\text { specimen surface with the leading-edge diagonal inclined at an angle of 35 to the sliding } \\
\text { direction. }\end{array}$ \\
\hline $\begin{array}{l}\text { A conical shaped tip with a spherical end. All these tips are the same except for the } \\
\text { radius of curvature. It is difficult to get the radius of curvature as small as that for the } \\
\text { three- and four-sided pyramidal tips. However, they are good scratching tips because of } \\
\text { the nondirectional geometry at their ends. }\end{array}$ \\
\hline Spherical
\end{tabular}


TABLE 4.-APPROXIMATE HARDNESS CONVERSIONS

\begin{tabular}{|c|c|c|c|c|}
\hline $\begin{array}{l}\text { Vickers } \\
\text { hardness } \\
\text { number }\end{array}$ & $\begin{array}{c}\text { Rockwell C } \\
\text { scale }(1.5-\mathrm{kN} \\
\text { load })\end{array}$ & $\begin{array}{c}\text { Rockwell B scale } \\
\text { (1-kN load; } 1.53-\mathrm{mm} \\
\text { (1/16-in.) diameter ball) }\end{array}$ & $\begin{array}{c}\text { Brinell hardness } \\
\text { number } \\
(30 \text { 000-N load; } 10-\mathrm{mm}- \\
\text { diameter ball })\end{array}$ & $\begin{array}{c}\text { Shore } \\
\text { scleroscope } \\
\text { hardness number }\end{array}$ \\
\hline 900 & 67.0 & -- & -- & 95 \\
\hline 800 & 64.0 & ב-ב-ב- & $\ldots$ & 88 \\
\hline 700 & 60.1 & _._- & $\ldots$ & 81 \\
\hline 600 & 55.2 & ב-ב- & 564 & 74 \\
\hline 500 & 49.1 & -..- & 471 & 66 \\
\hline 400 & 40.8 & -_-- & 370 & 55 \\
\hline 300 & 29.8 & -ב-- & 284 & 42 \\
\hline 200 & - & 91.5 & 190 & 29 \\
\hline 100 & ----- & 56.2 & 95 & - \\
\hline
\end{tabular}

Table 4 compares the various scales of hardness measurement, for the convenience of conversion from one scale to another. The values given here are approximate and only small examples. The greater part of the data can be found in the literature (20-24). Commercial and industrial materials are often quoted in Brinell or Rockwell hardness values. However, the scientific community tends to work mainly with Vickers (or sometimes Knoop) microhardness and with nanohardness using a Berkovich, Vickers, or conical indenter.

For a Brinell test using a ball of diameter $D$ it is customary to use indentation diameters of $0.25 D$ to $0.5 D(20)$. The Brinell hardness test generally uses a 10 -mm-diameter ball that is pressed into the specimen surface under a load of $30 \mathrm{kN}$. The Brinell hardness is inferred by comparing the load and the dimensions of the indentation. A Rockwell test infers the hardness from the depth of penetration and thus enables a direct reading of hardness to be obtained from the instrument. Hard materials are measured on the Rockwell $\mathrm{C}$ scale by using a diamond, rounded-tip cone indenter and a load of $1.5 \mathrm{kN}$. Softer materials are measured on the Rockwell B scale by using a steel ball of approximately $1.5-\mathrm{mm}$ diameter and a load of $1 \mathrm{kN}$.

In the Vickers hardness test a pyramidal-shaped indenter is pressed into the surface, the lengths of the diagonals of indentation are measured, and then the hardness is inferred from the load and the dimensions of the indentation (20). The loads usually are from $10 \mathrm{~N}$ to $1.2 \mathrm{kN}$ for macrohardness measurements and up to $10 \mathrm{~N}$ for microhardness measurements.

4.2.1 Nanohardness Measurement.-Nanohardness measurement, such as by a mechanical properties nano-probe, is today most ideal for thin coatings, surface-modified materials, multiple-phase materials, and composites on almost any type of material: hard, soft, brittle, or ductile. Hardness, Young's modulus, and time-dependent indentation creep can be determined at penetration depths as small as a few tens of nanometers.

An indenter tip, normal to the specimen surface, with a known geometry (e.g., Berkovich or Vickers diamond indenter) is driven into the specimen by applying an increasing load up to some preset value. The load is then gradually decreased until partial or complete relaxation of the specimen has occurred. The load and displacement are recorded continuously throughout this process to produce a load-displacement curve from which the micromechanical properties can be calculated. The applied load and penetration depth data can be analyzed to provide the hardness and elastic modulus of the specimen. Figure 6, for example, presents the load-displacement data taken for a

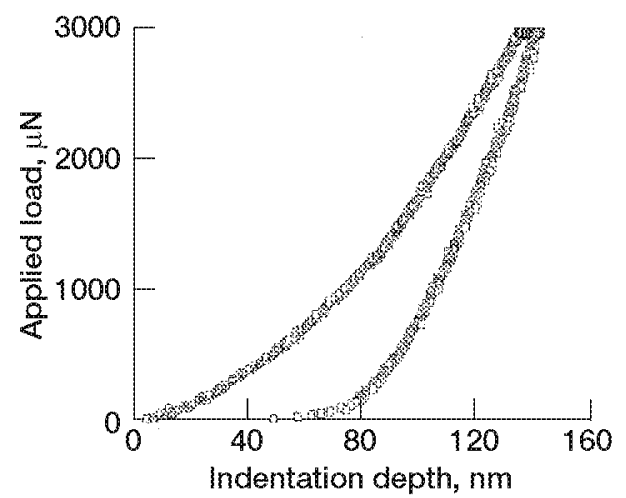

Figure 6.-Load-displacement data (nanohardness measurements) for tungsten carbide/carbon multiple-layered coating deposited on steel. From (21). 


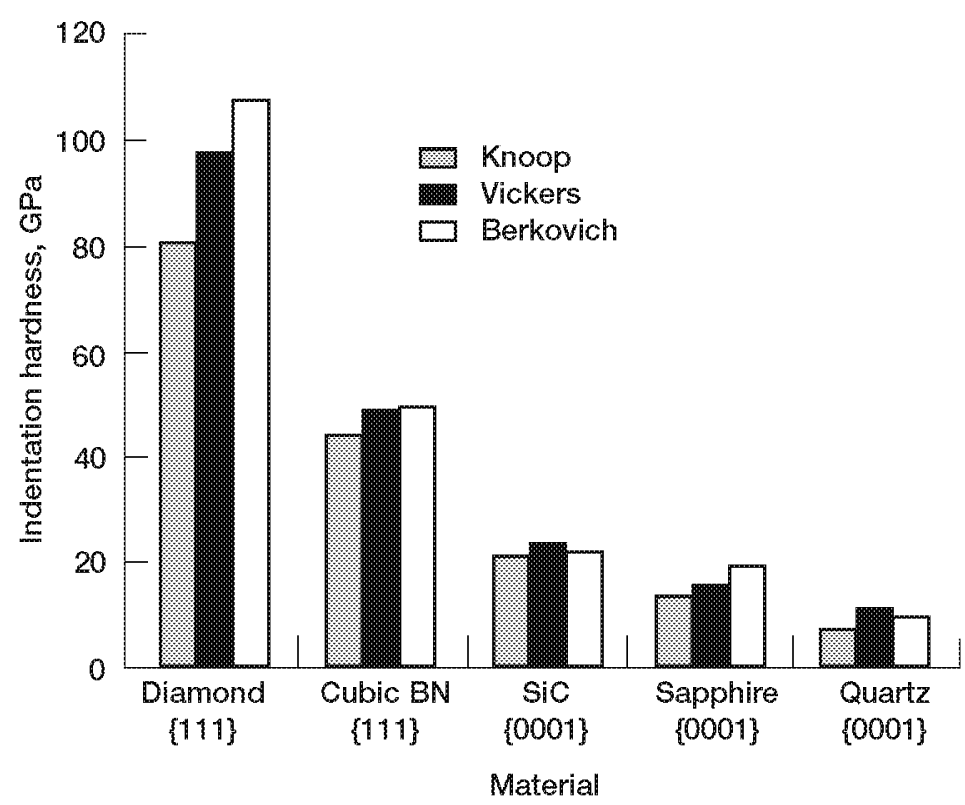

Figure 7.- Indentation hardnesses for diamond and other solid materials. From (25).

tungsten carbide and carbon multiple-layer coating deposited on steel substrates evaluated by nanoindentation (21). The measured hardness, elastic modulus, and maximum contact depth obtained from 25 indentations of the WC/C multiple-layer coating were $11.5 \pm 1.2 \mathrm{GPa}, 113.2 \pm 6.9 \mathrm{GPa}$, and $102.9 \pm$ $6.8 \mathrm{~nm}$, respectively (21).

The combination of a quantitative depth-sensing nano-indenter with scanning probe microscopy can provide nanometer-scale images of indentation, revealing the imprint of the indents and other surface features with nanometer resolution.

4.2.2 Indentation Microhardness Measurement.-Pyramidal indenters (used by Vickers, Knoop, and Berkovich) produce square, rhombohedral, and triangular indentations, respectively, that are plastically deformed. Indentation microhardness measures the plastic strength of the material (i.e., the amount of plastic deformation produced). All the pyramidal indenters have a further advantage in that they yield values, in terms of units of pressure, that can be compared directly with other mechanical properties, such as yield stress, yield strength, and Young's modulus, as described in the previous section.

It has already been established that the hardness measured for a crystalline solid is very much dependent on the indenter shape, normal load, temperature, crystallographic orientation of the material with respect to the indented plane, and impurities. Figure 7 (25), for example, presents the median indentation hardnesses for diamond, cubic boron nitride, silicon carbide, sapphire, and quartz measured with Knoop, Vickers, and Berkovich indenters. For a given crystal the Knoop hardness values are generally lowest, and the Vickers and Berkovich indenters give similar results (25).

Spherical indenters develop tensile stresses around the contact area that encourage brittle fracture rather than plastic flow (Figs. 8 and 9). Fracture stresses and crack patterns (Fig. 9) can be evaluated by spherical indenters (26).

Vickers Hardness: The Vickers hardness indenter performs one of the most useful indentation measurements. The Vickers indenter is a small diamond pyramid with an angle of $136^{\circ}$ between faces (Table 3 and Fig. 5(a)). The indents are geometrically similar regardless of load, eliminating the variation of hardness with load except with very small loads $(20,27)$. The Vickers hardness $H_{V}$ is defined as the load divided by the surface area of the indent. Vickers hardness can be related to the yield strength of the material $\sigma_{y}$ by $H_{V}=2.78 \sigma_{y}$ for non-strain-hardening materials (20). The microhardness test is usually performed with the aid of a microscope, and only a very small specimen is required. 

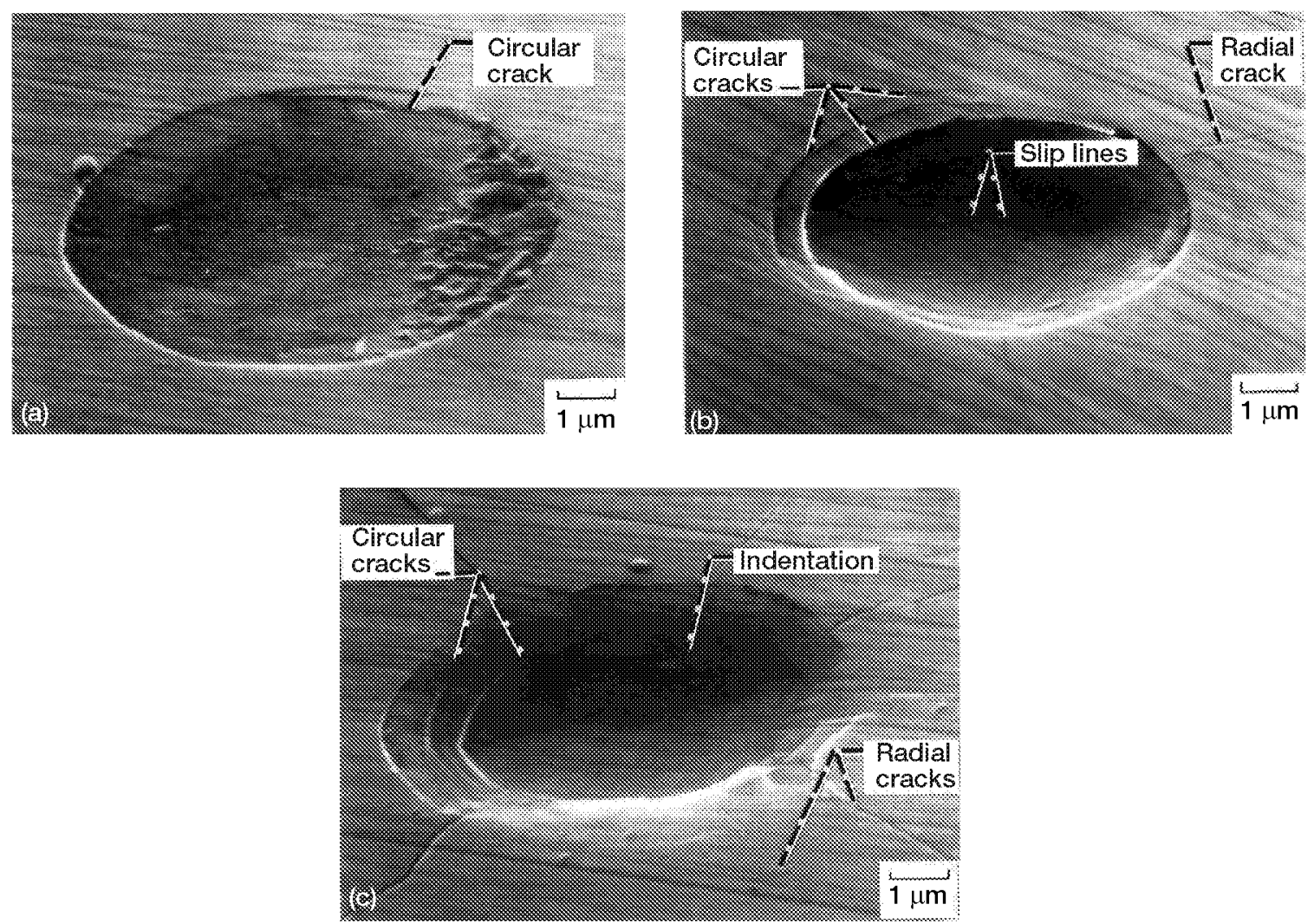

Figure 8.-Scanning electron micrographs of indentation and cracks on silicon carbide \{0001\} surface generated by hemispherical indenter. (a) Indenter radius, $0.1 \mathrm{~mm}$; load, $10 \mathrm{~N}$. (b) Indenter radius, $0.02 \mathrm{~mm}$; load, $5 \mathrm{~N}$. (c) Indenter radius, $0.008 \mathrm{~mm}$; load, $2 \mathrm{~N}$.

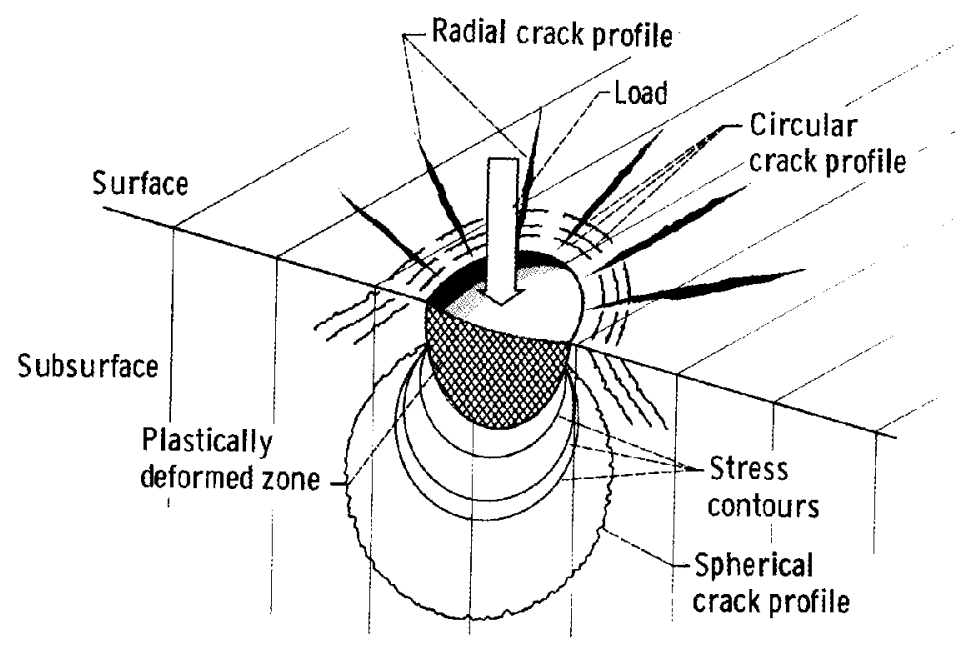

Figure 9.-Schematic of hemispherical crack formation under plastically deformed zone. 


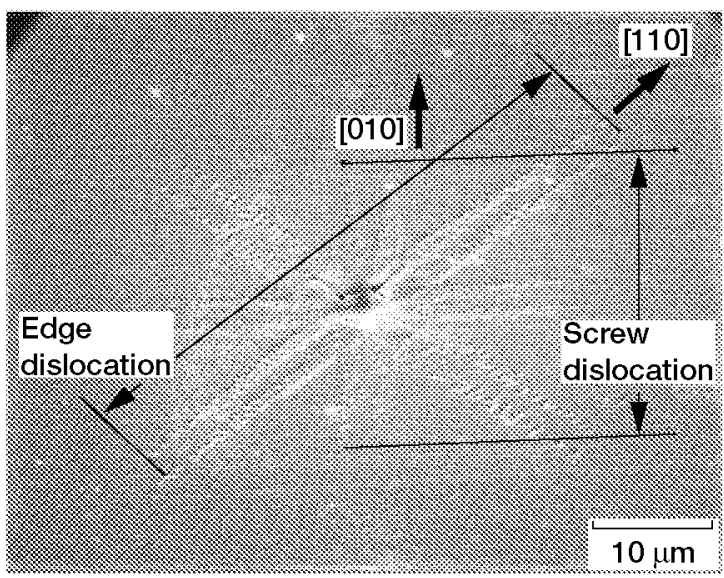

Figure 10.-Distribution of dislocation etch pits on MgO \{001\} surface around indentation made by Vickers diamond indenter at load of $0.1 \mathrm{~N}$.

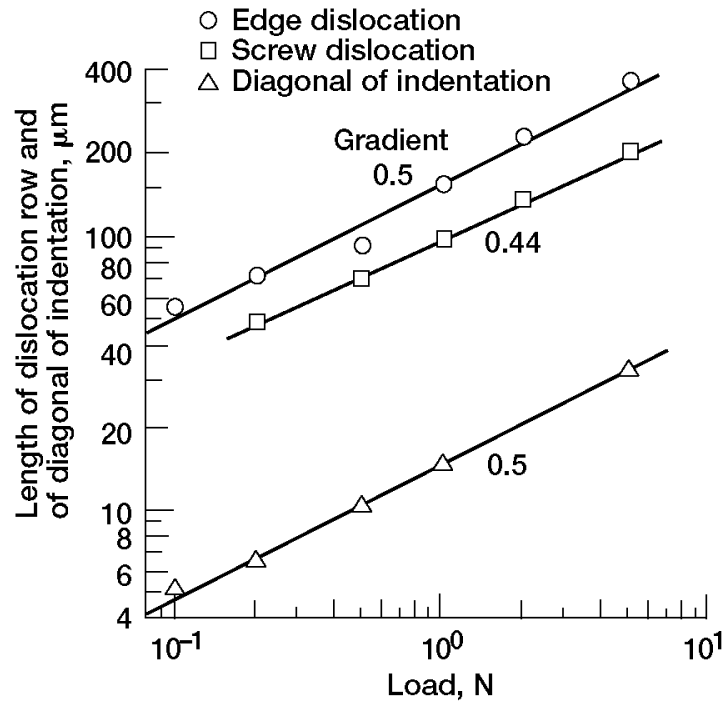

Figure $11 .-$ - Lengths of dislocation row and diagonal of indentation as function of load.

The indentation process imposes a considerable hydrostatic stress on the material, a great advantage when indenting brittle materials. The hydrostatic pressure suppresses fracture and makes an otherwise difficult measurement routine. In other types of mechanical tests, such as bend or tensile testing, careful machining is required so that surface defects do not create stress raisers and affect the test. The microhardness test also eliminates the difficulties associated with machine and fixture alignment.

Dislocation Phenomena: Figure 10 shows the distribution of dislocation etch pits on a well-defined, single-crystal magnesium oxide $(\mathrm{MgO})$ surface (28). The $\mathrm{MgO}$ bulk crystals were first cleaved along the $\{001\}$ surface in air and then subjected to hardness indentation in air at $298 \mathrm{~K}$, which introduced a certain amount of plastic deformation into the $\{001\}$ surface. Next, the $\mathrm{MgO}$ surfaces were chemically etched in a solution of five parts saturated ammonium chloride, one part sulfuric acid, and one part distilled water at room temperature. Then scanning electron micrographs were taken of the etched surfaces. The dislocation-etch-pit pattern on the indented surface (Fig. 10) contains screw dislocations in the [010] direction and edge dislocations in the [110] direction. The screw and edge dislocation arrays are 4.9 and 7.7 times wider, respectively, than the average length of the two diagonals of hardness indentation.

Figure 11 shows the length of the dislocation row and the length of the diagonal of indentation as functions of load on a log-log scale (28). As expected, the gradient of the diagonal length is approximately 0.5 because the Vickers hardness is independent of indentation load. Almost the same gradient is shown for the length of edge dislocations. However, the gradient for the screw dislocations is slightly smaller, possibly because cross slips occur easily at higher loads. The row of edge dislocations is always longer than that of screw dislocations for the hardness indentations.

Fracture Phenomena: Cracking and fractures around the indents can affect the accuracy of microhardness measurements. The energy absorbed by plastic deformation far exceeds that released by cracking for many materials (29). Although it can make accurate measurements difficult, indentation cracking can reveal important material parameters. Palmqvist was the first to realize that indentation cracking was related to the fracture toughness of the material (30).

Because cracks are formed around microhardness indents in several ways, different methods are needed to analyze them $(27,29-32)$. The cracks can be one of two basic types, median or lateral, as shown in Fig. 12 (27, 31, 32). Median cracks, which form on loading, are deep halfpenny-shaped cracks with the fracture plane normal to the surface. Lateral cracks, which form on unloading, are shallow cracks with a fracture plane approximately parallel to the surface. Lateral cracks are also known as Palmqvist cracks. Lawn and Marshall (33) reported a hardness of $10 \mathrm{GPa}$ and a fracture toughness of $0.6 \mathrm{MPa} \cdot \mathrm{m}^{1 / 2}$ 

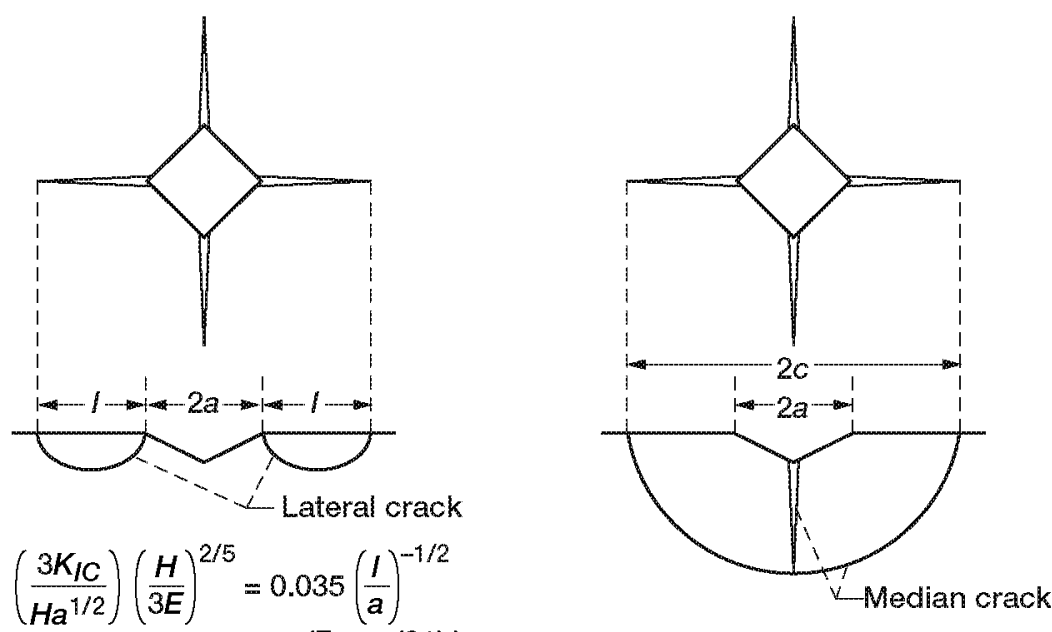

(From (31).)

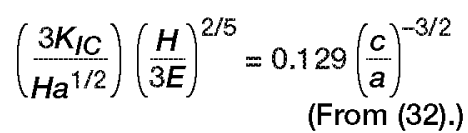

Figure 12.-Lateral and median crack geometry, where / is crack length, $a$ is indentation radius, $c$ is crack radius, $K_{I C}$ is fracture toughness, $E$ is Young's modulus, and $H$ is hardness.

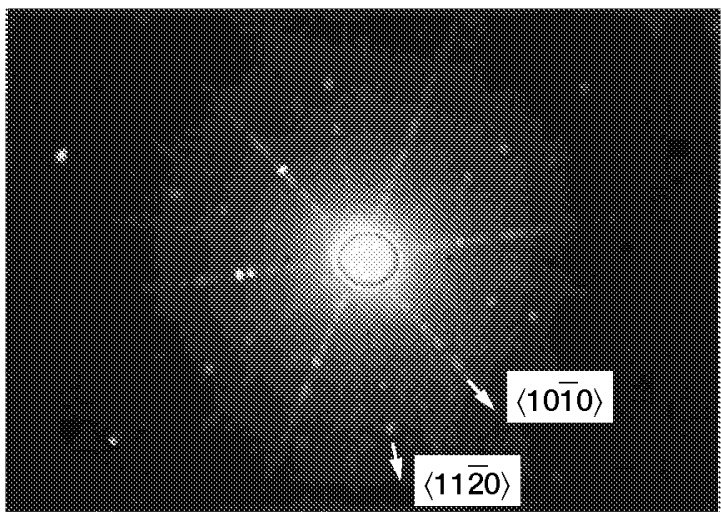

(a)

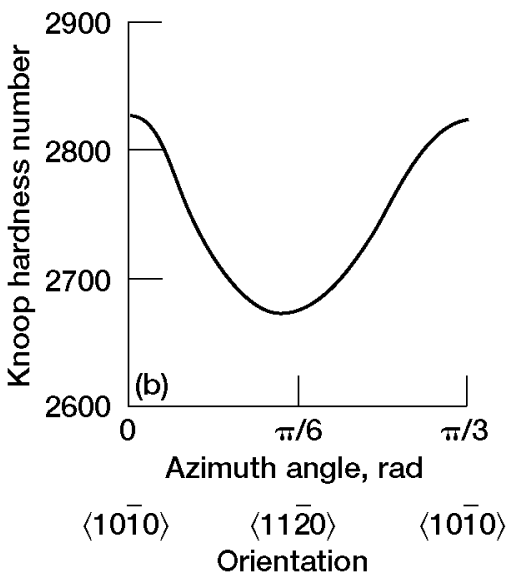

Orientation

Figure 13. - Indentation hardness anisotropy of SiC $\{001\}$ basal plane. Measuring load, $29 \mathbb{N}$. (a). Backreflection Laue photograph. (b) Knoop hardness as function of angle between long axis of Knoop indenter and $\langle 10 \overline{10}\rangle$ direction axis.

for silicon. Antis et al. (34) reported a hardness of $10.6 \mathrm{GPa}$ and a fracture toughness of $0.7 \mathrm{MPa} \cdot \mathrm{m}^{1 / 2}$ for silicon. All results were obtained at room temperature.

Crystallographic Orientation, Impurity, and Temperature Effects: As hardness is a conventionally used parameter for indicating the abrasion resistance of materials, it is useful and important to consider the effects of crystallographic orientation (hardness anisotropy), impurities, and temperature $(25,35-41$ ). Figure 13, for example, shows a backreflection Laue photograph and the results of Knoop hardness experiments made on the $\{0001\}$ plane of single-crystal silicon carbide $(\mathrm{SiC})$. The specimen is within $\pm 2^{\circ}$ of the low index $\{0001\}$ plane. The Knoop hardness is presented as a function of the orientation of the long axis of the Knoop diamond indenter with respect to the $\langle 10 \overline{1} 0\rangle$ direction at $10^{\circ}$ intervals. The hardness decreases smoothly to a minimum of approximately 2670 near $30^{\circ}$ from the $\langle 10 \overline{1} 0\rangle$ direction. Figure 13 indicates that the $\langle 10 \overline{1} 0\rangle$ direction is the maximum hardness direction and that the maximum hardness is approximately 2830 . The hardness results are consistent with those of other workers $(35,36)$. 


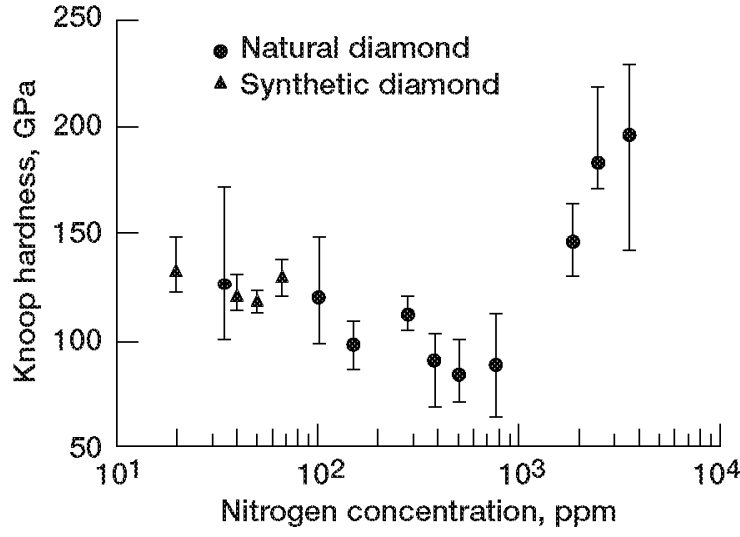

Figure 14.-Knoop harness as function of nitrogen concentration for synthetic and natural diamond. From Y Ma and GF Tellier, Rockwell Aerospace, Rocketdyne, Canoga Park, CA, 1994.

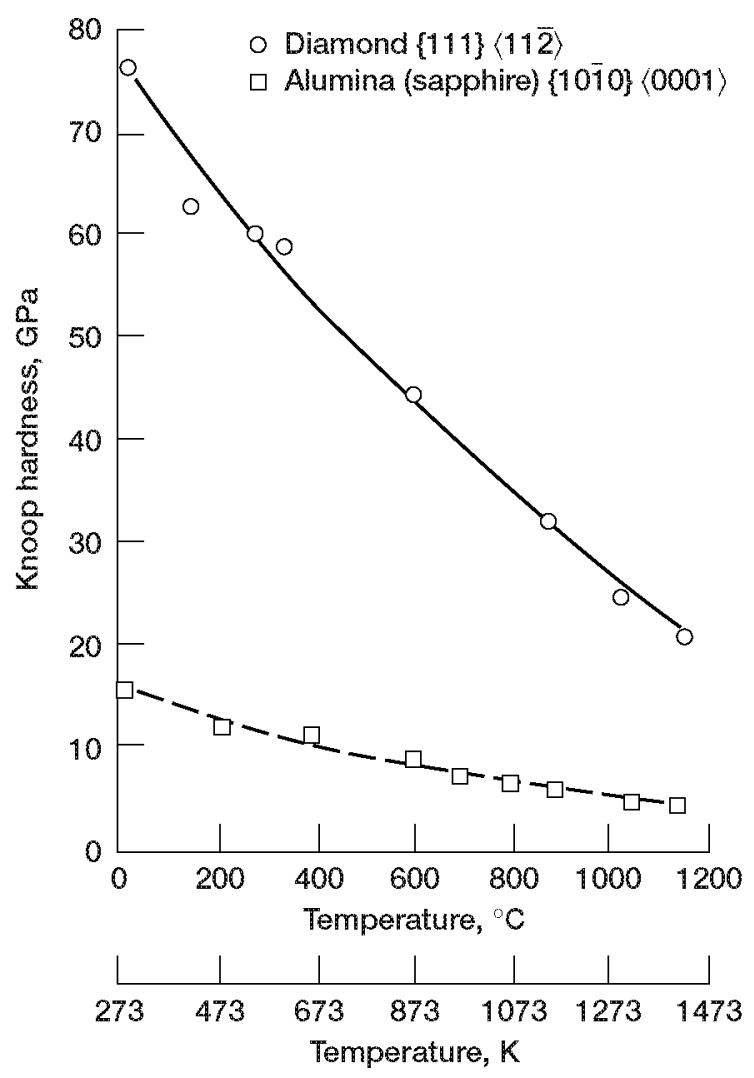

Figure $15 .-$ Knoop harness as function of temperature for diamond $\{111\}$ and sapphire (alumina) $\{1010\}$ surface. From (25).
Impurities considerably alter the indentation hardness, as shown in Fig. 14 (based on personal communication with Y Ma and GF Tellier, Rockwell Aerospace, Rocketdyne, Canoga Park, CA, 1994). Natural diamond invariably contains more nitrogen impurities and thus has greater ranges of hardness.

The hardness measured for diamond and sapphire is dependent on temperature, as shown in Fig. 15 (25). Hardness decreased with increasing temperature, and there was a corresponding increase in plastic deformation.

Hertzian Fracture by Spherical Indenter: Materials with high hardness are usually brittle. Knowledge of the fracture stresses and crack patterns produced in brittle materials, such as ceramics, is important in understanding materials behavior and tribological phenomena, such as wear, erosion, and abrasion. Such knowledge can be gained from crack patterns produced in brittle materials by spherical indenters under normal loading or sliding $(20,37$, 39). An indenter loaded onto the surface of a specimen generally produces a local damage zone (Hertzian cone crack or ring crack) comparable to the dimensions of the contact area (Fig. 16). These results have interest for two reasons. First, it is important to know a material's fracture behavior, particularly the crack patterns and fracture stresses under conditions arising in its tribological and mechanical applications. Second, indentation techniques are the main method for measuring the strength of materials.

Figure 17 shows well-developed ring cracks produced on silicon $\{100\}$, sapphire $\{0001\}$, and natural diamond $\{111\}$ during experiments in which the specimens were indented with $200-\mu \mathrm{m}$ - or $500-\mu \mathrm{m}$ radius spherical diamond indenters (39). Cleavage cracks emerging on the crystalline surface form inherent patterns on the surface. Cleavage occurred mostly along the $\{111\}$ planes in both silicon and diamond and along the $\{10 \overline{1} 2\}$ planes in sapphire. In silicon the ring cracks (following the $\{111\}$ cleavage planes) on the $\{100\}$ plane formed a square pattern in the $\langle 110\rangle$ direction. In sapphire the ring cracks (following the $\{10 \overline{1} 2\}$ cleavage planes) on the $\{0001\}$ plane formed a triangular or hexagonal pattern. In diamond the ring cracks (following the $\{111\}$ cleavage planes) on the $\{111\}$ plane formed a triangular or hexagonal pattern in the $\langle 110\rangle$ direction. 


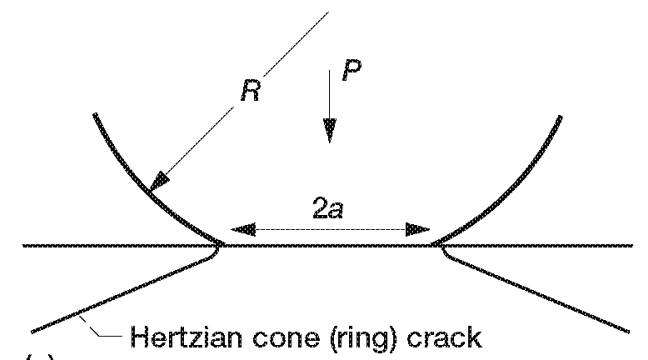

(a)

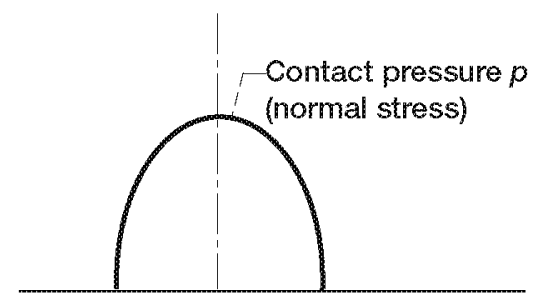

(c)

Figure 16.-Geometry of Hertzian cone (ring) crack and stress distributions formed by sphere loaded normally onto plane surface of brittle material.

(a) Hertzian ring crack. (b) Contact area. (c) Distribution of normal stress.

(d) Distribution of tensile stress.
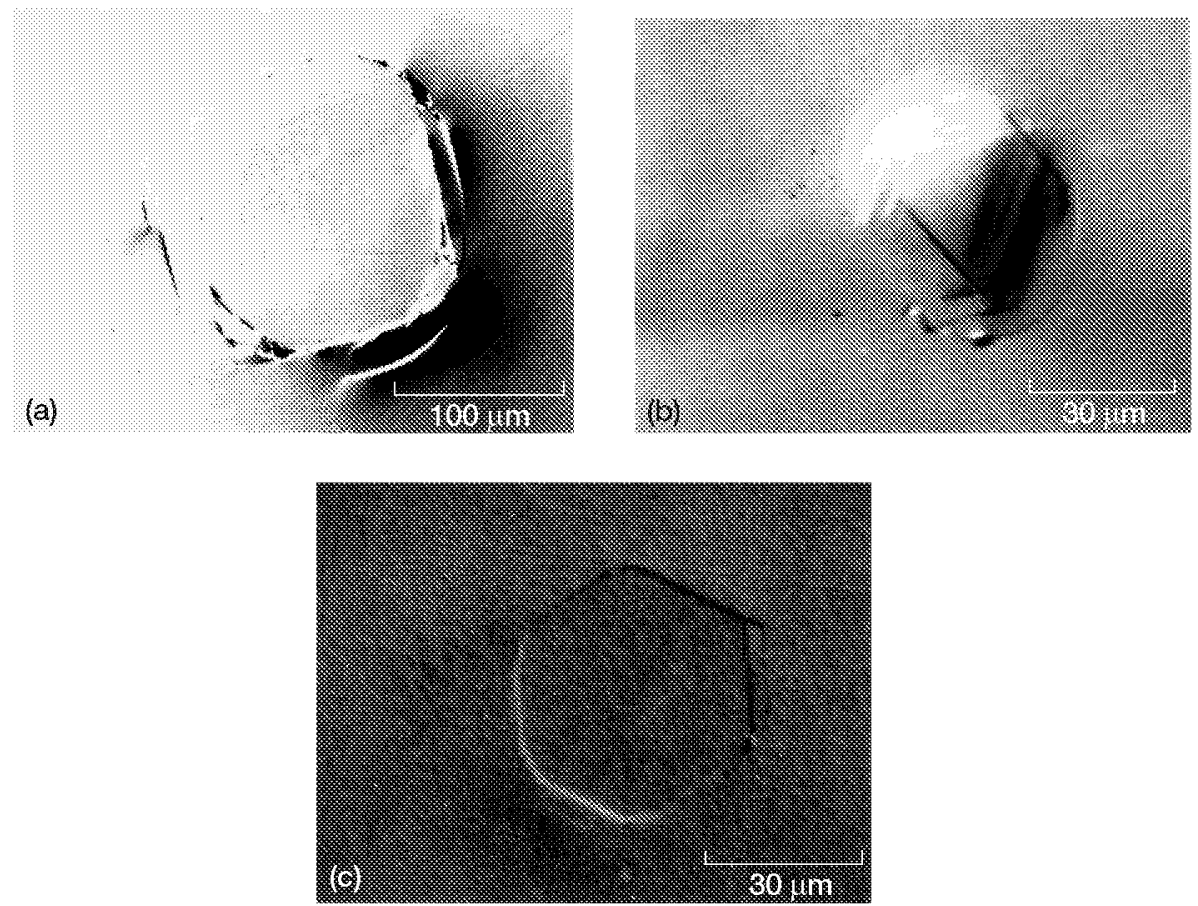

Figure 17.-Typical ring cracks. (a) Silicon $\{100\}$. Indenter radius, $500 \mu \mathrm{m}$. (b) Sapphire $\{0001\}$. Indenter radius, $200 \mu \mathrm{m}$. (c) Natural diamond $\{111\}$. Indenter radius, $200 \mu \mathrm{m}$. From (39). 


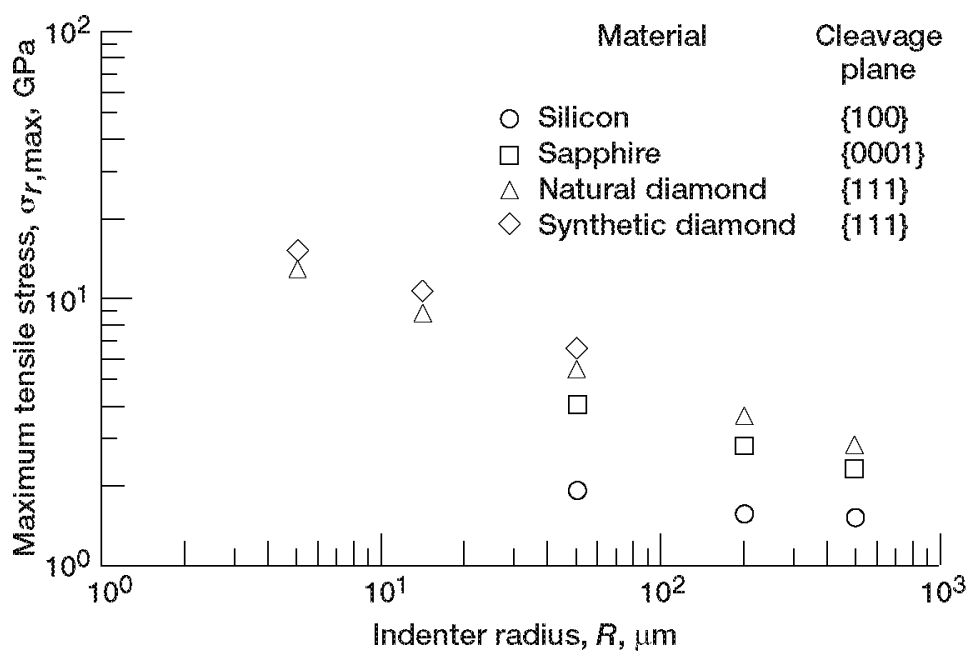

Figure 18.-Maximum tensile stress normal to cleavage plane at edge of contact circle as function of indenter radius for silicon, sapphire, and natural and synthetic diamond. From $(38,39)$.

Figure 18 presents the maximum tensile stress or the tensile strength, which acts normal to cleavage planes at the edge of the Hertzian contact circle and gives rise to fracture on silicon $\{100\}$, sapphire $\{0001\}$, natural diamond $\{111\}$, and synthetic diamond $\{111\}$ as a function of indenter radius $(38,39)$. Indenter radius had a marked effect on Hertzian fracture and on the maximum tensile stress to fracture. The greater the indenter radius, the lower the maximum tensile stress to fracture. Further, the maximum tensile stresses (tensile strengths) shown in Fig. 18 are of the same order as the theoretical strengths for the corresponding materials. The theoretical strengths are approximately 1/10th of the Young's moduli, which are $167 \mathrm{GPa}$ for silicon, $380 \mathrm{GPa}$ for sapphire, and $1020 \mathrm{GPa}$ for natural and synthetic diamond. The tensile strengths of the materials investigated herein were, in ascending order, silicon, sapphire, natural diamond, and synthetic diamond.

Impurities considerably alter the tensile strength. For example, when the $\{100\}$ surfaces of single-

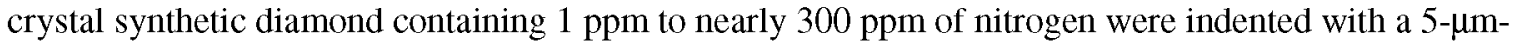
radius spherical diamond indenter, nitrogen impurities altered the maximum tensile stress to fracture (tensile strength). The tensile strength decreased as nitrogen concentration increased (39).

4.2.3 Scratch Microhardness Measurement.-Any scratch hardness test is a form of controlled abrasive wear, and thus it seems reasonable to use the test as a means of ranking materials for their likely resistance to abrasion in service (42). Scratch microhardness measurements and surface morphological studies are widely accepted by industry and researchers for the quantitative measurement of the adhesion of thin films and the ductility and abrasion resistance (plasticity) of materials. They are rapid and simple and allow direct comparison between similar coatings, surface-modified materials, or engineering materials. Scratch testing is most often used as a quality control technique enabling the performance of one surface to be qualitatively and, to some extent, quantitatively compared with another that is known to be satisfactory in use. The scratch test is especially popular when dealing with surfaces that have been engineered by thermal, chemical, or coating treatments to enhance hardness or wear resistance.

A diamond stylus or related hard stylus is used to make a scratch measurement on a specimen. Controlled stress and strain are induced on the surface. The mechanical response can be measured by the surface morphological changes and the friction monitored during scratching. Subsequent Raman mapping (Table 1(c)) of the scratched region can provide information on the local stress and strain distribution, together with the molecular composition of any chemical species and its changes induced by scratching action. 

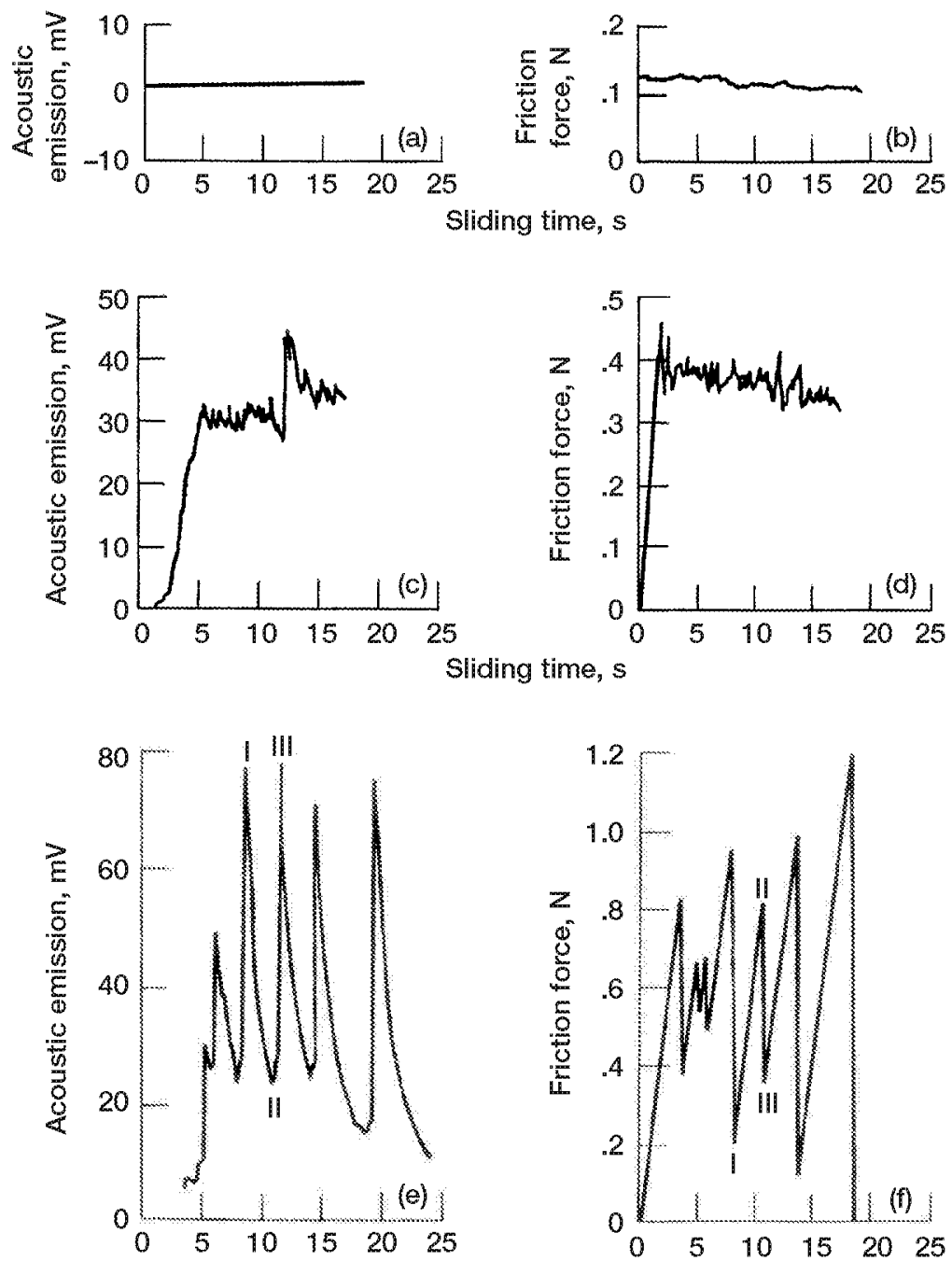

Sliding time, $s$

Figure 19.-Typical acoustic emission traces and friction force traces for ion-beam-deposited BN film in contact with hemispherical diamond film in laboratory air.

Investigators have detected released acoustic emissions when the intrinsic cohesive bonds in the coating film or the adhesive bonds between the film and the substrate, or both, are broken and a new surface is created. The pattern and intensity of the acoustic emissions depend on the nature of the disturbance (i.e., plastic flow, cracking, or flaking of fragments $(43,44)$ ). For example, Fig. 19 presents typical acoustic emission traces and friction force traces for a boron nitride $(\mathrm{BN})$ film deposited onto a silicon substrate (45). When the BN film surface is brought into contact with a diamond pin (tip radius of $0.2 \mathrm{~mm}$ ) under a small load (lower than the critical loads needed to fracture intrinsic cohesive bonds in the BN film and adhesive bonds between the film and the substrate), no acoustic emission is detected (Fig. 19(a)). The friction force trace at the same load $(3 \mathrm{~N})$ fluctuates slightly with no trace of stick-slip behavior (Fig. 19(b)). After the diamond has passed over the surface once, scanning electron microscopic examination of the wear track indicates that a permanent groove is formed in the BN film, much as occurs in metallic films under similar conditions. However, no cracking of the BN film is observed with sliding. 

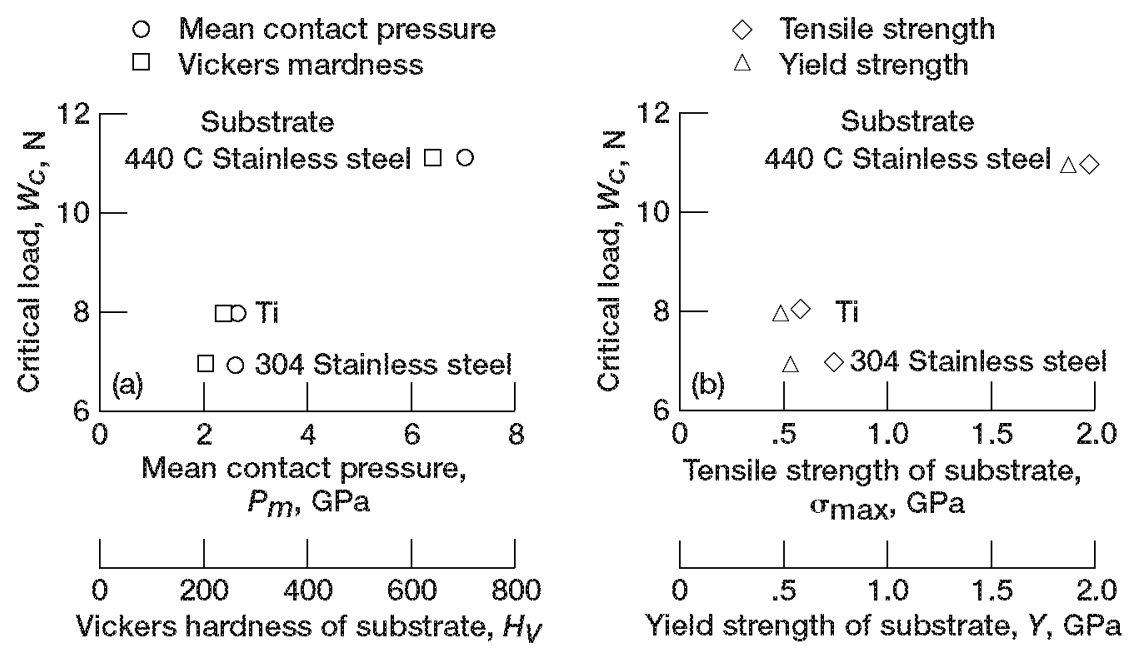

Figure 20.-Critical load needed to fracture BN film and interfacial adhesive bonds between film and substrate in sliding contact with hemispherical diamond film in laboratory air (mean contact pressure is scratch hardness of substrate).

Increasing the load to or above the critical loads required to fracture the BN film and the interfacial adhesive bonds between the film and the substrate results in a small amount of cracking in and near the plastically deformed groove (45). The acoustic emission trace detected at a load of $9 \mathrm{~N}$ indicates evidence of a fluctuating acoustic emission signal output (Fig. 19(c)). Acoustic emission is observed when the sliding appears to involve small amounts of cracking in addition to plastic flow. Such acoustic emission is caused by the release of elastic energy when cracks propagate in the $\mathrm{BN}$ film. The friction force trace measured at the $9-\mathrm{N}$ load is characterized by randomly fluctuating behavior, but only occasional evidence of stick-slip behavior is observed (Fig. 19(d)).

When a much higher load (e.g., $12 \mathrm{~N}$ ) is applied to the BN film, the sliding action produces, in addition to plastic flow, locally gross surface and subsurface fracturing in the film and at the interface between the BN film and the substrate (45). In such cases the acoustic emission traces are primarily characterized by chevron-shaped peaks (Fig. 19(e)), whereas the friction force traces are primarily characterized by continuous, marked stick-slip behavior (Fig. 19(f)).

The behavior of acoustic emission is related to that of friction force. For example, at point I in Figs. 19(e) and (f) the diamond pin comes to rest until point II is reached. At point II the pin is set into motion, slips, and continues to move until point III is reached. Acoustic emission is released at point II because the slip action produces fracturing at the interface between the BN film and the substrate. At point III the pin comes to rest again. Thus, fracture in the film and at the interface between the BN film and the substrate is responsible for the observed acoustic emission signal output and friction behavior. Acoustic and friction measurements of the critical load required to fracture a ceramic film on a substrate agree well with the critical loads detected by optical and scanning electron microscopy of the scratches.

In another example, Fig. 20 presents the critical loads required to fracture a BN film and the adhesive bonds between the film and various metallic substrates as determined by acoustic emission and friction force measurements (45). The critical load to fracture is related to the hardness and strength of the metallic substrate. The harder the substrate or the greater its strength, the higher the critical load. Benjamin and Weaver (46) derived the following expressions for scratch adhesion in terms of shear stress $S$ produced at the coating/substrate interface by the plastic deformation, the hardness of the substrate (mean contact pressure at fully plastic state $P_{m}$ ), the critical load applied on the pin $W_{c}$, the tip radius of the pin $R$, and the width of the resulting scratch $D$ : 
TABLE 5.CRITICAL NORMAL LOAD TO FRACTURE BN FILM IN SLIDING CONTACT AND SHEAR STRENGTH OF INTERFACIAI ADHESIVE BONDS

\begin{tabular}{|c|c|c|c|c|c|c|}
\hline \multirow[t]{2}{*}{ Substrate } & \multicolumn{2}{|c|}{$\begin{array}{l}\text { Vickers hardness, } \\
\qquad \mathrm{H}_{V} \\
\mathrm{GPa}\end{array}$} & \multirow{2}{*}{$\begin{array}{c}\text { Mean contact } \\
\text { (yield) pressure at } \\
\text { fully plastic state, } \\
P_{m} \\
\text { GPa }\end{array}$} & \multirow{2}{*}{$\begin{array}{c}\text { Critical } \\
\text { nomal } \\
\text { load, } \\
W_{c} \\
N\end{array}$} & \multicolumn{2}{|c|}{$\begin{array}{c}\text { Interfacial shear strength, } \\
\qquad, \\
\text { GPa }\end{array}$} \\
\hline & Substrate & $\begin{array}{l}\text { BN } \\
\text { film }\end{array}$ & & & $S=K\left(\frac{W_{c} P_{m}}{\pi R^{2}}\right)^{1 / 2}$ & $S \equiv \frac{2 W_{c}}{\pi D R}$ \\
\hline Silicon & ${ }^{6} 8.89$ & ${ }^{b} 18.8$ & 24.0 & 11 & 1.1 & 1.0 \\
\hline $\begin{array}{l}440 C \text { stainless } \\
\text { stee } 1\end{array}$ & $a 7.1$ & ${ }^{\mathrm{b}} 10.2$ & 12.0 & 11 & .77 & .68 \\
\hline $\begin{array}{l}304 \text { stainless } \\
\text { steel }\end{array}$ & $a_{2.5}$ & $b_{4.3}$ & 4.1 & 7 & .37 & .29 \\
\hline Titanium & $\mathrm{a}_{2} .6$ & $b_{3.4}$ & 3.3 & 8 & .40 & .30 \\
\hline
\end{tabular}

Hardness measuring load, $2 \mathrm{~N}$.

${ }^{b}$ Hardness measuring load, $0.05 \mathrm{~N}$.

$$
\begin{gathered}
S=K\left(\frac{W_{c} P_{m}}{\pi R^{2}}\right)^{1 / 2} \\
S \cong \frac{2 W_{c}}{\pi D R}
\end{gathered}
$$

These relationships allow for the calculation of the shear strength (i.e., the adhesion strength of the interfacial bonds) $(46,47)$. The results are presented in Table 5 . The values of the critical loads were obtained and confirmed not only by optical and scanning electron microscopic examination of the scratches but also by the acoustic emission technique. Table 5 reveals the strong correlation between the shear strength (i.e., the adhesion strength) and the hardness of the substrate. The harder the metallic substrate, the greater the shear strength.

If a hard material is brought into contact with a softer single crystal, the plastic deformation and accordingly the coefficient of friction of the single-crystal material are anisotropic and relate to the crystal structure. In crystals of comparable purity and crystallographic perfection, slip always begins when the shear stress across the slip planes reaches a certain definite value known as the critical resolved shear stress. The actual stress required to start deformation depends on the orientation of the slip planes relative to the applied stress. The details on the anisotropic plastic deformation and hardness of single-crystal ceramics as they relate to friction can be found in the references $(48,49,50)$.

\section{Elemental Composition and Chemical State of Solid Surfaces}

Elemental composition is perhaps the most basic information about materials, followed by chemical state information, phase identification, the determination of structure (bond structure, atomic sites, bond lengths, and angles), and defects (Tables 1(c) and (d)). The elemental and chemical state, phase, microstructure, and defects of a solid often vary as a function of depth into the material or spatially across the material. Many techniques specialize in addressing these variations down to extremely fine dimensions, as small as on the order of angstroms in some cases (refer to Fig. 21). Requests are made for physical and chemical information as a function of depth, to depths of $1 \mathrm{~mm}$ or so (materials have about 3 million atomic layers per millimeter of depth). This upper region at the material surface affects a broad spectrum of properties: elemental composition, contamination, adhesion, bonding, corrosion, surface strength and toughness, hardness, chemical activity, friction, wear, and lubrication. Knowing these variations is of great importance when selecting and using coatings, modified surfaces, and materials. 


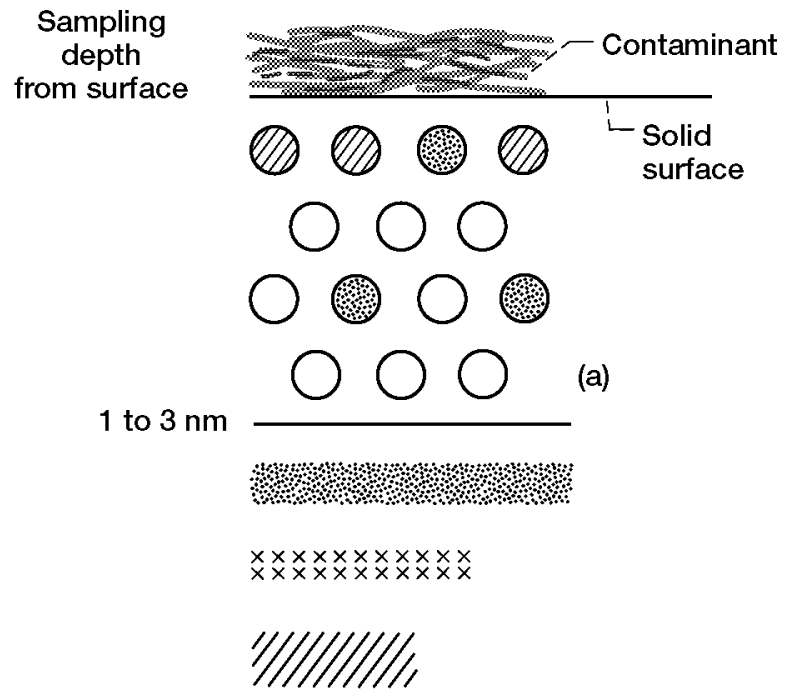

(b)

10 to $30 \mathrm{~nm}$
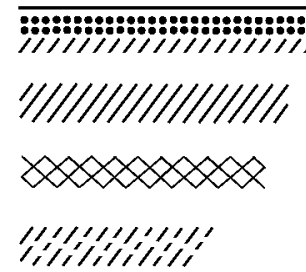

$100 \mathrm{~nm}$ to $1 \mu \mathrm{m}$

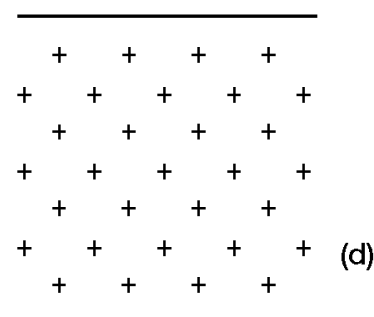

$1 \mathrm{~mm}$

Figure 21. - Schematic diagram showing regimes of (a) surface analysis, (b) thin film analysis, (c) interface analysis, and (d) bulk substrate analysis.
For surfaces, interfaces, and thin films there is often little material to analyze-hence, the need for many microanalytical methods such as those listed in Table 1. Within microanalysis it is often necessary to identify trace components down to extremely low concentrations (parts per trillion in some cases), and a number of techniques specialize in this aspect. In other cases a high degree of accuracy in measuring the presence of major components may be of interest. Usually, the techniques that are good for trace identification do not accurately quantify major components. Most complete analyses require the use of multiple techniques, the selection of which depends on the nature of the specimen and the desired information.

It is important to know the sampling (information) depth of the analytical tool to be used. For example, let us compare the analysis of two different specimens. The first has an atomic layer of one or more impurities on the surface; the other has these atoms distributed homogeneously within the specimen. When a conventional $x$-ray microprobe with a sampling depth of about $1000 \mathrm{~nm}$ is used, the specimens produce signals of equal intensity and it is not possible to differentiate between them. One can sometimes get an indication of whether there is a bulk impurity or a surface segregation (or a thin film) by lowering the electron beam voltage or by measuring at grazing incidence. However, this is not possible with only a few atomic layers. On the other hand, because of their insufficient detection limits Auger electron spectroscopy (AES) or $\mathrm{x}$-ray photoelectron spectroscopy (XPS) will produce two quite different spectra: in the first

specimen, a strong signal from the impurity layer caused by the low sampling depth; in the second specimen, only the spectrum of the pure bulk material with no indication of the impurities.

Ion scattering spectroscopy (ISS) and secondary ion mass spectrometry (SIMS) are extremely surface-sensitive characterization tools, followed by AES and XPS (or electron spectroscopy for chemical analysis (ESCA)). Electron probe microanalysis (EPMA) and energy-dispersive $\mathrm{x}$-ray spectroscopy analysis (EDS) are bulk characterization tools. Thus, knowing both the structure of real surfaces and the capabilities of the various characterization techniques is of great importance.

It was during the 1960's that the amazing growth and diversification of surface analytical techniques began and evolved with the development of two types of ultra-high-vacuum electron spectroscopy-AES closely followed by XPS. The combination of the all-encompassing definition of surface engineering and tribology with these surface analytical techniques, including a variety of electronic, photonic, and ionic spectroscopies and microscopies, reflects the trend of surface engineering and tribology today. 
In the following subsections characterization equipment commonly used in the fields of surface modifications, thin films and coatings, and tribology and examples of their use are described. Also, it is shown where surface science can play a role in advancing our knowledge of adhesion, friction, lubrication, and wear as well as the basics of surface phenomena.

A number of techniques are now available for measuring the composition of any solid surface. The most widely used techniques for surface analysis are AES, XPS (or ESCA), and SIMS (Table 1(c)). These techniques are well suited for examining extremely thin layers, including the contaminant layers and the oxide layers.

\subsection{SEM and EDS}

The single most useful tool available today to surface engineers and scientists, tribologists, and lubrication engineers interested in studying the morphology, defects, and wear behavior of material surfaces is undoubtedly the scanning electron microscope (SEM). Especially, the combination of the SEM and $\mathrm{x}$-ray analysis using either energy-dispersive $\mathrm{x}$-ray spectroscopy (EDS) or wavelength-dispersive $\mathrm{x}$-ray spectroscopy (WDS) provides a powerful tool for local microchemical analysis $(7,51)$. The use of electron microprobe techniques in the SEM is now a well-established procedure. The two techniques, EDS and WDS, differ only in the use of an energy-dispersive, solid-state detector versus a wavelength-dispersive, crystal spectrometer. Successful studies have been carried out to characterize surface-modified materials, thin films and coatings, and the surfaces of bulk materials. The SEM is often the first analytical instrument used when a quick look at a material is required and the light microscope no longer provides adequate spatial resolution or depth of focus. The SEM provides the investigator with a highly magnified image of a material surface. Its resolution can approach a few nanometers, and it can be operated at magnifications from about $10 \mathrm{X}$ to $300000 \mathrm{X}$. The SEM produces not only morphological and topographical information but information concerning the elemental composition in near-surface regions.

In the SEM an electron beam is focused into a fine probe and subsequently raster scanned over a small rectangular area. As the electron beam interacts with the specimen, it creates various signals, such as secondary electrons, internal currents, and photon emissions, all of which can be collected by appropriate detectors. The SEM-EDS produces three principal images: secondary electron images, backscattered electron images, and elemental $\mathrm{x}$-ray maps. Secondary and backscattered electrons are conventionally separated according to their energies. They are produced by different mechanisms. When a high-energy primary electron interacts with an atom, it undergoes either inelastic scattering with the atomic electrons or elastic scattering with the atomic nucleus. In an inelastic collision with an electron some amount of energy is transferred to the other electron. If the energy transferred is extremely small, the emitted electron will probably not have enough energy to exit the surface. If the energy transferred exceeds the work function of the material, the emitted electron will exit the solid. When the energy of the emitted electron is less than about $50 \mathrm{eV}$, by convention it is referred to as a secondary electron (SE), or simply a secondary. Most of the emitted secondaries are produced within the first few nanometers of the surface. Secondaries produced much deeper in the material suffer additional inelastic collisions, which lower their energy and trap them in the interior of the solid.

Higher energy electrons are primary electrons that have been scattered without loss of kinetic energy (i.e., elastically) by the nucleus of an atom, although these collisions may occur after the primary electron has already lost some energy to inelastic scattering. Backscattered electrons (BSE's) are, by definition, electrons that leave the specimen with only a small loss of energy relative to the primary electron beam energy, but BSE's are generally considered to be the electrons that exit the specimen with an energy greater than $50 \mathrm{eV}$, including Auger electrons. The BSE imaging mode can be extremely useful for tribological applications, since the energy, spatial distribution, and number of BSE's depend on the effective atomic number of the specimen, its orientation with respect to the primary beam, and the surface condition. The backscatter coefficient, or relative number of electrons leaving the specimen, increases with 
increasing atomic number (51). The higher the atomic number $Z$ of a material, the more likely it is that backscattering will occur. Thus, as a beam passes from a low $Z$ to a high- $Z$ area, the signal due to backscattering and consequently the image brightness will increase. There is a built-in contrast caused by elemental differences. BSE images can therefore be used to distinguish different phases, modified surfaces, thin films and coatings, and foreign species of the specimen having different mean atomic numbers (atomic number contrast). For most specimens examined in SEM, except for those that are flat or polished, the specimen both varies in chemistry from area to area and exhibits a varying rough surface. As a result, both atomic number and topographic contrast are present in the BSE signal (as well as in the SE signal). In general, if the high-energy BSE's are collected from the specimen at a relatively high takeoff angle, atomic number information is emphasized. Conversely, if the high-energy electrons leaving the specimen are collected at a relatively low takeoff angle, topographic information is emphasized. For nearly all BSE applications the investigator is interested in the atomic number contrast and not in the topographic contrast. Note that the backscatter coefficient is defined as the number of BSE's emitted by the specimen for each electron incident on the specimen. Because of the relatively deep penetration of the incident electron beam combined with the extensive range of the BSE's produced, spatial resolution in the BSE mode is generally limited to about $100 \mathrm{~nm}$ in bulk specimens under the usual specimen/ detector configurations.

Both energy-dispersive and wavelength-dispersive $\mathrm{x}$-ray detectors can be used for element detection in the SEM. When the atoms in a material are ionized by high-energy radiation, usually electrons, they emit characteristic $x$-rays. The detectors produce an output signal that is proportional to the number of $\mathrm{x}$-ray photons in the area under electron bombardment. EDS is a technique of $\mathrm{x}$-ray spectroscopy that is based on the collection and energy dispersion of characteristic x-rays. Most EDS applications are in electron column instruments like the SEM, the electron probe microanalyzer (EPMA), and the transmission electron microscope (TEM). X-rays entering a solid-state detector, usually made from lithium-drifted silicon, in an EDS spectrometer are converted into signals that can be processed by the electronics into an $\mathrm{x}$-ray energy map or an $\mathrm{x}$-ray energy histogram. A common application of the $\mathrm{x}$-ray systems, such as EDS and WDS, involves $x$-ray mapping, in which the concentration distribution of an element of interest is displayed on a micrograph. The detectors can be adjusted to pass only the pulse range corresponding to a particular element. This output can then be used to produce an x-ray map or an elemental image. Higher concentrations of a particular element yield higher $x$-ray photon pulse rates, and the agglomeration of these pulses, which appear as dots in the image, generate light and dark areas relating to the element's concentration distribution. In $\mathrm{x}$-ray spectroscopy the $\mathrm{x}$-ray spectrum consists of a series of peaks that represent the type and relative amount of each element in the specimen. The number of counts in each peak can be further converted into elemental weight concentration either by comparison with standards or by standardless calculations. Three modes of analysis are commonly used: spectrum acquisition, spatial distribution or dot mapping of the elements, and element line scans.

\subsection{AES and XPS}

The surface analytical techniques most commonly used in surface engineering and tribology are AES and XPS (or ESCA). Each can determine the composition of the outermost atomic layers of a clean surface or of surfaces covered with adsorbed gas films, oxide films, thin films and coatings, reaction film products, surface-modified materials, and frictionally transferred films $(1,4-8,52,53)$.

AES and XPS are generally called "surface analysis" techniques, but this term can be misleading (8, 9). Although these techniques derive their usefulness from their intrinsic surface sensitivity, they can also be used to determine the composition of deeper layers. Such a determination is normally achieved through controlled surface erosion by ion bombardment. AES or XPS analyzes the residual surface left after a certain sputtering time with rare gas ions. In this way composition depth profiles can be obtained that provide a powerful means for analyzing thin films, surface coatings, reaction film products, transferred films, 
and their interfaces. Clearly, this capability also makes AES and XPS ideal for studying surface-modified materials and wear-resistant coatings. There are, however, a number of practical differences between the two techniques (e.g., detection speed, background, and spatial resolution) that are generally more advantageous in AES profiling. AES uses a focused electron beam to create secondary electrons near a solid surface. Some of these electrons (the Auger electrons) have energies characteristic of the elements.

As stated above, AES can characterize the specimen in depth and provide elemental depth profiles when used in combination with sputtering (e.g., argon-ion sputter etching) to gradually remove the surface. In addition to energies characteristic of the elements, some of the Auger electrons detected have energies characteristic, in many cases, of the chemical bonding of the atoms from which they are released. Because of their characteristic energies and the shallow depths from which they escape without energy loss, Auger electrons can characterize the elemental composition and, at times, the chemistry of surfaces. The Auger peaks of many elements show significant changes in position or shape in different chemical conditions and environments.

Thus, AES has the attributes of high lateral resolution, relatively high sensitivity, and standardless semi-quantitative elemental analysis. It also provides chemical bonding information in some cases. Further, the high spatial resolution of the electron beam and the sputter etching process allow microanalysis of three-dimensional regions of solid specimens.

In XPS, mono-energetic soft $\mathrm{x}$-rays bombard a specimen material, causing electrons to be ejected. The elements present in the specimen can be identified directly from the kinetic energies of these ejected photoelectrons. Electron binding energies are sensitive to the chemical state of the atom. Although XPS is designed to deal with solids, specimens can be gaseous, liquid, or solid. XPS is applicable to metals, ceramics, semiconductors, and organic, biological, and polymeric materials. Although x-ray beam damage can sometimes be significant, especially in organic materials, XPS is the least destructive of all the electron or ion spectroscopy techniques. The depth of solid material sampled varies from the top 2 atomic layers to 15 to 20 layers. This surface sensitivity, combined with quantitative and chemical analysis capabilities, has made XPS the most broadly applicable general surface analysis technique used today, especially in the field of tribology. Like AES, XPS can also characterize the specimen in depth and provide elemental depth profiles when used in combination with sputtering (e.g., argon-ion sputter etching) to gradually remove the surface.

In general, AES provides elemental information only. The AES peaks of many elements, however, show significant changes in position or shape in different chemical environments. On the other hand, the main advantage of XPS is its ability to provide chemical information from the shifts in binding energy. The particular strengths of XPS are quantitative elemental analysis of surfaces (without standards) and chemical state analysis. For a solid, AES and XPS probe 2 to 10 and 2 to 20 atomic layers deep, respectively, depending on the material, the energy of the photoelectron concerned, and the angle (with respect to the surface) of the measurement.

The thickness of the outer monatomic layers can be determined by studying the attenuation of photoelectrons originating in the bulk material caused by the layers and by studying the variation in intensity of photoelectrons emitted by the layers as a function of thickness.

\section{Case Study: Characterization of Diamond Films and Coatings}

\subsection{Introduction and Background}

During the last decade significant progress has been made in the development of advanced surface films and coatings for engineering and biomedical applications. Some of the most exciting recent developments are superhard coatings and films, such as chemical-vapor-deposited (CVD) diamond, diamondlike carbon (DLC), carbon nitride $\left(\mathrm{CN}_{\mathrm{x}}\right)$, and cubic boron nitride (c-BN) (54). 
The commercial potential of CVD diamond films has been established, and a number of applications have been identified through university, industry, and government research studies. CVD diamond is presently produced in the form of coatings or wafers. CVD diamond film technology offers a broader technological potential than do natural and high-pressure synthetic diamond because size, geometry, and cost will not be as limiting. Diamond coatings can improve many of the surface properties of engineering substrate materials, including erosion, corrosion, and wear resistance (54). Examples of actual and potential applications, such as microelectromechanical systems and environmentally durable barriers, of diamond coatings and related superhard coatings are described in a reference (55). For example, diamond coatings can be used as a chemical and mechanical barrier for space shuttle check valves, particularly guide pins and seat assemblies (56).

Achieving the quality and distinctive properties of diamond coatings and films requires optimizing deposition parameters through the study of the physical, chemical, and structural changes of coatings and films as a function of deposition parameters. These parameters must not only give the appropriate initial level of quality and properties but must also provide durable coatings and films.

For a material to be recognized as diamond it must have all of the following characteristics $(57,58)$ :

1. Crystalline diamond morphology and microstructure visible by optical or electron microscopy

2. Single-phase diamond crystalline structure detectable by $\mathrm{x}$-ray or electron diffraction

3. Clear, sharp diamond peak at $1332 \mathrm{~cm}^{-1}$ in a Raman spectrum

4. Carbon content (>95 at. \%)

5. Low coefficient of friction (0.01 to 0.05$)$ in air, but high coefficient of friction $(>0.4)$ in ultrahigh vacuum

This section deals with the application of measurement and characterization techniques required for the technological growth of advanced CVD diamond films and coatings. First, CVD diamond film deposition technology is briefly described and then the measurement and characterization techniques of greatest interest are reviewed.

Each measurement and characterization technique provides unique information. A combination of techniques can provide the technical information required to understand the quality and properties of CVD diamond films, which are important to their application in specific component systems and environments. In this study the combination of measurement and characterization techniques was successfully applied to correlate deposition parameters and resultant diamond film composition, crystallinity, grain size, surface roughness, and coefficient of friction. An important case study of microwave-plasmaassisted CVD diamond films will be highlighted. Some earlier data and experimental details on this research are given in the references $(59,60,61)$.

\subsection{CVD Diamond Film Deposition Technology}

The basic reaction in the chemical vapor deposition of diamond is simple (57). It involves the decomposition of a hydrocarbon, such as methane, as follows:

$$
\mathrm{CH}_{4} \rightarrow(\text { Activation }) \rightarrow \mathrm{C}(\text { diamond }) \rightarrow 2 \mathrm{H}_{2}
$$

The carbon species must be activated, since graphite at low pressure is thermodynamically stable and, without activation, only graphite would be formed. Activation is obtained by using either high temperature or plasma, each of which requires a great deal of energy. The four most often used activation methods at this time are 
1. High-frequency, plasma glow discharge using the microwave and radiofrequency processes

2. Plasma arcing using the direct-current arc and radiofrequency arc processes

3. Thermal chemical vapor deposition using the hot-filament process

4. Combustion synthesis using an oxyacetylene torch

Plasma arcing and combustion synthesis have high deposition rates $(57,62$, and personal communication with $\mathrm{H}$ Windischmann). For example, the mass deposition rate using a direct-current arc jet reached 20 carats/hr in 1997 (62 and personal communication with $\mathrm{H}$ Windischmann). Progress in direct-current arc jet deposition has advanced to the point that the $\$ 5 / \mathrm{carat}\left(\$ 8 / \mathrm{cm}^{2}\right)$ barrier has been breached. In 1997 diamond made by direct-current arc jet was available at $\$ 8 / \mathrm{cm}^{2}\left(\$ 50 / \mathrm{in}^{2}\right)$.

Diamond has been deposited from a large variety of precursors, including methane, aliphatic and aromatic hydrocarbons, alcohols, ketones, and solid polymers such as polyethylene, polypropylene, and polystyrene (57). These substances generally decompose into two stable primary species, the methyl radicals $\left(\mathrm{CH}_{3}\right)$ and acetylene $\left(\mathrm{C}_{2} \mathrm{H}_{4}\right)$. The radical is the key compound in generating the growth of $\mathrm{CVD}$ diamond.

\subsection{Measurement and Characterization of CVD Diamond}

A variety of techniques can be used to characterize CVD diamond films. Measurement and characterization techniques used in this investigation include

1. Scanning electron microscopy and transmission electron microscopy (SEM and TEM), to determine surface morphology, microstructure, and grain size

2. Surface profilometry and atomic force microscopy (AFM), to measure surface roughness and to determine surface morphology

3. Rutherford backscattering (RBS) and elastic recoil spectroscopy (ERS), to determine the composition (including hydrogen)

4. Raman spectroscopy, to characterize the atomic bonding state and quality of diamond

5. X-ray diffraction (XRD), to determine the crystal orientation of diamond

6. Friction measurement, to determine the coefficient of friction and surface properties

Case studies described in the references $(59,60,61)$ focus attention primarily on microwave-plasmaassisted CVD diamond films.

\subsection{Electron Microscopy, Stylus Profilometry, and Atomic Force Microscopy}

Transmission electron microscopy offers image and diffraction modes for specimen observation (63). In the image mode, analysis of transmitted electron images yields information both about atomic structure and about defects present in the material. In the diffraction mode, an electron diffraction pattern is obtained from the specimen area illuminated by the electron beam. The electron diffraction pattern is entirely equivalent to an $\mathrm{x}$-ray diffraction pattern.

Scanning electron microscopy (with energy-dispersive $\mathrm{x}$-ray spectroscopy) is the most useful tool when the researcher needs not only morphological and topographical information about surfaces but also information concerning the composition of near-surface regions of the material. Although diamond is an insulator, it can be studied by using low primary electron beam voltages ( $5 \mathrm{keV}$ or less) if one is willing to compromise image resolution to some extent. If the diamond is coated with a thin conducting film (10 to $20 \mathrm{~nm}$ thick) of carbon, gold, or some other metal, the coated diamond can be studied with an image resolution of 1 to $50 \mathrm{~nm}$. 
TABLE 6.-DEPOSITION CONDITIONS FOR DIAMOND FILMS OF VARIOUS GRAIN SIZES

\begin{tabular}{|c|c|c|c|c|c|c|c|}
\hline \multirow[t]{2}{*}{ Condition } & \multicolumn{7}{|c|}{ Substrate $^{a}$} \\
\hline & Si (100) & Si (100) & $\alpha-S i C$ & $\alpha-\mathrm{SiC}$ & $\alpha_{-}^{-S i C}$ & $\mathrm{Si}_{3} \mathrm{~N}_{4}$ & $\mathrm{Si}_{3} \mathrm{~N}_{4}$ \\
\hline $\begin{array}{l}\text { Deposition } \\
\text { temperature, }{ }^{\circ} \mathrm{C}\end{array}$ & $860 \pm 20$ & $1015 \pm 50$ & $1015 \pm 50$ & $965 \pm 50$ & $860 \pm 20$ & $965 \pm 50$ & $860 \pm 20$ \\
\hline $\begin{array}{l}\text { Gaseous flow rate, } \\
\mathrm{cm}^{3} / \mathrm{min} \text { : } \\
\mathrm{CH}_{4} \\
\mathrm{H}_{2} \\
\mathrm{O}_{2}\end{array}$ & $\begin{array}{r}4 \\
395 \\
1\end{array}$ & $\begin{array}{r}3.5 \\
500 \\
0\end{array}$ & $\begin{array}{r}3.5 \\
500 \\
0\end{array}$ & $\begin{array}{r}3.5 \\
500 \\
0\end{array}$ & $\begin{array}{r}4 \\
395 \\
1\end{array}$ & $\begin{array}{r}3.5 \\
5800 \\
0\end{array}$ & $\begin{array}{r}4 \\
395 \\
1\end{array}$ \\
\hline Pressure, torr & 5 & 40 & 40 & 40 & 5 & 40 & 5 \\
\hline $\begin{array}{l}\text { Microwave power, } \\
\text { W }\end{array}$ & 500 & 100 & 1000 & 1000 & 500 & 1000 & 1500 \\
\hline Deposition time, $\mathrm{hr}$ & 10.5 & 140 & 14 & 22 & 21 & 22 & 521 \\
\hline Thickness, $\mathrm{nm}$ & 1000 & 4200 & 5000 & 8000 & 1000 & 7000 & 800 \\
\hline Grain size, $\mathrm{nm}$ & 20 to 100 & 1100 & 3300 & 1500 & 22 to 100 & 1000 & 22 to 100 \\
\hline $\begin{array}{l}\text { Surface roughness } \\
\mathrm{rms}, \mathrm{nm}\end{array}$ & 15 & 63 & 160 & 92 & 50 & 52 & 35 \\
\hline
\end{tabular}

${ }^{a}$ Scratched with $0.5-\mathrm{mm}$ diamond paste.

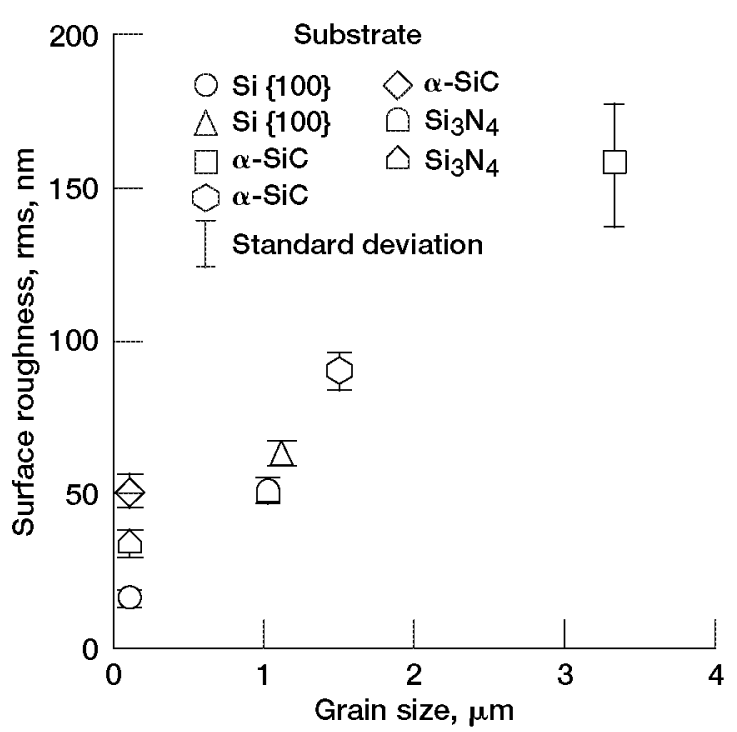

Figure 22.-Surface roughness as function of grain size for diamond films.
The grain size, surface morphology, and surface roughness of a microwave-plasma-assisted CVD diamond film can be controlled by varying the deposition parameters, such as gas-phase chemistry parameters and temperatures (e.g., Table 6). The grain size and surface roughness data were obtained by using TEM and stylus profilometry, respectively. The CVD diamond films referred to in Table 6 can be divided into three groups by grain size: fine, medium, and coarse grain. The grain sizes of the finegrain diamond films were determined from brightand dark-field electron micrographs to be between 20 and $100 \mathrm{~nm}$. The medium- and coarse-grain diamond films have grain sizes estimated at 1000 to $1500 \mathrm{~nm}$ and $3300 \mathrm{~nm}$, respectively. The average surface roughness of the diamond films measured by a surface profilometer increases as the grain size increases, as shown in Fig. 22. Figure 23 shows scanning electron micrographs of fine-, medium-, and coarse-grain diamond films. Triangular crystalline facets typical of diamond are clearly evident on the surfaces of the medium- and coarse-grain films.

In an atomic force microscope a probe tip traverses across a diamond surface and senses the force of interaction between itself and the diamond surface. By monitoring the tip deflection necessary to maintain a constant interacting force, surface topographical data can be obtained on a nanometer scale. Figure 24 shows an AFM image of a chemical-vapor-deposited, fine-grain diamond film on a mirror-polished silicon substrate, along with a histogram and bearing ratio. The CVD diamond surface has a granulated or spherulitic morphology with spherical asperities of different sizes. The surface roughness of the CVD diamond on silicon is $58.8 \mathrm{~nm} \mathrm{rms}$. 

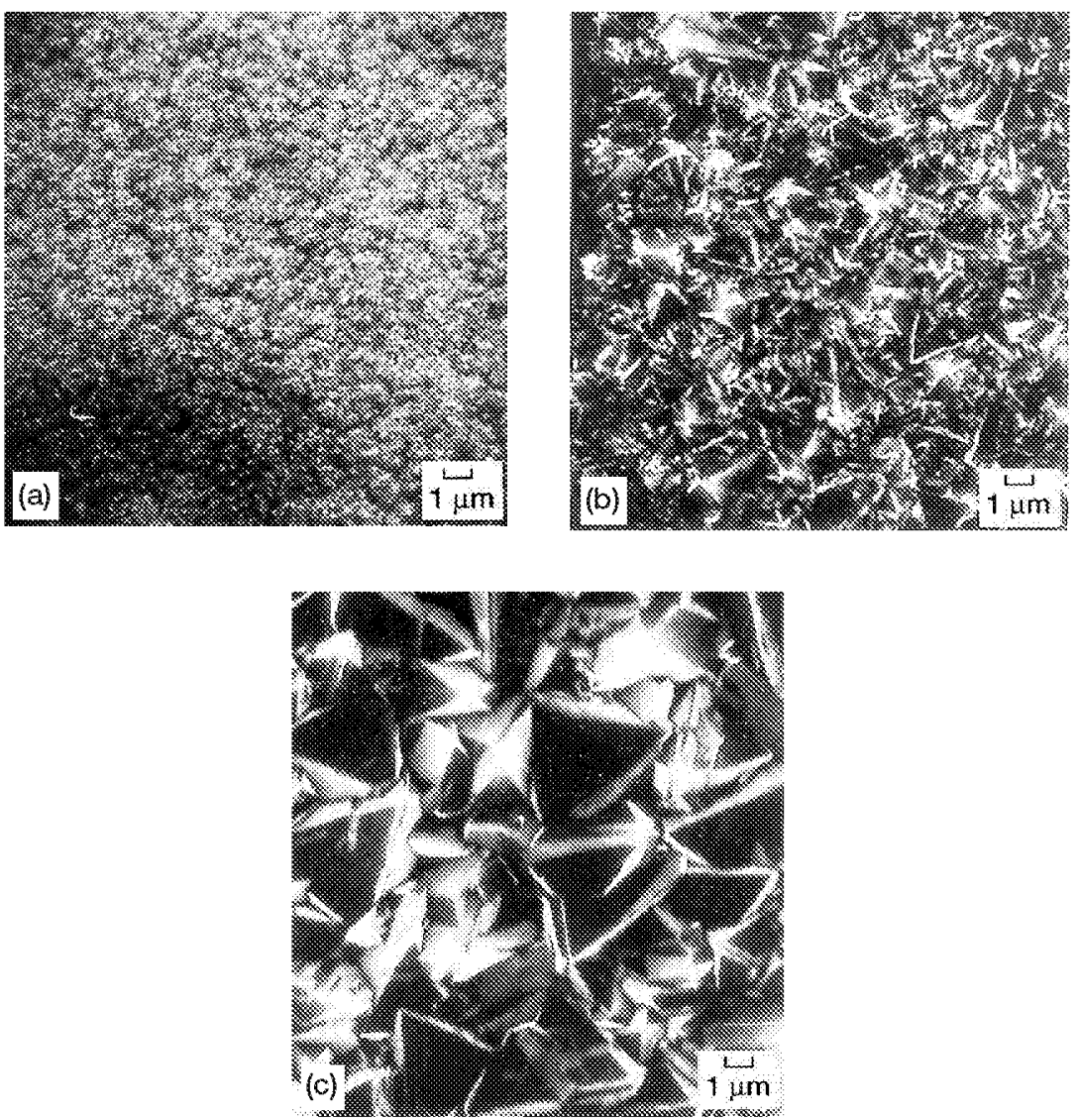

Figure 23. - Scanning electron micrographs of diamond films. (a) Fine-grain (20 to $100 \mathrm{~nm}$ ) diamond film on $\{100\}$ silicon substrate; rms surface roughness, $15 \mathrm{~nm}$. (b) Medium-grain (1100 $\mathrm{nm})$ diamond film on $\{100\}$ silicon substrate; rms surface roughness, $63 \mathrm{~nm}$. (c) Coarse-grain (3300 nm) diamond film on $\{100\} \alpha-S i C$ substrate; ms surface roughness, $160 \mathrm{~nm}$.

\subsection{X-Ray Diffraction and Electron Diffraction}

Although x-ray diffraction (XRD) is not inherently a surface characterization technique, yet it offers unparalleled accuracy in the measurement of atomic spacing (63). XRD was used to determine the structure and crystal orientation of the CVD diamond films (60). Typical x-ray diffraction patterns for the fineand medium-grain diamond films (Fig. 25) show peaks representing only the diamond film and the silicon substrate. Diffraction peaks corresponding to the $\{111\},\{220\},\{311\}$, and $\{400\}$ planes, reflective of diamond, are clearly evident. The intensity ratios $I\{220\} / I\{111\}$ were calculated from the x-ray diffraction patterns for these films and found to be 1.3 and 0.04 , respectively. The powder diffraction pattern of diamond with random crystal orientation (ASTM 6-0675) gives $I\{220\} / I\{111\}=0.27$. Thus, most of the crystallites in the fine-grain diamond film are oriented along the $\langle 110\rangle$ direction, whereas most of the crystallites in the medium-grain diamond films are oriented along the $\langle 111\rangle$ direction. The well-formed triangular facets observed in SEM micrographs of medium- and coarse-grain diamond films confirm the $\langle 111\rangle$ crystal orientation.

Figure 26 presents a TEM selected-area diffraction pattern (SAD), a TEM bright-field micrograph, and a TEM dark-field micrograph of a free-standing, fine-grain CVD diamond film (59). Diffraction rings 

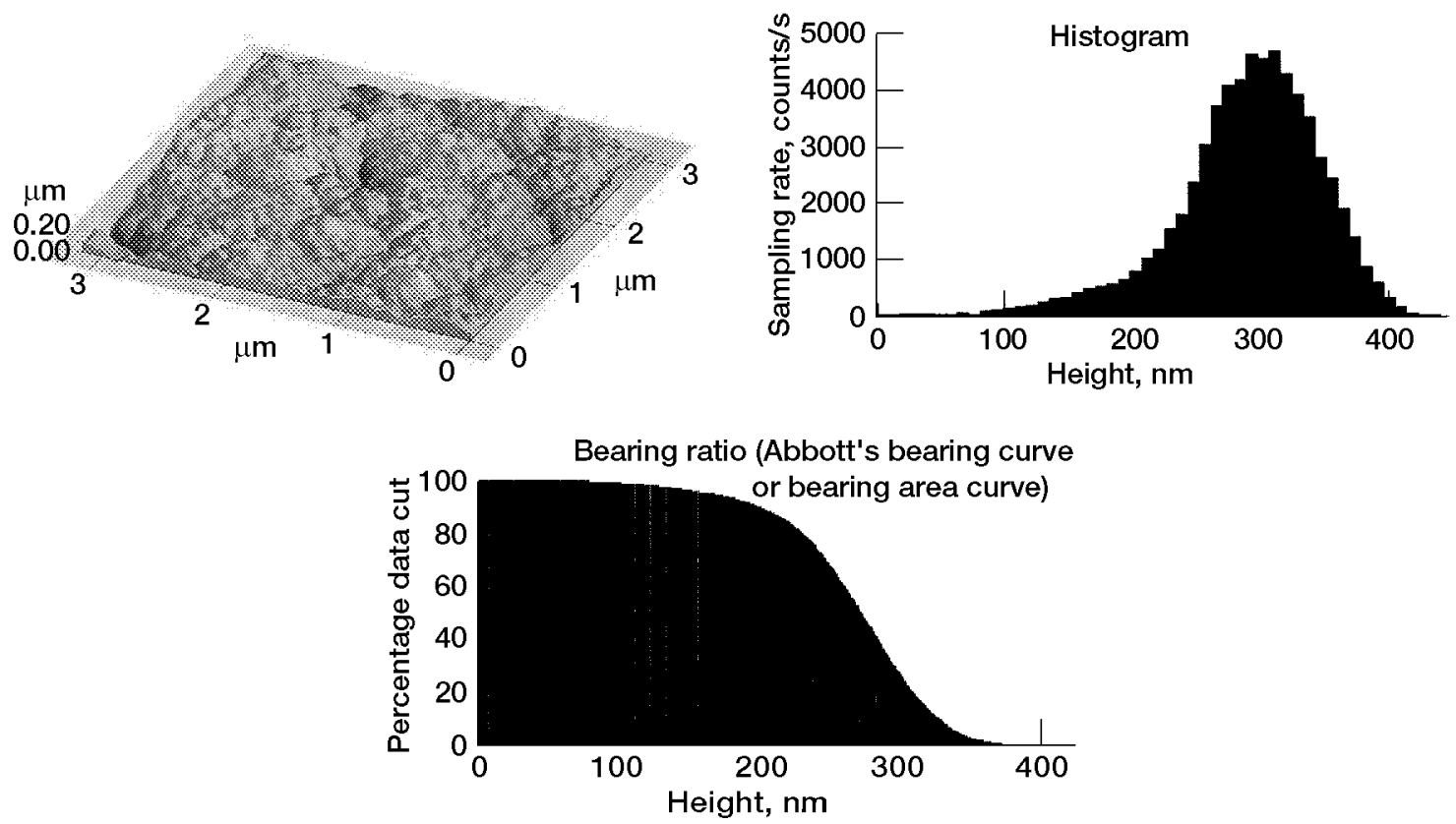

Figure 24.-Atomic force microscopy of chemical-vapor-deposited, fine-grain diamond film on mirrorpolished silicon substrate.
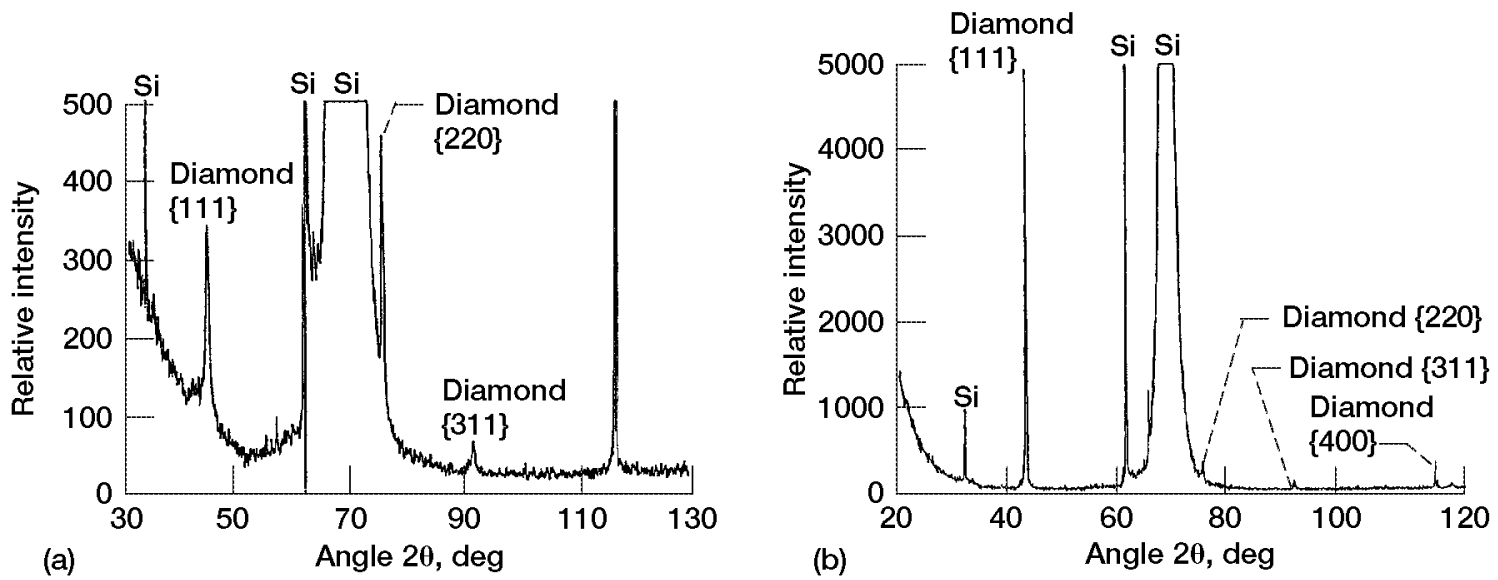

Figure 25. X-ray diffraction patterns of diamond films. (a) Fine-grain $(20$ to $100 \mathrm{~nm}$ ) diamond film on \{100\} silicon substrate. (b) Medium-grain (1100 nm) diamond film on \{100\} silicon substrate.

and dots can be observed in Fig. 26(a). The d spacings of the diffraction rings were calculated by using an aluminum SAD as a calibration standard and were found to match well with the known diamond $d$ spacings. No evidence of nondiamond carbon was found in the SAD. This observation indicates that the nondiamond carbon concentration in the diamond film was extremely small. Careful observation of Fig. 26(b) revealed various nuclei-like regions marked N. Diamond grains are distributed radially outward from these nuclei. A grain boundary is formed where the grains from various nuclei meet. As previously mentioned, the grain sizes of the fine-grain CVD diamond films estimated from the bright- and dark-field micrographs varied from 20 to $100 \mathrm{~nm}$. 

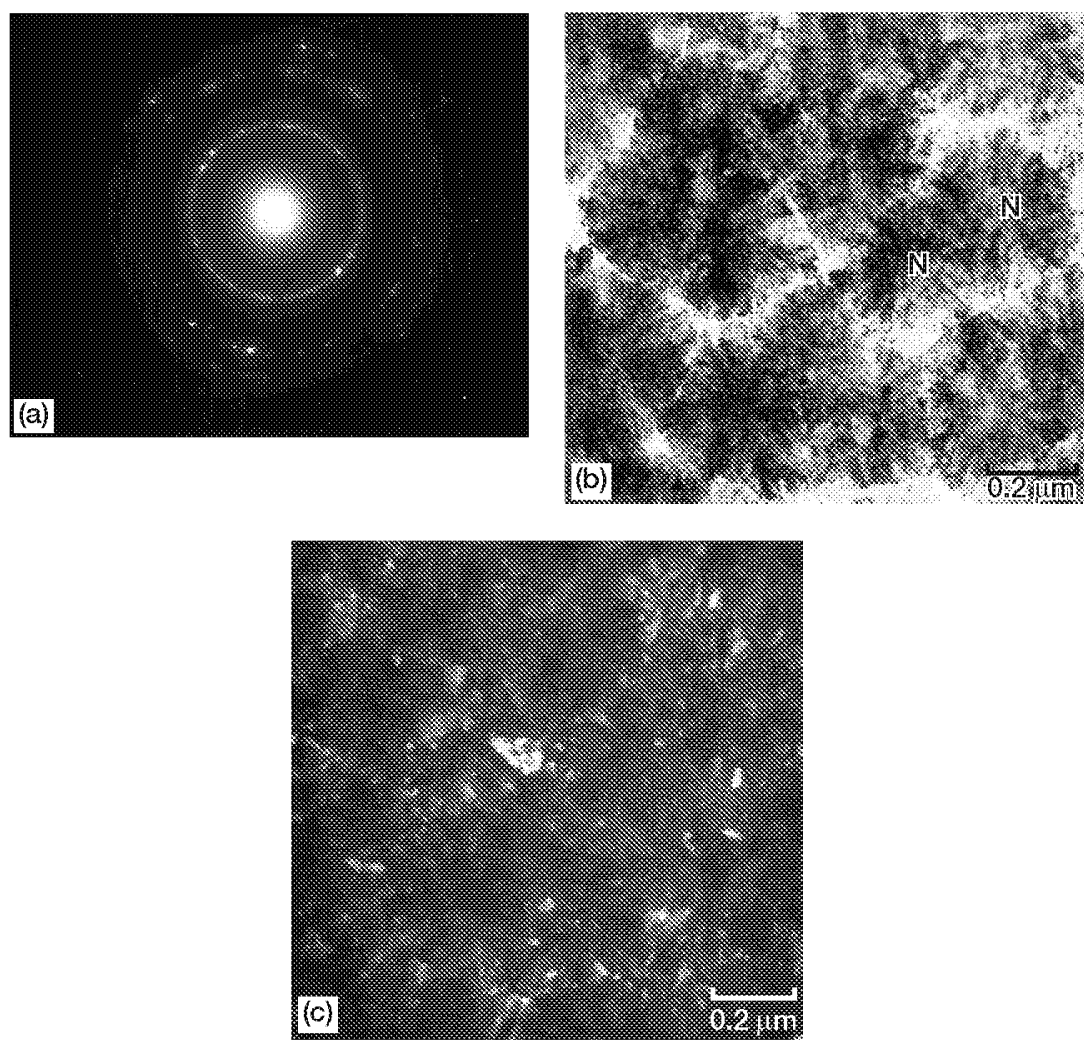

Figure 26.-Free-standing diamond film. (a) Selected-area diffraction pattern. (b) Bright-field TEM. (c) Dark-field TEM.

\subsection{Raman Spectroscopy}

Raman spectroscopy is primarily a structural characterization tool (63). Raman spectra are more sensitive to the lengths, strengths, and arrangement of bonds in a material than to chemical composition. Raman spectra of crystals likewise reflect the details of defects and disorder rather than trace impurities and related chemical imperfections. The laser-optical Raman technique can determine with great confidence the atomic bonding states of the carbon atoms ( $\mathrm{sp}^{2}$ for graphite or $\mathrm{sp}^{3}$ for diamond) from their different vibrational modes (64). Raman spectra result from the inelastic scattering of optical photons by lattice vibration phonons.

Typical Raman spectra of the fine- and medium-grain diamond films (Fig. 27) show one Raman band centered at $1332 \mathrm{~cm}^{-1}$ and one centered around $1530 \mathrm{~cm}^{-1}$. The sharp peak at $1332 \mathrm{~cm}^{-1}$ is characteristic of the $\mathrm{sp}^{3}$ bonding of the diamond form of carbon in the film. The very broad peak centered around $1530 \mathrm{~cm}^{-1}$ is attributed to the $\mathrm{sp}^{2}$ bonding of the nondiamond forms of carbon (graphite and other carbon) $(65,66,67)$.

More $\mathrm{sp}^{3}$-bonded (diamond) carbon is produced in larger grained CVD diamond films (e.g., Fig. 27(b)) than in fine-grain films, as is evident from the relative intensities of the diamond and nondiamond carbon Raman bands (60). However, the ratio of the intensities of the Raman responses at $1332 \mathrm{~cm}^{-1}$ and centered around $1530 \mathrm{~cm}^{-1}$ does not indicate the ratio of diamond to nondiamond carbon present in a particular film, since the Raman technique is approximately 50 times more sensitive to $\mathrm{sp}^{2}$-bonded (nondiamond) carbon than to $\mathrm{sp}^{3}$-bonded (diamond) carbon (66). Thus, the peak centered around $1530 \mathrm{~cm}^{-1}$ for each film represents a much smaller amount of nondiamond carbon in these diamond films than appears at first glance. 

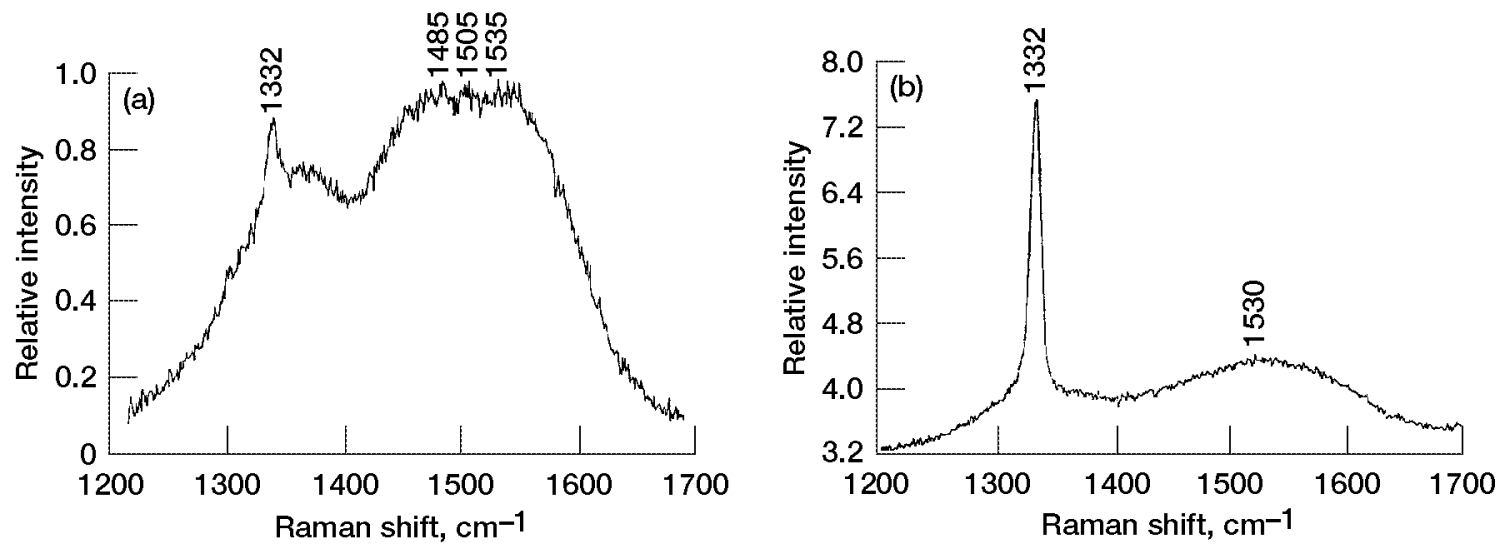

Figure 27.-Raman spectra of diamond films. (a) Fine grain (20 to $100 \mathrm{~nm}$ ) diamond film on $\{100\}$ silicon substrate. (b) Medium-grain (1100 nm) diamond film on $\{100\}$ silicon substrate.

\subsection{Rutherford Backscattering Spectroscopy and Elastic Recoil Spectroscopy}

Rutherford backscattering spectroscopy (RBS) is a nondestructive, quantitative depth profiling of thin film compositions and structures, crystallinity, dopants, and impurities (63). Elastic recoil spectroscopy (ERS, hydrogen forward scattering or proton recoil detection) is the simplest ion beam technique for hydrogen profiling and determining hydrogen concentrations in thin films. In combination with RBS analysis of the same sample, ERS provides concentration profiles and complete compositional analysis of the near-surface regions of the sample material.

Figures 28 and 29 present RBS and ERS spectra, respectively, of a fine-grain CVD diamond film (59). Besides carbon from the diamond film and silicon from the silicon substrate, no other elements were observed in the RBS spectrum. From both spectra it was estimated that the fine-grain diamond film consisted of 97.5 at.\% carbon and 2.5 at.\% hydrogen. (In contrast, the medium-grain diamond films contained less than 1 at.\% hydrogen (60).) It was also demonstrated that both carbon and hydrogen are uniformly distributed in the fine-grain film from the top of the surface to the silicon substrate.

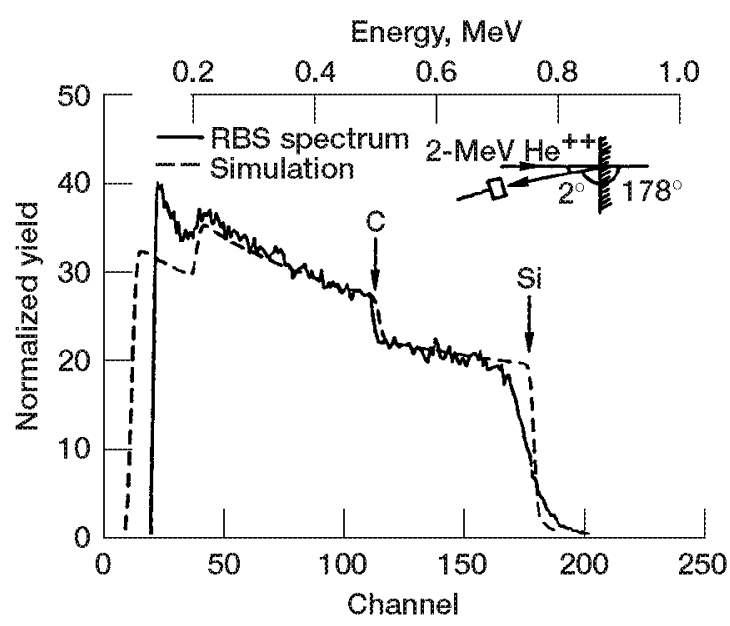

Figure 28.-Rutherford backscattering spectrum of fine-grain diamond film on silicon substrate. (Simulation curve was calculated by using the computer code RUMP.)

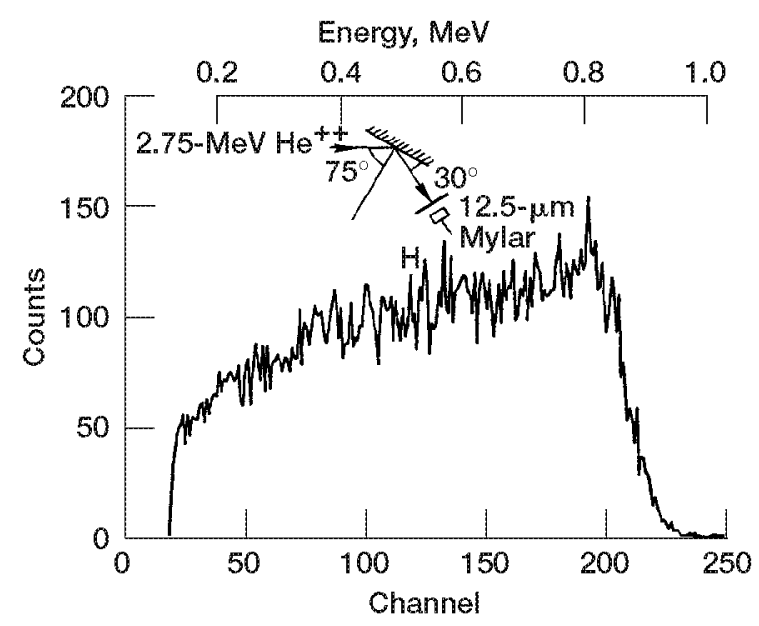

Figure 29.-Elastic recoil spectrum of fine-grain diamond film on silicon substrate. 
RBS analytical results can also be used to determine diamond film thickness. Figure 28 presents a simulated RBS spectrum of a diamond film with a carbon-to-hydrogen ratio $(\mathrm{C} / \mathrm{H})$ of 97.5/2.5 obtained by using the RUMP computer code (68). In the computer program the film thickness of the diamond film is taken as a variable. This thickness was obtained from the close match between the observed and simulated RBS, as shown in Fig. 28 , and is $1.5 \mu \mathrm{m}$ at the center of the substrate. The deposition rate was estimated to be $0.14 \mu \mathrm{m} / \mathrm{hr}$.

\subsection{Friction Measurement}

The classical Bowden and Tabor model for sliding friction of materials in contact, in its simple form, assumes that the friction force arises from two contributing sources (69). First, an adhesion force is developed at the real area of contact between the surfaces, arising from the attractive forces between the contacting surfaces. Second, a deformation force is needed to plow or cut the asperities of the harder surface through the softer. The resultant friction force is the sum of the two contributing sources: friction due to adhesion and friction due to deformation and/or fracture. When a smooth flat is brought into contact with a smooth spherical surface of the same or softer material, the plowing or cutting contribution in friction can be neglected. In this situation, as is well known, diamond is one of the slipperiest materials and is similar to polytetrafluoroethylene in air. The coefficient of friction in air is between 0.01 and 0.05 .

The friction and wear properties of CVD diamond films are similar to those of natural and synthetic diamond. The coefficient of friction and wear resistance of CVD diamond are generally superior in the atmosphere. However, the environment to which a CVD diamond film is exposed can markedly affect its friction and wear behavior, giving it a Jekyll-and-Hyde character (60). For example, the coefficient of friction in ultrahigh vacuum is greater than 0.4 .

6.8.1 Humid Air and Dry Nitrogen Environments.-When the fine-, medium-, and coarse-grain CVD diamond films characterized in previous sections were brought into contact with a natural diamond pin in reciprocating sliding motion in air and in nitrogen, the coefficients of friction varied as the pin traveled back and forth (reciprocating motion), retracing its tracks on the diamond films (Fig. 30).

Both in humid air, at a relative humidity of $40 \%$, and in dry nitrogen, abrasion occurred and dominated the friction and wear behavior. The bulk natural diamond pin tended to dig into the surface of diamond films during sliding and to produce a wear track (groove). SEM observations of the diamond films indicated that small fragments chipped off the surfaces. When abrasive interactions between the diamond pin surface and the initially sharp tips of asperities on the diamond film surfaces were strong, the friction was high (points A in Fig. 30). The surface roughness of diamond films can appreciably influence their initial friction (i.e., the greater the initial surface roughness, the higher the initial coefficient of friction, Fig. 31). Similar frictional results have also been found by other workers on single-crystal diamonds (70) and on diamond coatings $(71,72,73)$.

As sliding continued and the pin passed repeatedly over the same track, the coefficient of friction was appreciably affected by the wear on the diamond films (Fig. 32) (i.e., a blunting of the asperity tips). When repeated sliding produced a smooth groove or a groove with blunted asperities on the diamond surface, the coefficient of friction (only due to adhesion) was low, and the initial surface roughness effect became negligible. Therefore, the equilibrium coefficient of friction was independent of the initial surface roughness of the diamond film (Fig. 31(b)).

The generally accepted wear mechanism for diamonds is that of small fragments chipping off the surface $(60,74)$. This mechanism is in agreement with the wear of diamond films. The wear rate is dependent on the initial surface roughness of the diamond films (Fig. 33), increasing markedly with an increase in initial surface roughness. The wear rates of the diamond films in humid air and in dry nitrogen are comparable to the wear rates of single-crystal diamonds and diamond films investigated by other workers $(61,71,75)$. 

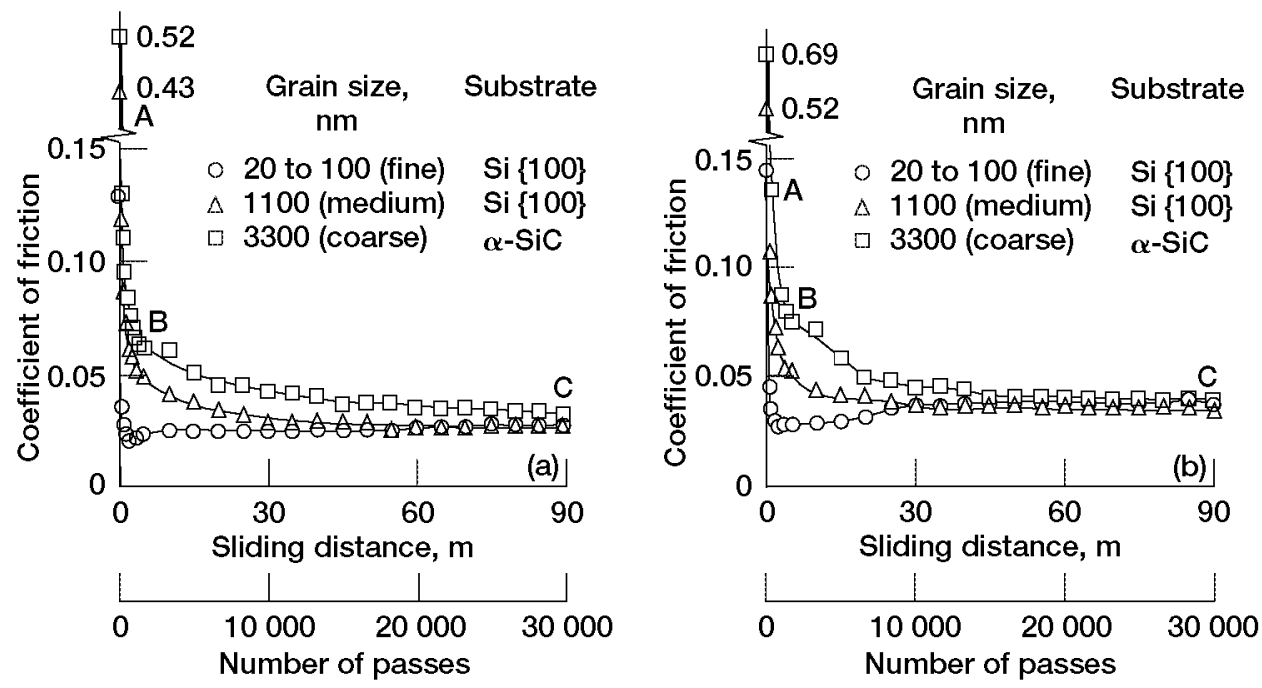

Figure 30.-Coefficient of friction as function of number of passes of bulk diamond pin in contact with fine-, medium-, and coarse-grain diamond films (a) in humid air (approx. $40 \%$ relative humidity) and (b) in dry nitrogen.

6.8.2 Ultrahigh Vacuum Environment.-When the fine-, medium-, and coarse-grain diamond films were brought into contact with a natural diamond pin in unidirectional pin-on-disk sliding motion in vacuum, the coefficients of friction were high and varied with the number of passes (60). In vacuum, as in humid air and in dry nitrogen, the bulk natural diamond pin dug into the surfaces of the diamond films during sliding and produced a wear track (groove, Fig. 34). The groove surface was generally smoother than the original surface of the diamond films. Further analysis of the grooves by scanning electron microscopy revealed that the tips of the diamond coating asperities were worn smooth and that the gaps between asperities were filled by debris.

The coefficient of friction increased with an increase in the number of passes (Fig. 35), just the opposite of what occurred in humid air and in dry nitrogen. Further, the initial surface roughness of the diamond film had no effect on friction. These results led us to ask the following questions: What factors determine friction behavior? Have dangling bonds been exposed during sliding and played a role in the friction behavior? Which is more important for diamond surfaces in vacuum, abrasion or adhesion?

Removing some of the contaminant surface film from the contact area of diamond films by sliding action resulted in stronger interfacial adhesion between the diamond pin and the diamond films and raised the coefficient of friction, as shown in Fig. 35. A contaminant surface film may be removed by repeatedly sliding the diamond pin over the same track in ultrahigh vacuum (61).

The friction results shown in Fig. 35 agree with other researchers' results for single-crystal diamond rubbing against diamond and for CVD diamond sliding against CVD diamond in vacuum $(76,77)$. Bowden and Hanwell (77) observed an initial coefficient of friction of 0.1 for diamond on diamond; within several hundred passes, however, the coefficient of friction rose rapidly to 0.9 and remained constant. Dugger, Peebles, and Pope (78) also found that the coefficient of friction increased to 0.47 when CVD diamond slid against itself in vacuum $(<0.6 \mathrm{mPa})$. In both cases the increase in friction was attributed to cleaning the adsorbed contaminants from the surface by rubbing or sliding in vacuum at room temperature.

When sliding continues, the wear dulls the tips of the diamond grains and increases the contact area in the wear track, thereby causing an increase in friction. The increase in equilibrium friction that results from cleaning off the contaminant surface film by sliding and from increasing the contact area is greater than the corresponding decrease in abrasion and friction that results from blunting the tips of surface 

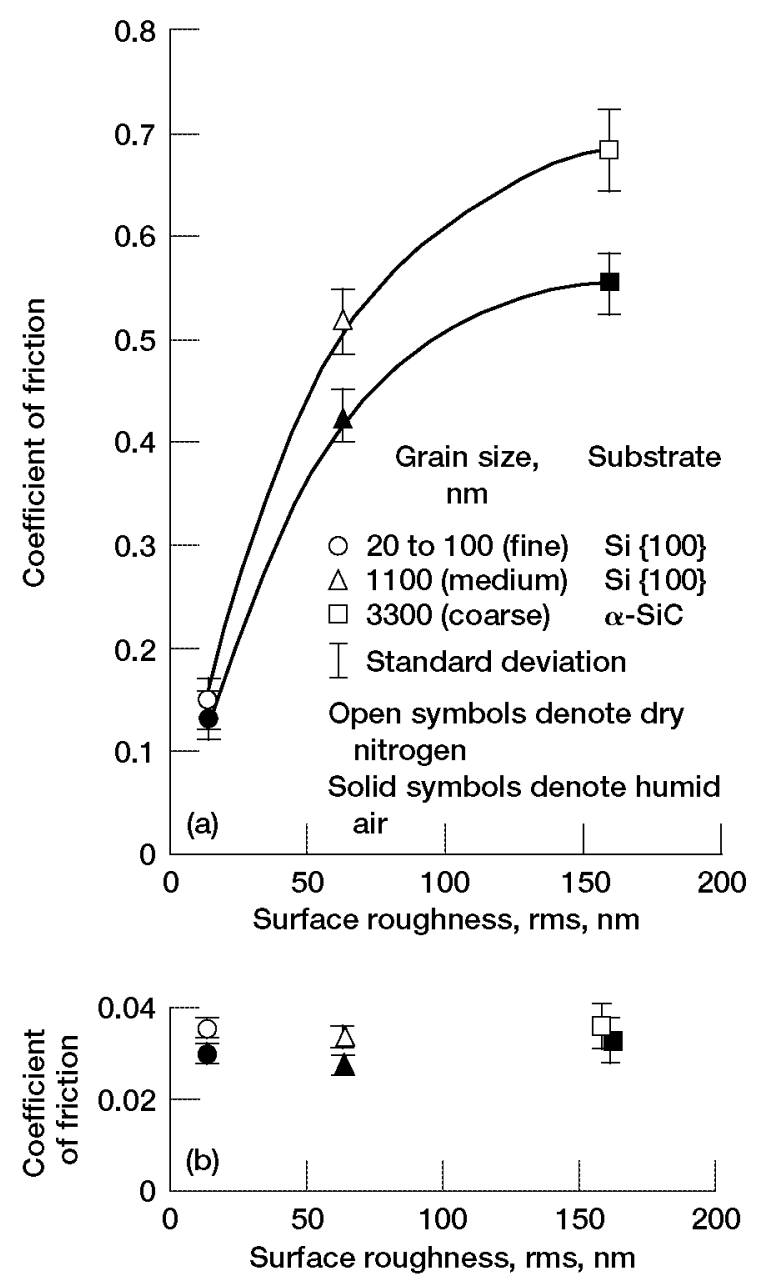

Figure 31.-Coefficient of friction as function of initial surface roughness of diamond films in humid air (approx. 40\% relative humidity) and in dry nitrogen. (a) Initial coefficients of friction. (b) Equilibrium coefficients of friction.

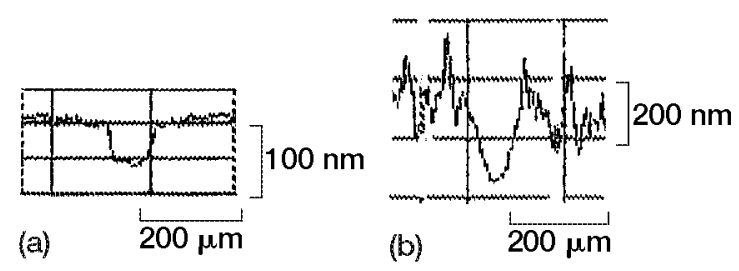

(a)

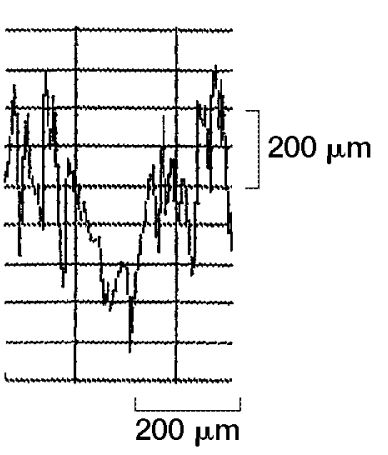

Figure 32-Wear tracks (grooves) on diamond films after 30000 passes of bulk diamond pins in dry nitrogen. (a) Fine-grain (20 to $100 \mathrm{~nm}$ ) diamond film; rms surface roughness, $15 \mathrm{~nm}$. (b) Mediumgrain (1100 $\mathrm{nm}$ ) diamond film; rms surface roughness, $63 \mathrm{~nm}$. (c) Coarse-grain (3300 nm) diamond film; rms surface roughness, $160 \mathrm{~nm}$.

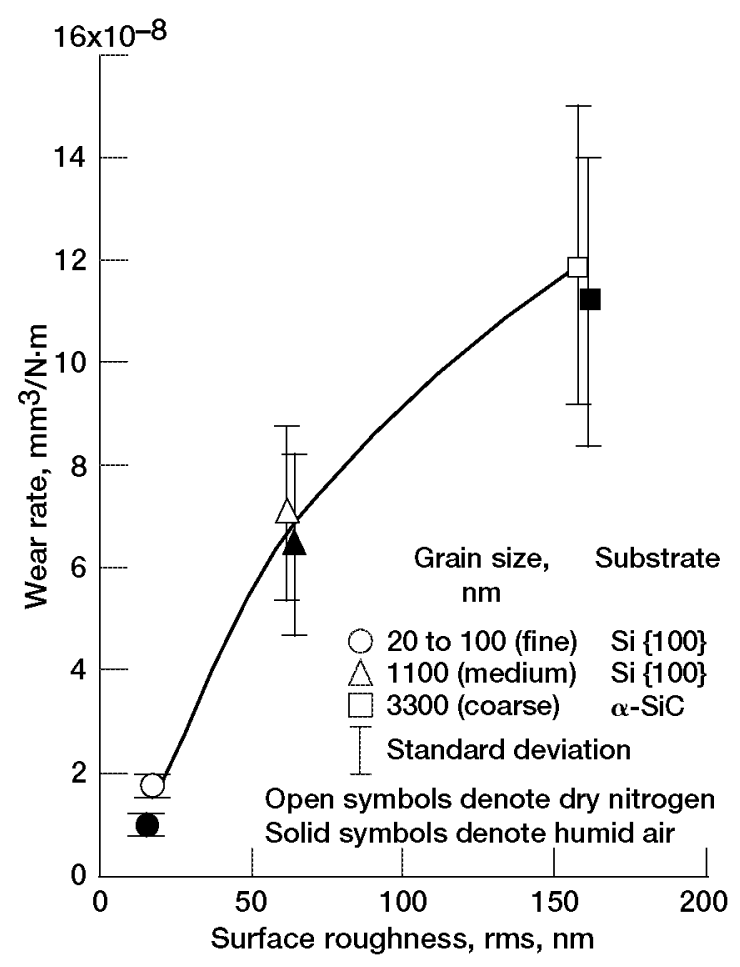

Figure 33. - Wear rate as function of diamond surface roughness for diamond films in humid air and dry nitrogen. 


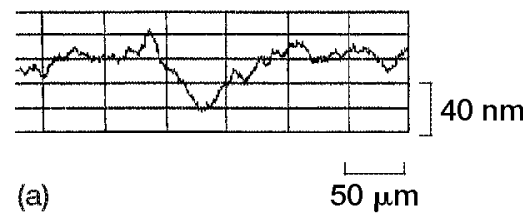

(a)

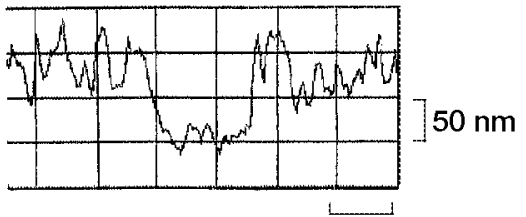

(b)
$50 \mu \mathrm{m}$

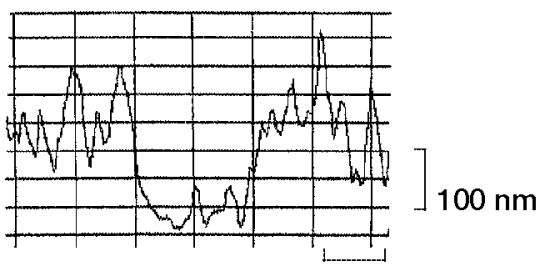

(c)

$50 \mu \mathrm{m}$

Figure 34. -Wear tracks (grooves) on diamond films after 100 passes of bulk diamond pin in ultrahigh vacuum. (a) Fine-grain (20 to $100 \mathrm{~nm}$ ) diamond film on silicon substrate; rms surface roughness, $15 \mathrm{~mm}$. (b) Medium=grain $(1000 \mathrm{~nm})$ diamond film on silicon nitride substrate; rms surface roughness, $52 \mathrm{~nm}$. (c) Coarse-grain (1500 nm) diamond film on $\alpha$-SiC substrate; rms surface roughness, $92 \mathrm{~nm}$.

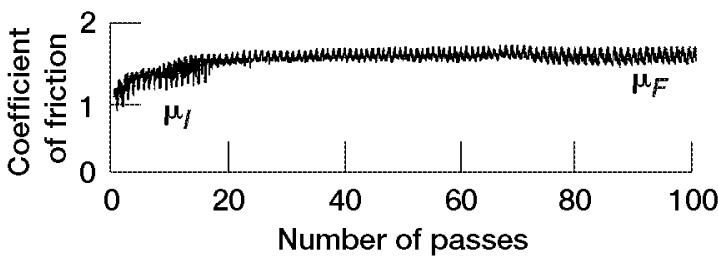

Figure 35,-Typical friction trace for bulk diamond pin in contact with diamond film on $\alpha$-SiC substrate in ultrahigh vacuum (initial coefficient of friction, $\mu_{f}$; equilibrium coefficient of friction, $\mu_{F}$ ).

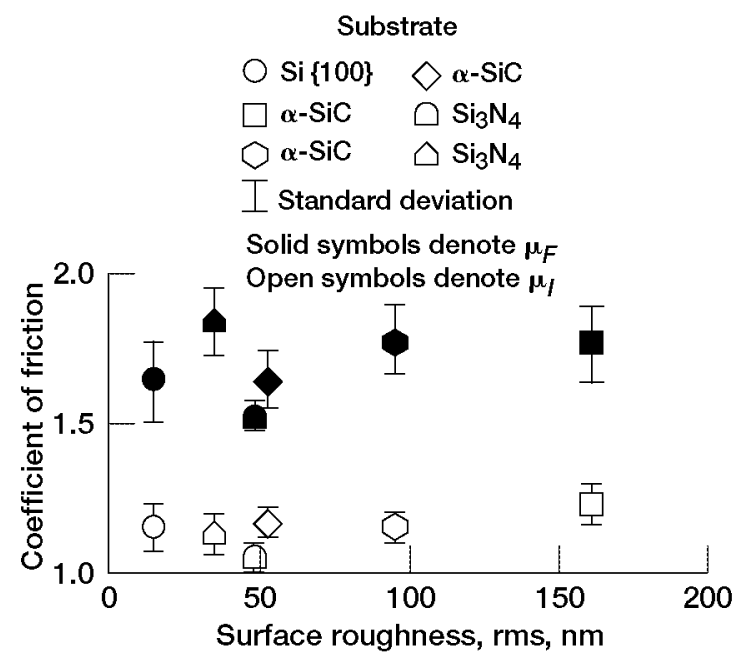

Figure 36 .-Inital $\mu_{1}$ and equilibrium $\mu_{F}$ coefficient of friction as function of initial surface roughness for diamond films in ultrahigh vacuum.

asperities. This relationship is brought out clearly in Fig. 36; here the equilibrium coefficients of friction (1.5 to 1.8) are greater than the initial coefficients of friction (1.1 to 1.3) regardless of the initial surface roughness of the diamond films. In vacuum, therefore, the friction arises primarily from adhesion between the sliding surfaces of the diamond pin and the diamond films.

The wear rates of the diamond films in ultrahigh vacuum (Fig. 37) depended on the initial surface roughness of the diamond films, generally increasing with an increase in initial surface roughness. The wear rates of the diamond films in ultrahigh vacuum were considerably higher (by a factor of 3000) than those of the diamond films in humid air or in dry nitrogen (Fig. 33). Obviously, under these vacuum conditions adhesion between the sliding surfaces of the diamond pin and the diamond films played an important role in the higher wear process. 


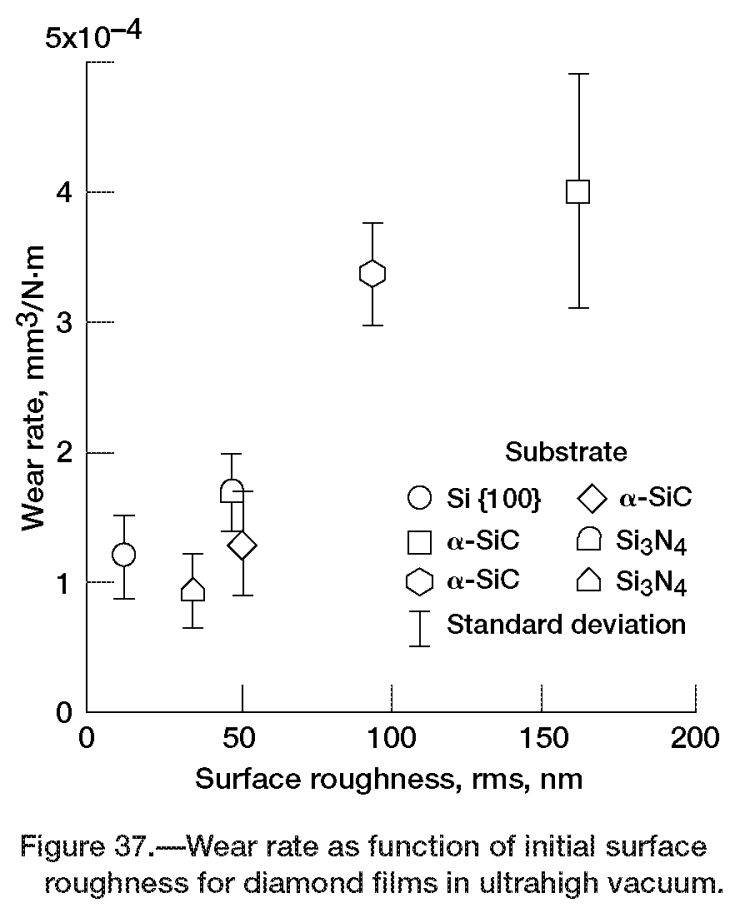

Thus, under vacuum conditions it is adhesion between the sliding surfaces of the diamond pin and the diamond films (due to the highly clean state) and the possible presence of dangling bonds that play a significant role in the friction and wear process. The surface roughness of the diamond films does not have much influence on the friction of diamond films in ultrahigh vacuum.

\subsection{Summary of Remarks}

The technical application and utility of CVD diamond in specific component systems and environments can be achieved if the deposition parameters have been optimized to achieve the desired quality, properties, and durability. To understand the benefits provided by the deposition parameters, and ultimately to provide even better deposition parameters and greater film and coating performance, researchers must use a variety of measurement and diagnostic techniques to investigate the physical, chemical, material, and structural changes in films and coatings produced at different deposition parameters.

The use of measurement and characterization techniques, including friction measurements, was highlighted in the important case study of microwave-plasma-assisted, chemical-vapor-deposited diamond films. In this study a combination of measurement and characterization techniques was successfully applied to correlate the coating deposition parameters with the resultant diamond film composition, crystallinity, grain size, surface roughness, and coefficient of friction. These techniques have contributed significantly to the understanding of the quality and properties of diamond films and their surfaces. 


\section{Concluding Remarks}

To understand the benefits that surface modifications provide, and ultimately to extend these benefits, it is necessary to study the physical, mechanical, and chemical changes they cause. A wide variety of surface characterization techniques is available for assessing the physical, mechanical, and chemical properties of surfaces. Each measurement and characterization technique provides unique information. It should be possible to coordinate the different pieces of information provided by these measurement and diagnostic techniques into a coherent self-consistent description of the surface and bulk properties.

\section{References}

1. K Miyoshi, YW Chung. Surface Diagnostics in Tribology: Fundamental Principles and Applications. River Edge, NJ: World Scientific Publishing Co., 1993.

2. K Holmberg, A Matthews. Coatings Tribology: Properties, Techniques and Applications in Surface Engineering. Tribology Series 28, D Dowson, ed. Amsterdam: Elsevier, 1994.

3. B Bunshah et al., eds. Deposition Technologies for Films and Coatings: Developments and Applications. Park Ridge, NJ: Noyes Publications, 1982.

4. TFJ Quinn. Physical Analysis for Tribology. Cambridge, U.K.: Cambridge University Press, 1991.

5. DH Buckley. Surface Effects in Adhesion, Friction, Wear, and Lubrication. Tribology Series 5. Amsterdam: Elsevier, 1981.

6. WA Glaeser, ed. Characterization of Tribological Materials. Stoneham, MA: ButterworthHeinemann and Manning, 1993.

7. CR Brundle, CA Evans, Jr., S Wilson, eds. Encyclopedia of Materials Characterization. Stoneham, MA: Butterworth-Heinemann and Manning, 1992.

8. DM Brewis, ed. Surface Analysis and Pretreatment of Plastics and Metals. New York: Macmillan, 1982.

9. D Briggs, MP Seah. Practical Surface Analysis: By Auger and X-Ray Photo-Electron Spectroscopy, Vol. 1. New York: Wiley, 1983.

10. T Tsukizoe. Precision Metrology. Tokyo, Japan: Yokkendo Publishing, 1970, pp. 180-199.

11. JBP Williamson. The shape of surfaces. In: ER Booser, ed., CRC Handbook of Lubrication, Vol. II. Boca Raton, FL: CRC Press Inc., 1984, pp. 3-16.

12. YW Chung. Characterization of topography of engineering surfaces. In: K Miyoshi, YW Chung, eds. Surface Diagnostics in Tribology: Fundamental Principles and Applications. River Edge, NJ: World Scientific Publishing, 1993, pp. 33-46.

13. PJ Caber, SJ Martinek, RJ Niemann. A new interferometric profiler for smooth and rough surfaces. WYKO Technical Bulletin 1993-27A, WYKO, Tucson, AZ, 1993, pp. 1-14.

14. V Chandrasekaran, YI Yoon, DW Hoeppner. Analysis of fretting damage using confocal microscope. In: DW Hoeppner, V Chandrasekaran, CB Elliott III, eds. Fretting Fatigue: Current Technology and Practices. ASTM STP 1367, West Conshohocken, PA: American Society for Testing and Materials, 2000, pp. 337-351.

15. T Wilson. Confocal Microscopy. San Diego, CA: Academic Press, Inc., 1990.

16. RS Howland, J Okagaki, L Mitobe. How to buy a scanning probe microscope, Sunnyvale, CA: Park Scientific Instruments, 1993.

17. W Weiler. Are hardness values quantities or characteristics? In: JH Westbrook, H Conrad, eds., The Science of Hardness Testing and Its Research Applications. Metals Park, OH: American Society for Metals, 1973, pp. 16-20.

18. JJ Gilman. Hardness - a strength microprobe. In: JH Westbrook, H Conrad, eds., The Science of Hardness Testing and Its Research Applications. Metals Park, OH: American Society for Metals, 1973, pp. 51-74. 
19. MC Shaw. The fundamental basis of the hardness test. In: JH Westbrook, H Conrad, eds., The Science of Hardness Testing and Its Research Applications. Metals Park, OH: American Society for Metals, 1973, pp. 1-15.

20. D Tabor. Hardness of Metals. Oxford, U.K.: Clarendon Press, 1951.

21. SG Corcoran. Nanoindentation of Tribological Coatings on Steel. Minneapolis, MN: Hysitron Incorporated, 1997. Also, http://www.hysitron.com.

22. Hardness testing (reference tables). In: ASM Metals Handbook, 8th ed., Vol. 11, Nondestructive Testing and Quality Control. Metals Park, OH: ASM International (formerly American Society for Metals), 1976, pp. 1-20, 425-427.

23. G Totten, MAH Howes (eds.). Steel Heat Treating Handbook. New York: Marcel Dekker, 1999, pp. 1044-1049, 1171-1172.

24. CA Moyer. Appendix 1: Material hardness tables, tests, and data. In: ER Booser, ed., Tribology Data Handbook. Boca Raton, FL: CRC Press, Inc., 1997, pp. 1049-1062.

25. CA Brookes. Indentation hardness. In: JE Field, ed., The Properties of Diamond. New York: Academic Press, 1979, pp. 384-402.

26. K Miyoshi, DH Buckley. Ceramic wear in indentation and sliding contact. ASLE Trans 28, 3:296$302,1985$.

27. GC Rybicki, P Pirouz. Indentation plasticity and fracture in silicon. NASA TP-2863, Washington, DC: National Aeronautics and Space Administration, 1988.

28. H Ishigaki, K Miyoshi, DH Buckley. Influence of corrosive solutions on microhardness and chemistry of magnesium oxide \{001\} surface. NASA TP-2040, Washington, DC: National Aeronautics and Space Administration, 1982.

29. JL Henshall, CA Brookes. Measurement of $\mathrm{K}_{\mathrm{IC}}$ in single crystal $\mathrm{SiC}$ using the indentation method, J Mater Sci Lett 4, 6:783-786, 1985.

30. S Palmqvist. Rissbildungsarbeit bei Vickers-Eindruecken als Mass fuer die Zaehigkeit von Hartmetallen. Arch. Eisenhuettenwesen 33, 9:629-634, 1962.

31. K Niihara. A fracture mechanics analysis of indentation-induced Palmqvist crack in ceramics. J Mater Sci Lett 2, 5:221-223, 1983.

32. AG Evans, EA Charles. Fracture toughness determination by indentation. J Am Ceram Soc 59, 7-8:371-372, 1976.

33. B Lawn, D Marshall. Hardness, toughness, and brittleness indentation analysis. J Am Ceram Soc 62, 7-8:347-350, 1975 .

34. G Antis et al. A critical evaluation of indentation techniques for measuring fracture toughness. I-Direct crack measurements. J Am Ceram Soc 64, 9:533-538, 1981.

35. PTB Shaffer. Effect of crystal orientation on hardness of silicon carbide. J Am Ceram Soc 47, 9:466, 1964.

36. OO Adewoye, GR Sawyer, JW Edington, TF Page. Structural studies of surface deformation in $\mathrm{MgO}, \mathrm{SiC}$, and Si3N4. Annual Technical Report AD-A008993, Cambridge, UK: Cambridge University Press, 1974.

37. BR Lawn, TR Wilshaw. Fracture of Brittle Solids. Cambridge, London, UK: Cambridge University Press, 1975.

38. N Ikawa, S Shimada, T Ono. Microstrength of diamond. Technology Reports of Osaka University 26, 1298:245-254, 1976.

39. N Ikawa, S Shimada. Microstrength measurement of brittle materials. Technology Reports of Osaka University 31, 1622:315-323, 1981.

40. S Bhagavantam, J Bhimasenachar. Elastic constants of diamond. Proc R Soc (London) Ser A 187:381-384, 1946.

41. RM Chrenko, HM Strong. Physical properties of diamond. General Electric Technical Information Series No. 75CRD089, 1975, pp. 1-45.

42. JA Williams. Analytical models of scratch hardness. Tribology Int 29, 8:675-694, 1996. 
43. HE Hintermann. Adhesion, friction, and wear of thin hard coatings. Wear 100:381-397, 1984.

44. HE Hintermann. Surface treatments. In: RK Viswanadham, DJ Rowcliffe, J Gorland, eds. Science of Hard Materials. New York: Plenum Press, 1983, pp. 357-394.

45. K Miyoshi. Fundamental tribological properties of ion-beam-deposited boron nitride thin films. In: Materials Science Forum, Vols. 54\&55, 1990, pp. 375-397.

46. P Benjamin, C Weaver. Measurement of adhesion of thin films. Proc R Soc (London) Ser A 254:163-176, 1960.

47. J Ahn, KL Mittal, RH MacQueen. Hardness and adhesion of filmed structures as determined by scratch technique. In: KL Mittal, ed. Adhesion Measurements of Thin Films, Thick Films, and Bulk Coatings. ASTM STP 640, 1978, pp. 134-157.

48. RP Steijn. On the wear of sapphire. J Appl Phys 32, 10:1951-1958, 1961.

49. K Tanaka, K Miyoshi, Y Miyao, T Murayama. Friction and deformation of Mn-Zn ferrite single crystals. In: T Sakurai, ed. Proceedings of the JSLE-ASLE International Lubrication Conference. Amsterdam: Elsevier Scientific Publishing Co., 1976.

50. K Miyoshi, DH Buckley. Friction, deformation, and fracture of single-crystal silicon carbide. ASLE Trans 22:79-90, 1979.

51. JT Norton, GT Cameron, Sr., eds. Electron optical and x-ray instrumentation for research, product assurance and quality control. Amray Technical Bulletins, Vol. 2, No. 1, Amray, Inc., Bedford, MA, Jan. 1986.

52. YW Chung, HS Cheng. Advances in Engineering Tribology. STLE SP-31, Park Ridge, IL: Society of Tribologists and Lubrication Engineers, 1991.

53. YW Chung, AM Homola, GB Street, eds. Surface Science Investigations in Tribology: Experimental Approaches. ACS Symposium Series 485, Washington, DC: American Chemical Society, 1992.

54. AP Molloy, AM Dionne, eds. Wear and Superhard Coatings 1998. Gorham, ME: Gorham Advanced Materials, Inc., 1998.

55. K Miyoshi et al. Tribological characteristics and applications of superhard coatings: CVD diamond, DLC, and c-BN. NASA/TM-1999-209189, Washington, DC: National Aeronautics and Space Administration, 1999.

56. K Miyoshi. Aerospace mechanisms and tribology technology: case studies. NASA/TM-1999107249, Washington, DC: National Aeronautics and Space Administration, 1999.

57. HO Pierson. Handbook of Carbon, Graphite, Diamond, and Fullerenes. Park Ridge, NJ: Noyes Publications, 1993.

58. K Miyoshi, Chemical-vapor-deposited diamond films. NASA/TM-1999-107249, Washington, DC: National Aeronautics and Space Administration, 1999.

59. RLC Wu, AK Rai, A Garscadden, P Kee, HD Desai, K Miyoshi. Synthesis and characterization of fine grain diamond films. J Appl Phys 72, 1:110-116, 1992.

60. K Miyoshi. Friction and wear of plasma-deposited diamond films. J Appl Phys 74, 7: 4446$4454,1993$.

61. K Miyoshi, RLC Wu, A Garscadden. Friction and wear of diamond and diamond-like carbon coatings. Surf Coat Technol 54/55:428-434,1992.

62. H Windischmann. Stress issues in wafer-scale CVD diamond fabrication. In: Book of Abstracts. New York: American Vacuum Society, 1998, p. 124.

63. CR Brundle, CA Evans, Jr., S Wilson. Encyclopedia of Materials Characterization. Boston, MA: Butterworth-Heinemann, 1992.

64. RJ Nemanich, JT Glass, G Lucovsky, RE Shroder. Raman scattering characterization of carbon bonding in diamond and diamondlike thin films. J Vac Sci Technol A 6, 3:1783-1787, 1988.

65. JJ Cheng, TD Mautei, R Vuppulahadium, HE Jackson. Effects of oxygen and pressure on diamond synthesis in a magnetoactive microwave discharge. J Appl Phys 71, 6: 2918-2923, 1992. 
66. K Kobashi, K Nishinura, V Kawate, T Horiuchi. Synthesis of diamonds by use of microwave plasma chemical vapor deposition: morphology and growth of diamond film. Phys Rev. B38, 6:4067$4084,1988$.

67. N Wada, SA Solin. Raman efficiency measurements of graphite (and Si and Ge). Physica B\&C 105: 353-356, 1981.

68. LR Doolittle. Algorithms for the rapid simulation of Rutherford backscattering spectra. Nucl Instrum Meth B9:344-351, 1985.

69. FP Bowden, D Tabor. The Friction and Lubrication of Solids. Oxford, UK: Clarendon Press, 1958.

70. M Casey, J Wilks. The friction of diamond sliding on polished cube faces of diamond. J Phys D (Appl Phys) 6, 15:1772-1781, 1973.

71. IP Hayward. Friction and wear properties of diamond and diamond coatings. Surf Coat Technol 49:554-559, 1991.

72. IP Hayward, IL Singer. Tribological behaviour of diamond coatings. In: Second International Conference on New Diamond Science and Technology. Pittsburgh, PA: Materials Research Society, 1991, pp. 785-789.

73. IP Hayward, IL Singer, LE Seitzman. Effect of roughness on the friction of diamond on CVD diamond coatings. Wear 157:215-227, 1992.

74. D Tabor. Adhesion and Friction. In: J.E. Field, ed. The Properties of Diamond. New York: Academic Press, 1979, pp. 325-350.

75. D Crompton, W Hirst, M.G.W. Howse. The wear of diamond. Proc R Soc London Ser A 333-1595:435-454, 1973.

76. Z Feng, Y Tzeng, JE Field. Friction of diamond on diamond in ultra-high-vacuum and low-pressure environments. J Phys D (Appl Phys) 25, 10:1418-1424, 1992.

77. FP Bowden, AE Hanwell. The friction of clean crystal surfaces. Proc R Soc (London) Ser A 295:233-243, 1966.

78. MT Dugger, DE Peebles, LE Pope. Counterface material and ambient : role in the tribological performance of diamond films. In: YW Chung, AM Homola, GB Street, eds. Surface Science Investigations in Tribology: Experimental Approaches. ACS Symposium Series 485, Washington, DC: American Chemical Society, 1992, pp. 72-102. 


\section{REPORT DOCUMENTATION PAGE}

Public reporting burden for this collection of information is estimated to average 1 hour per response, including the time for reviewing instructions, searching existing data sources gathering and maintaining the data needed, and completing and reviewing the collection of information. Send comments regarding this burden estimate or any other aspect of this collection of information, including suggestions for reducing this burden, to Washington Headquarters Services, Directorate for Intormation Operations and Reports, 1215 Jefferson Davis Highway, Suite 1204. Arlington. VA 22202-4302, and to the Office of Management and Budget, Paperwork Reduction Project (0704-0188), Washington, DC 20503.

\section{\begin{tabular}{l|l|l}
\hline 1. AGENCY USE ONLY (Leave blank) & 2. REPORT DATE & 3. REPORT TYPE AND DATES COVERED
\end{tabular}}

\begin{tabular}{|l|l} 
July 2002 & Technical Memorandum \\
\hline
\end{tabular}

4. TITLE AND SUBTITLE

Surface Characterization Techniques: An Overview

6. AUTHOR(S)

Kazuhisa Miyoshi

7. PERFORMING ORGANIZATION NAME(S) AND ADDRESS(ES)

National Aeronautics and Space Administration

John H. Glenn Research Center at Lewis Field

Cleveland, Ohio 44135-3191

9. SPONSORING/MONITORING AGENCY NAME(S) AND ADDRESS(ES)

National Aeronautics and Space Administration

Washington, DC 20546-0001

\section{SUPPLEMENTARY NOTES}

Responsible person, Kazuhisa Miyoshi, organization code 5160, 216-433-6078.

12a. DISTRIBUTION/AVAILABILITY STATEMENT 12b. DISTRIBUTION CODE

Unclassified - Unlimited

Subject Category: 23

Distribution: Nonstandard

Available electronically at http:/gltrs.grc.nasa.gov/GLTRS

This publication is available from the NASA Center for AeroSpace Information, 301-621-0390.

13. ABSTRACT (Maximum 200 words)

To understand the benefits that surface modifications provide, and ultimately to devise better ones, it is necessary to study the physical, mechanical, and chemical changes they cause. This chapter surveys classical and leading-edge developments in surface structure and property characterization methodologies. The primary emphases are on the use of these techniques as they relate to surface modifications, thin films and coatings, and tribological engineering surfaces and on the implications rather than the instrumentation.

\section{SUBJECT TERMS}

Solid surfaces; Surface roughness; Surface properties OF REPORT

Unclassified
17. SECURITY CLASSIFICATION
18. SECURITY CLASSIFICATION OF THIS PAGE

Unclassified
19. SECURITY CLASSIFICATION OF ABSTRACT

Unclassified
8. PERFORMING ORGANIZATION

REPORT NUMBER

E-12968
10. SPONSORING/MONITORING AGENCY REPORT NUMBER

NASA TM-2002-211497 